AlllOb 978324

of Commerce

National Bureau

of Standards

NBS

PUBLICATIONS

\section{Computer Science and Technology}

NBS Special Publication 500-101

Care and Handling of

Computer Magnetic

Storage Media
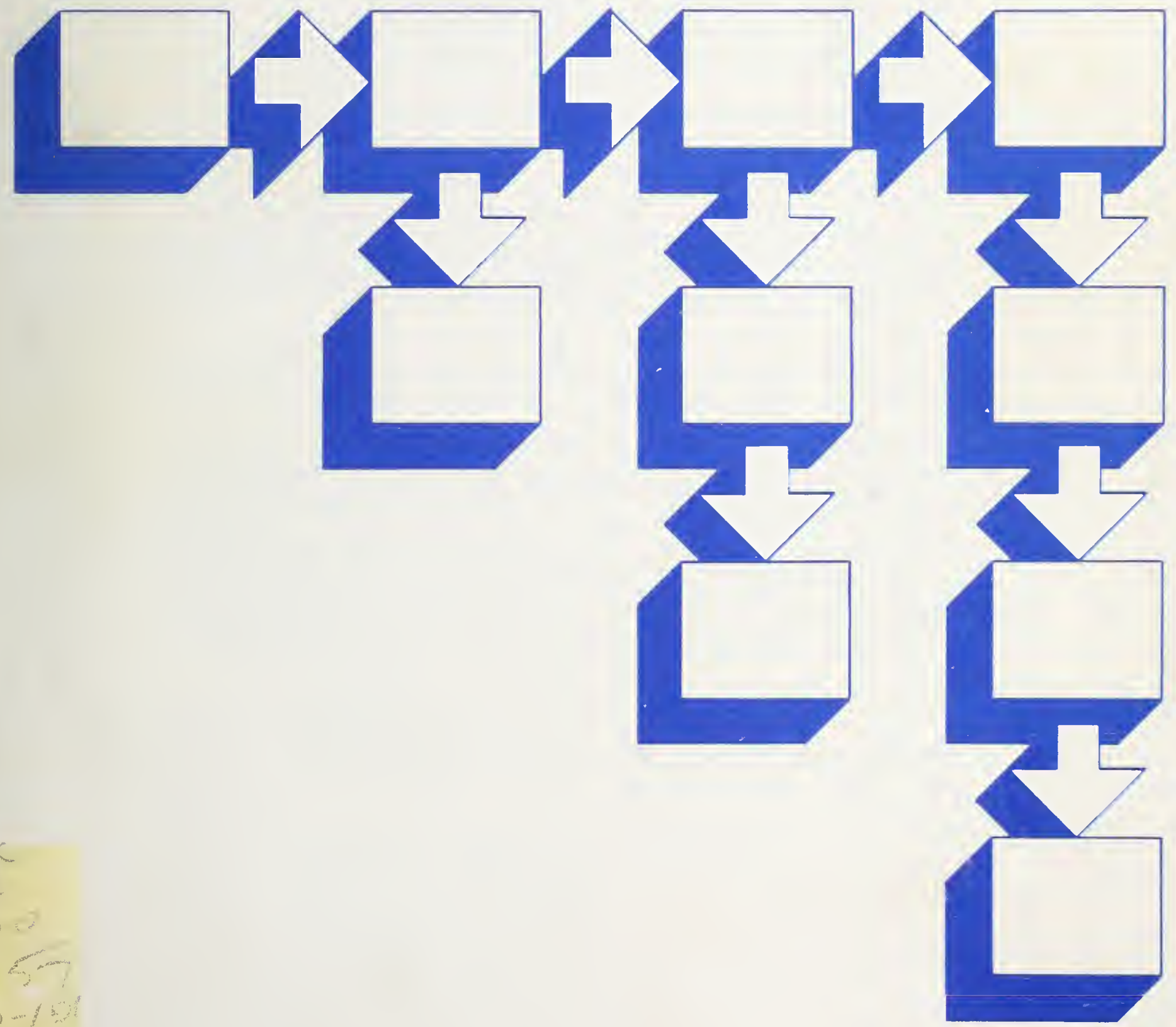


\section{NATIONAL BUREAU OF STANDARDS}

The National Bureau of Standards' was established by an act of Congress on March 3, 1901. The Bureau's overall goal is to strengthen and advance the Nation's science and technology and facilitate their effective application for public benelit. To this end, the Bureau conducts research and provides: (1) a basis for the Nation's physical measurement system, (2) scientific and technological services for industry and government, (3) a technical basis for equity in trade, and (4) technical services to promote public safety. The Bureau's technical work is performed by the National Measurement Laboratory, the National Engineering Laboratory, and the Institute for Computer Sciences and Technology.

THE NATIONAL MEASUREMENT LABORATORY provides the national system of physical and chemical and materials measurement; coordinates the system with measurement systems of other nations and furnishes essential services leading to accurate and uniform physical and chemical measurement throughout the Nation's scientific community, industry, and commerce; conducts materials research leading to improved methods of measurement, standards, and data on the properties of materials needed by industry, commerce, educational institutions, and Government; provides advisory and research services to other Government agencies; develops, produces, and distributes Standard Reference Materials; and provides calibration services. The Laboratory consists of the following centers:

\section{Absolute Physical Quantities ${ }^{2}$ - Radiation Research - Chemical Physics - Analytical Chemistry - Materials Science}

THE NATIONAL ENGINEERING LABORATORY provides technology and technical services to the public and private sectors to address national needs and to solve national problems; conducts research in engineering and applied science in support of these efforts; builds and maintains competence in the necessary disciplines required to carry out this research and technical service; develops engineering data and measurement capabilities; provides engineering measurement traceability services; develops test methods and proposes engineering standards and code changes; develops and proposes new engineering practices; and develops and improves mechanisms to transfer results of its research to the ultimate user. The Laboratory consists of the following centers:

Applied Mathematics - Electronics and Electrical Engineering ${ }^{2}$ - Manufacturing Engineering - Building Technology - Fire Research - Chemical Engineering ${ }^{2}$

THE INSTITUTE FOR COMPUTER SCIENCES AND TECHNOLOGY conducts research and provides scientific and technical services to aid Federal agencies in the selection, acquisition, application, and use of computer technology to improve effectiveness and economy in Government operations in accordance with Public Law 89-306 (40 U.S.C. 759), relevant Executive Orders, and other directives; carries out this mission by managing the Federal Information Processing Standards Program, developing Federal ADP standards guidelines, and managing Federal participation in ADP voluntary standardization activities; provides scientific and technological advisory services and assistance to Federal agencies; and provides the technical foundation for computer-related policies of the Federal Government. The Institute consists of the following centers:

Programming Science and Technology - Computer Systems Engineering.

'Headquarters and Laboratories at Gaithersburg, MD, uniess otherwise noted;

mailing address Washington, DC 20234.

${ }^{2}$ Some divisions within the center are located at Boulder, CO 80303. 


\section{Computer Science

NBS Special Publication 500-101

\section{Care and Handling of Computer Magnetic Storage Media}

Sidney B. Geller

Institute for Computer Sciences and Technology National Bureau of Standards

Washington, DC 20234

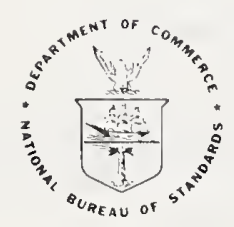

U.S. DEPARTMENT OF COMMERCE Malcolm Saldrige, Secretary

National Bureau of Standards

Ernest Ambler, Director

Issued June 1983 


\section{Reports on Computer Science and Technology}

The National Bureau of Standards has a special responsibility within the Federal Government for computer science and technology activities. The programs of the NBS Institute for Computer Sciences and Technology are designed to provide ADP standards, guidelines, and technical advisory services to improve the effectiveness of computer utilization in the Federal sector, and to perform appropriate research and development efforts as foundation for such activities and programs. This publication series will report these NBS efforts to the Federal computer community as well as to interested specialists in the academic and private sectors. Those wishing to receive notices of publications in this series should complete and return the form at the end of this publication.

\section{Library of Congress Catalog Card Number: 83-600536}

\section{National Bureau of Standards Special Publication 500-101}

Natl. Bur. Stand. (U.S.), Spec. Publ. 500-101, 135 pages (June 1983) CODEN: XNBSAV

\section{U.S. GOVERNMENT PRINTING OFFICE WASHINGTON: 1983}

\footnotetext{
For sale by the Superintendent of Documents, U.S. Government Printing Olfice. Washington. DC 20402 Price $\$ 5.50$ 


\section{PREFACE}

Computer magnetic tape is the prevalent mass storage medium used by industry and Government data processing organizations for both direct and archival data storage applications. A forecast for the storage media installed base in the Federal Government predicts that the Government inventory will consist of 11 million reels of magnetic computer tape by 1985 [1].

The improper care and handling of these computer magnetic tapes and other magnetic media such as flexible disk cartridges, is the major cause for serious and costly media and data losses by all users. This Special Publication has been written in response to this important problem. It presents a comprehensive set of care and handling guidelines and recommendations whose purpose is to assure the successful retrieval of the data which are stored on these media. It considers data retrieval in active areas such as data processing centers and tape libraries, as well as in long-term tape storage environments.

This Special Publication addresses the information needs of the ADP installation managers, the operating personnel and the technical staff.

The author gratefully acknowledges the contributions of Candice E. Leatherman to the production of the Special Publication. Her dedication, patience, secretarial skills, and suggestions were indispensable to the writing of this document. 

1. Computer Magnetic Media Care and Handling

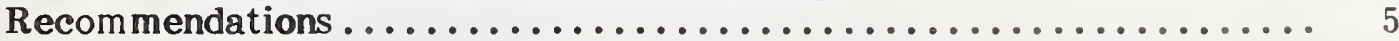

1.1 Descriptions of Recommendations in Section $1 \ldots \ldots \ldots \ldots \ldots \ldots$

1.2 Computer Tape Physical Handling Recommendations ........... 9

1.3 Clean Handling Recommendations ..................... 19

1.4 Environmental Recommendations .................... 24

1.5 Transportation Recommendations ................... 26

1.6 Effects of Extreme Environments on Computer

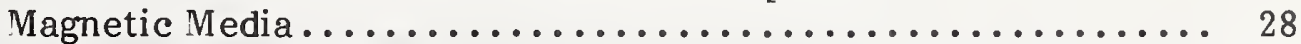

1.7 Fire Recommendations $\ldots \ldots \ldots \ldots \ldots \ldots \ldots \ldots \ldots \ldots \ldots \ldots \ldots \ldots \ldots$

1.8 Water Recommendations .......................... 34

1.9 Effects of Magnetic Fields on Recorded Magnetic Media ....... 37

1.10 Exposure to Fields and Devices Recommendations ........... 41

1.11 Questions and Answers Relative to the Effects of

Magnetic Fields on Recorded Media ................. 45

1.12 Magnetic Field Recommendations .................... 49

1.13 Scheduled Computer Tape Maintenance ................. 52

1.14 Scheduled Computer Tape Maintenance Recommendations ...... 53

1.15 Flexible Disk Cartridges ......................... 57

1.16 Flexible Disk Care and Handling Recommendations .......... 58

1.17 Computer Tape Transport Care .................... 62

2. Long-Term Storage - Care and Handling ................... 64

2.1 Long-Term Archival Storage - Large Population Cases ......... 66

2.1.1 Preparation Phase......................... 6 ?

(1) The Quality of the Tapes that are Chosen for

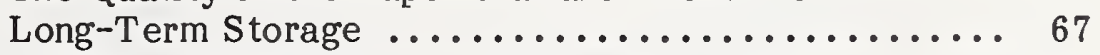

(2) Physical Preparation of the Computer Tapes

Prior to Long-Term Storage ................ 70

(3) Recommendations for Computer Tape Physical

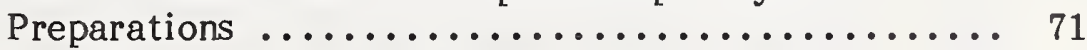

(4) Preparation of the Data Prior to Long-Term

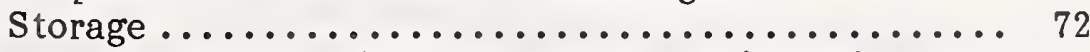

(5) Recommendations for the Preparation of Archival

Data .............................. 73

2.1 .2 Storage Phase $\ldots \ldots \ldots \ldots \ldots \ldots \ldots \ldots \ldots \ldots \ldots \ldots \ldots$

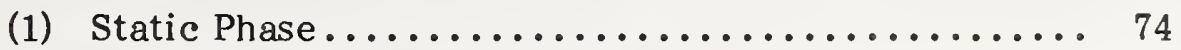

(2) Recommendations (Static Phase) ............. 75

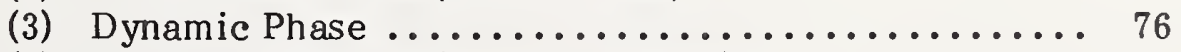

(4) Recommendations (Dynamic Phase)............ 77 
2.1.3 Reactivation Phase....................... 78

(1) Recommendations for the Reactivation Phase...... 79

2.2 Long-Term Archival Storage - S mall Sample Population Case .... 80

2.2.1 Background Theory for Small Sample Population

Recommendations......................... 81

2.2.2 Preparation Phase....................... 84

(1) Quality of Tapes that are Chosen............. 84

(2) Physical Preparation Prior to Long-Term Storage............................ 85

2.2 .3 Storage Phase $\ldots \ldots \ldots \ldots \ldots \ldots \ldots \ldots \ldots \ldots \ldots \ldots \ldots$

(1) Static Recommendations ................. 86

(2) Dynamic Recommendations ............... 86

2.2.4 Reactivation Phase Recommendations ............ 86

3. Scheduled Maintenance of Computer Magnetic Tapes ............ 88

3.1 Computer Tape Errors ....................... 88

3.1.1 Scheduled Maintenance Cost Factors ............ 89

3.2 Types of Computer Magnetic Tape Maintenance Equipment ..... 91

3.2.1 Tape Cleaners ........................... 91

3.2 .2 Tape Certifiers ......................... 92

3.2.3 Tape Evaluators/Testers .................... 93

3.2.4 Miscellaneous Devices ...................... 94

3.3 Tape Rehabilitation $\ldots \ldots \ldots \ldots \ldots \ldots \ldots \ldots \ldots \ldots \ldots . \ldots \ldots$

3.3.1 Class of Work .......................... 95

3.3.2 Product Requirements ....................... 95

3.3.3 Record Keeping........................... 96

3.3.4 Quality Assurance Requirements ................ 98

4. Tape Management Systems (TMS) $\ldots \ldots \ldots \ldots \ldots \ldots \ldots \ldots \ldots \ldots . \ldots . \ldots 9$

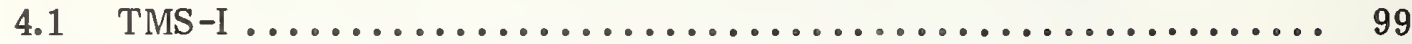

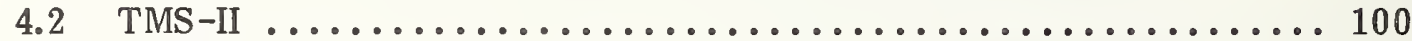

4.3 TMS-III .................................. 102

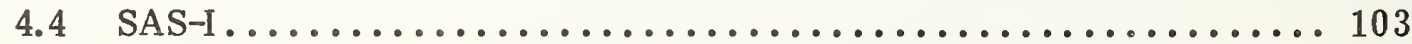


5. Clean Rooms and Clean Room Operations Guidelines ............. 104

5.1 Description of Clean Rooms ..................... 104

5.2 Various Media Clean Rooms in Industry and

5.3 General Guidelines for all Types of Clean Rooms

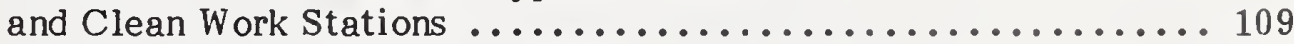

5.4 Guidelines for Nonlaminar Airflow Clean Rooms

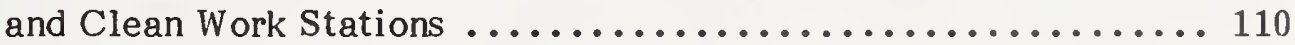

5.5 Guidelines for Laminar Airflow Clean Rooms and Clean Work Stations

6. References.................................... 112

7. Appendix A. Handling and Environmental Effects ............. 115

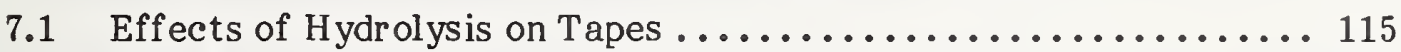

7.2 Effects of Humidity on Tape Abrasivity ................. 116

7.3 Effects of Viscoelasticity on the Tape Pack............... 117

7.4 Effects of Temperature, Hum idity, and Winding

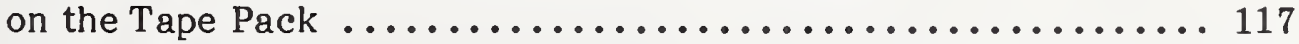

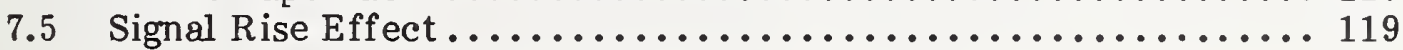

8. Appendix B, Computer Magnetic Tape Recording

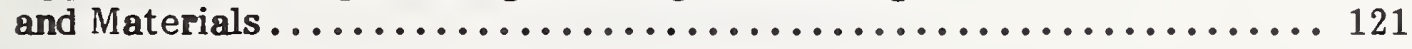

8.1 Computer Magnetic Tape Recording .................. 121

8.2 Magnetic Media Particles ........................ 124

9. List of Symbols and Abbreviations ..................... 127

\section{LIST OF TABLES}

Table 1. Candidate Cleaning Solvents...................... 20

Table 2. Computer Tape Tests, Measurements, and Exam inations ....... 68

Table 3. General Examination of Tapes .................... 69

Table 4. Maximum Dropouts/Errors per Reel .................. 97

\section{LIST OF FIGURES}

Figure 1. Effects of Permanent Magnetic Fields on Recorded Tapes ...... 39

Figure 2. Effects of Varying Magnetic Fields on Recorded Tapes ........ 40

Figure 3. Effects of Temperature and Humidity on the Hydrolysis of Tape Binders 


\title{
CARE AND HANDLING OF \\ COMPUTER MAGNETIC STORAGE MEDIA
}

\author{
Sidney B. Geller
}

\begin{abstract}
This Special Publication deals with the physical/chemical preservation of computer magnetic storage media--principally computer magnetic tapes--and their stored data, through the application of proper care and handling methods under various conditions. It emphasizes the media handling methods and environmental conditions which should be instituted during the course of controllable day-to-day and long-term archival storage activities. It also considers measures which can be initiated during media transit and in the aftermath of catastrophic or uncontrollable events. However, the publication does not address the problem of computer storage system security from the viewpoint of data theft or intentional data modification.
\end{abstract}

Key words: Archival storage; care and handling; certification/evaluation; computer tape, magnetic; clean rooms; environmental effects; flexible disks; magnetic fields, effects of; rehabilitation, computer tape; scheduled maintenance, computer tape; storage media, computer magnetic; tape management systems.

\section{INTRODUCTION}

Total computer installation security can be achieved only with a full backup system including hardware, software, and all storage media. Unfortunately the duplication of a complete computer system at alternate sites is prohibitively expensive (and unnecessary) for most organizations. In many instances, the central processor, the communications equipment, and the peripheral devices can be replaced in the wake of a catastrophic event. In fact, a security arrangement may consist of an agreement for emergency backup operations with another organization's system. However, the loss of, or the destruction of, vital data files and programs stored on computer magnetic media such as tapes can result in extremely serious and costly recovery problems, if indeed such recovery is at all possible. Therefore, the maintenance of a secure media storage system is absolutely necessary and must be given a high organizational priority $[2],[3] .1$

Typically, most organizations maintain their computer tape libraries in close proximity to their data processing facility in order to achieve efficient and rapid access to their tapes with minimum temperature-humidity variations in transit. In addition to the media stored in the tape library, duplicates of the critical tapes (and disks) are often stored in off-site locations including commercial vaults. ${ }^{2}$ In instances where increased security is desired for on-site media storage, special heat, fire, and humidity resistant vaults and safes are employed. In some organizations, large environmentally controlled, on-site walk-in vaults are

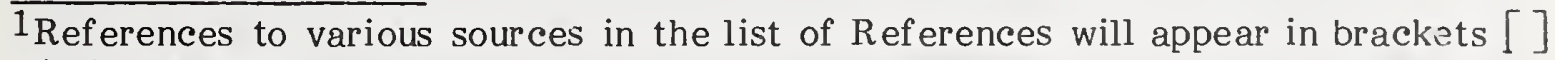
within the text.

${ }^{2}$ Commercial storage facilities for computer magnetic media can of ten be located through the Yellow pages of the telephone directory (see sec. 1.2-(33)).
} 
constructed which are both fire and intrusion resistant. The formal control of personnel access to all of these locations is another important security factor which should be considered in the initial design phase.

This Special Publication outlines recommendations for maintaining the security and quality of computer magnetic media and the stored data in the following locations:

- Active environments (ADP operational areas, testing and research areas).

- Storage environments (libraries and vaults; long-term archival storage).

- In transit.

There are variations in the care and handling methods for each area. For example, media whose lifetime will consist of transits from an active tape library to the operational data processing facility and return, are handled and selected differently from media held in long-term archives. Master or vital tapes which are in transit through uncontrolled environments are of ten packaged in specialized containers which shield these media from the environment.

This Special Publication considers the care and handling activities for computer storage media in the following categories:

(a) Computer storage media management which is based upon recommended methods for the controllable, physical care and handling of the media and their operating and storage environments. This category constitutes the major portion of this publication (see sec. 1.). It considers:

- The proper physical and clean handling of the storage media by the personnel in the test laboratory, the tape library, the long-term archives, and the computer installation. It outlines activities which should either be initiated or avoided by the operating personnel in order to minimize the physical/chemical deterioration of the media and to maximize their potential for data recovery.

- Environmental requirements which include the conditions under which the medium is tested, stored, and operated as well as those under which the magnetic tape is transported. These conditions include specified temperatures and humidities, degrees of local cleanness, enclosures, as well as environments which are permeated by subtle energy types such as electromagnetic fields. In most instances, these environmental factors can be controlled through careful site planning, construction, and system maintenance.

- The recovery of damaged media and data. This activity is either a reflection of the failure to achieve the maximum potential of the preceding categories or the result of an uncontrollable system, environmental, or media breakdown.

(b) Controlled and protective management of computer tapes through the use of special computer sof tware programs such as Tape Management Systems (TMS) (see sec.4.). These management systems interact with the operation of the actual data processing systems whose storage media are being protected. They control the flow of the media through the system, 
perform error logging functions, and assist with the required tape maintenance scheduling.

(c) Scheduled maintenance of active and stored computer magnetic tapes (see sec. 3.). This maintenance includes the cleaning, evaluating (or certifying), rehabilitating, and exercising of the media according to specific criteria. These criteria can be tracked through the use of reports which are derived from Tape Management Systems (TMS), from less complex error-logging sof tware systems, or through manual check-off procedures.

(d) The long-term archival care and handling of computer magnetic tapes. This is another major area in this publication (see sec. 2.). A survey conducted for the National Archives and Records Service in 1975 indicated that Federal agencies had approximately 200,000 reels which contained information of long-term archival value [4]. A 1982 estimate projected an annual growth rate of $7 \%$ to $15 \%$ for magnetic tapes and a $25 \%$ to $40 \%$ annual growth rate for flexible disk cartridge media.

There are a number of criteria which should be met by a medium which is to be maintained in long-term archival storage. Among these are information density, data permanence, minimal maintenance, data costs, speed of access, machine independence, and media standardization. It was concluded that presently no available magnetic storage medium meets a sufficient number of these criteria [4]. However, since no alternate medium exists at this time, the effective care and handling of computer magnetic tape must be considered since this is the prevalent medium now used for long-term storage of summary and backup data. It is of interest to note that due to today's higher bit recording densities and lower computer tape prices, the cost per stored bit is less by approximately five orders of magnitude than the bit cost of 25 years ago.

Although only the archival care of computer magnetic tapes is discussed in this publication, any complete analysis of archival factors must also consider the preservation and security of the entire storage entity; this includes the lifetime of the system, the information contents, and the information codes [5].

The long-term archival care-and-handling methods for computer tapes which are described in section 2. stem either from: (a) results which were based on empirical observations derived from large, diverse tape sample populations, or from (b) analytically and experimentally based conclusions which were derived from small sample populations.

There are differences of opinion relative to the amount of formal scheduling which is required for archival tape maintenance. For example, some organizations [6] recommend extensive long-term archival computer tape care and handling programs which incorporate scheduled cleaning, exercising, and rechecking of most stored tapes, as well as the scheduled recopying of vital or master tapes. Alternately, one organization with a large tape archive has adopted an approach in which there is no formally scheduled archival tape maintenance program. Instead, the tapes are cleaned and reread only when data errors are encountered after they are recalled from archives [7]. The advent of improved tape binder formulations and smoother tape surfaces in recent years has probably reduced the risk inherent in this latter approach because surface smoothing techniques have produced tapes with higher bit 
density capabilities, less debris (leading to longer life), and less read/write head wear.

In addition, other organizations have instituted programs of scheduled archival care and handling of their computer tapes which is midway in complexity between the two preceding approaches. However, since there is no unified or analytic discipline available at present with which the care and handling effects on the long-term properties of computer magnetic tapes can be completely predicted, it is presently not possible to arrive at a final conclusion about a "best" method for ar chival care.

Appendix A discusses some of the underlying mechanisms which cause physical/chemical deterioration of the media and the subsequent data losses. These include:

(a) Extreme values and variations in environmental temperatures and humidities, and their resultant effects on coating hydrolysis, tape surface abrasivity, and tape pack pressures, and

(b) Improper tape winding processes and the resultant viscoelastic responses of the tape which lead to tape pack damage.

The care and handling information in this document has been derived from:

- Multiple sources in the open, non-proprietary literature including industry and Government publications and standards documents,

- Personal communications and meetings with media specialists in industry and Government who have been very informative and cooperative with the Institute for Computer Sciences and Technology (ICST) personnel over the years. There is no divulgence of proprietary information in this publication.

- Extensive experience gained in the ICST, Computer Storage Media laboratory which has been engaged in experimental activities and in the measurement, calibration, and production of magnetic media standard reference materials (SRM's) for more than a decade. 


\section{Computer Magnetic Media Care and Handling Recommendations}

Magnetic computer tapes and flexible disks consist of magnetic oxide particles dispersed in a suitable polymeric binder material that has been uniformly coated over the surface of a flexible polyester, or equivalent base material. The prevalent base substrate material is typically made of mylar (polyethylene teraphthalate) which is a stable non-magnetic material. The polymeric binder coating material is typically a polyester urethane elastomer which contains the dispersion of gamma ferric oxide $\left(\gamma-\mathrm{Fe}_{2} \mathrm{O}_{3}\right)$ magnetic particles as well as numerous chemical constituents which give the final product its required characteristics such as improved particle dispersion, tape flexibility, conductivity, and relative sof tness which ensures intimate physical contact between the tape and the record/reproduce transducer.

The retention of the magnetically recorded data is performed solely by the oxide particles with the remaining constituents of the tape coating acting as a binder or carrier for these particles. Any embedding of debris into the media surface, or any distortion of the mylar base substrate material or the coating or both, or any breakdown or alteration of the chemical structure of the binder will result in a mispositioning of the media during operation, or a direct loss or misalignment of the coating or particles; this causes a loss of data contents. Studies have shown that other than those caused by unwanted magnetic fields, that there is almost no loss of data contents which does not stem from an alteration in some physical or chemical property of the polymeric media components. Fortunately, many conditions which cause data losses through the physical and chemical deterioration of the media can be controlled through the use of housekeeping techniques such as cleanliness controls, temperature and humidity controls, regular media maintenance schedules, and computer tape operator training. In addition, security measures can be initiated to prevent data losses due to magnetic fields.

Improper handling is a common cause for both debris contamination and physical damage to the reels and computer tapes. These damages inevitably lead to data losses and costly reconstruction procedures. Sections 1 . and 2. present methods leading to the proper care and handling of computer magnetic tapes and flexible disks and their associated systems for use by the managerial and operating personnel at a computer installation, tape library, or long-term archive.

Media system designs which simplify the required functions of the operating personnel can reduce the potential damage to the tapes. For example, the design of the reel mounting hub mechanism on some types of tape transports encourages the operators to set the tape reel onto the supply hub and snap it into position by striking the outer flanges of the reel briskly; this causes tape edge and/or reel flange damage. This damage can be circumvented through the use of a self-locking hub mechanism on the drive which does not require physical mounting force, or by a fully automatic self-loading tape cartridge system which places a truly effective barrier against the operators potential for inflicting media damage both through the use of excessive force and through finger contact contamination of the tape. Self-locking hubs and self-threading mechanisms which require very little finger-to-tape contact are now found on the newer high density tape drives. Operators often attempt to speed up their operations through the use of short cuts. For example, they will insert a missing write enable ring into the tape flange without first demounting the tape from the drive. This often leads to tape damage 
in the form of creases on the length of tape which has been extracted from the vacuum column.

Installations have reported that a considerable amount of paper printer dust has been found on computer tapes whose drives are located in the same general area as the printers. The effect of this paper dust on tapes and drives is exacerbated by drives whose windows have been left open intentionally by operators during tape operations. Data processing installation managers are responsible for many of these problems, particularly when they do not insist upon the maintenance of proper and clean working conditions and habits.

The following are the principal causes for media damage and data losses on computer magnetic tapes:

(1) Physical mishandling by operating personnel.

(2) Lack of proper training for media handling personnel.

(3) Poor on-site management.

(4) Failure to control temperature and/or humidity in operational or storage areas.

(5) Contamination, debris, and cumulative wear products in the tape pack caused by poor tape manufacturing control, operator mishandling, and by defective tape transport components.

(6) Misadjusted or misaligned transports or other tape winding equipment which cause improper tape tensioning and winding.

(7) Lack of environmental cleanliness control and failure to adhere to proper clean operating practices.

(8) Subjecting the recorded media to close-in high intensity magnetic fields.

(9) Failure to properly select and pretest media for use in long-term storage.

(10) Failure to properly prepare and to ship media in proper containers.

(11) Failure to initiate protective measures against catastrophic events such as fire and flood.

(12) Failure to provide a proper tape and system maintenance schedule.

(13) Failure to perform visual inspection of the tape, the tape reel flanges, and hubs prior to operation or storage.

(14) Recording the data on computer tapes using modulation methods which are very sensitive to media cold-flow distortion with time in storage. For example, storing tapes which have been recorded at $32 \mathrm{bpmm}$ (800 bpi) (NRZI) has been found to sustain a high rate of data loss with time (see sec. 2.1.1-(4) and references [8] and [9]).

In addition to magnetic computer tapes and flexible disk cartridges, the recommendations in section 1. are also applicable to other types of flexible magnetic media such as cassettes, cartridges, and in many areas for magnetic stripe plastic cards and rigid disks.

Note: The mention of specific sources and manufacturers is not to be construed as an endorsement by the National Bureau of Standards of these items over other available equivalent items. In most instances, the reader is directed to references $[2],[10],[11]$, and [12] which contain listings of specific manufacturing sources for many of the items under discussion. 


\subsection{Descriptions of Recommendations in Section $\mathbf{1}^{3}$}

\section{$\underline{\text { Section }}$}

1.2 Computer Tape Physical Handling Recommendations

1.3 Clean Handling Recommendations

1.4 Environmental Recomm endations

1.5 Transpor tation Recommendations

1.6 Effects of Extreme Environments on Computer Magnetic Media

1.7 Fire Recommendations

1.8 Water Recommendations

1.9 Effects of Magnetic Fields on Recorded Magnetic Media

1.10 Exposure to Fields and Devices Recommendations

1.11 Questions and Answers Relative to the Effects of Magnetic Fields on Recorded Media

\section{$\underline{\text { Description }}$}

Describes the controllable physical handling activities which should be initiated during normal operations.

Describes the proper clean operational modes which prevent media and system contamination, and data losses.

Outlines the accepted temperature-humidity environments for computer tape operating, storage, and test areas.

Outlines protective measures for computer tapes in transit through unknown environments.

Discusses the results of some experiments with computer magnetic media which were performed in hot and cold environments.

Outlines fire safety measures and some possible recovery methods for tapes which have been subjected to an extremely hot environment.

Outlines some water safety measures and some possible recovery methods for tapes which have been subjected to water inundation or immersion.

Discusses the results of experiments with magnetic fields and their potential for data erasure.

Outlines the effects of a number of devices such as airport metal detectors, and fields such as x-ray, radar, and high voltages on recorded magnetic media of various kinds.

Answers a series of questions which were posed by the Office of Management and Budget (OMB).

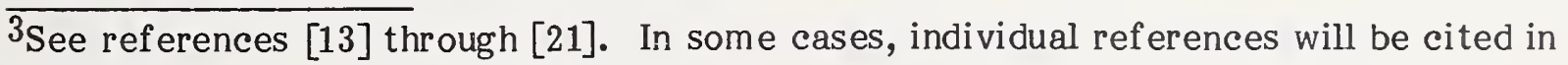
the text.
} 
$\underline{\text { Section }}$

1.12 Magnetic Field Recommendations

1.13 Scheduled Computer Tape Maintenance

1.14 Scheduled Computer Tape Maintenance Recommendations

1.15 Flexible Disk Cartridges and

1.16 Flexible Disk Care and Handling Recommendations

\subsection{Computer Tape Transport Care}

$\underline{\text { Description }}$

Outlines measures and materials for protecting computer magnetic media against data erasure by unwanted magnetic fields.

Discusses some problems which are encountered in setting up a scheduled tape maintenance program.

Presents a compilation of diverse recommendations for scheduling the cleaning, evaluation, and rehabilitation of computer magnetic tapes. It is offered as a guide to decisions by individual organizations.

Outlines care and handling procedures for flexible disk cartridges, including those in the office environment.

Presents some maintenance factors related to the read/write heads, the tape path, and tape dynamics.

Note: The original, customary units of measurement for all numeric values used in this publication are either U.S. engineering units or centimeter gram second electromagnetic units (cgs-emu). Numeric values in the International System of Units (SI) have been derived from these customary units. The SI units may have been rounded off and are therefore consistent with, but not exactly equal to, the customary units. Either the SI or customary units of measurement may be referred to, but the two measurement systems should not be intermixed or reconverted. Conversions from customary units to SI units have been performed according to IEEE Standard 268-1979, IEEE Standard Metric Practice. 
1.2 Computer Tape Physical Handling Recommendations

(1) Handle and lift a tape reel by its hub, with minimum flange contact or pressure; the hub is the strongest reel member.

(2) Never make direct contact with the tape through a window or aperture in the reel flange. When loading tape in a manual threading system, place a finger into the indentation in the raised molded disk on the side of the flange in order to rotate the reel.

(3) Never strike the reel flanges as a means of securing and mounting the tape onto a transport hub.

(4) Write enable rings should be removed from all recorded computer tapes which are placed into libraries or archives.

(5) Always completely demount a tape before replacing a missing write enable ring.
Remarks

(a) Tape canisters are designed to support the tape reel by its hub while wrap-arounds firmly support the tape reel by its flanges without sidewall pressure.

(b) Poor winding can produce irregularities in the tape pack 4 in the for $m$ of individually protruding tape layers. This places these layers in danger of physical (edge track) damage due to flange contact pressure during handling.

(c) Some installation managers have noted that tape edge damage is the prevalent cause for tape data losses followed by debris contamination. That is, careless handling and misadjusted drives are two principal causes for tape failure.

(a) There is a high potential for causing edge track damage through direct finger pressure on the tape (see sec. $1.2-(1)(b))$.

(b) Self-threading tape systems eliminate this potential form of tape damage.

(a) Apply most mounting pressure directly on the reel hub.

(b) A self-latching transport hub which requires minimum tape mounting pressure is a good means for reducing edge damage potential.

(a) This prevents the destruction of the recorded data by inadvertent overwriting.

(b) Some Tape Management Systems do not require this removal (see sec. 4.2).

(a) A length of tape which remains in a vacuum column can be creased if the tape is not completely rewound and removed from the transport before replacing its write enable ring.

\footnotetext{
4The tape pack is the cylinder which is formed by the tape after it is wound around the reel hub.
} 
1.2 Computer Tape Physical Handling Recommendations

(6) Make certain that the tape is correctly threaded before activating transport.

(7) Make certain that the tapes are mounted correctly and evenly on the transport hub before continuing with the loading operation.

(8) Close and secure transport doors and windows when the tape is in motion.

(9) Tape operators should be instructed to report signs of a sudden increase in transport debris formation (see sec. 1.17).

\section{Remarks}

(a) Improper threading can cause folding and creasing of the tape upon acceleration.

(a) Improper tape mounting can cause eccentric reel rotation which results in poor tape guidance, edge track damage, and tape creasing.

(a) Improperly closed (sealed) doors in the vicinity of transport vacuum columns can result in loading and running difficulties.

(b) Electrostatic fields produced by the running tape will at tract dust on to the tape surface through open transport windows (see sec. 1.10-(11)).

(a) Excessive debris can be caused by a misaligned, burred, scored, or def ective component in the tape path. This may result in abrasion damage to tape surf aces and edges as they are run over these components which include guides, capstans, heads, etc. Immediately report, locate, replace, or correct alignment of defective part.

(b) The onset of excessive debris will usually be accompanied by an increase in data error rates.

(c) The increase in debris may be caused by a tape with a faulty coating. Clean the entire tape path and try a known good tape. If the tape is faulty, recopy it immediately onto a new tape.

(d) See table 1. in section 1.3 which describes transport cleaning materials.

(a) Do a slow speed wind-rewind onto a new reel which has been checked for cleanliness and physical condition.

(b) Make certain that a new, proper tape label is affixed to the new reel.

(c) Read tape; particularly check edge tracks. Recopy tape if there is edge track damage (see secs. 1.2-(23) and 1.2-(24)). 
1.2 Computer Tape Physical Handling Recommendations

(11) Investigate and correct the causes for improperly wound tapes which appear when run on a particular transport. Stop the transport immediately if the tape pack is winding conically and is beginning to jam against a reel flange. Rewind the tape at once and check it for damage (see sec. 1.2-(21)).

\section{Remarks}

(a) Poor winding may be caused by misaligned or worn guides or machine winding tension is too low (see secs. $1.2-(7)$ and 1.17).

(b) This improper winding tension can result in both tape foldovers upon acceleration or deceleration (cinching), and in irregular tape edges which result in a rough tape pack.

(c) If the rough appearing pack has been caused by the original tape slitting process, replace it immediately. A poorly slit tape will usually produce debris at a high rate.

(a) After data recovery or recopying, either discard or cut the tape so as to remove the spliced region. Retain those lengths which are deemed to be applicable for continued use.

(b) Apply BOT-EOT ${ }^{5}$ reflective markers as required on the useful segments.

(c) Check and update the reel label.

(a) This also applies to self-loading tape car tridges which are being shipped or going into long-term storage. Make a notation on the reel that the grom met or strip must be removed before mounting the self-loading cartridge on the drive af ter storage or shipping.

(a) High speed rewind tends to produce uneven tensions and looser packs with a greater tendency for producing protruding layers. These looser packs are susceptible to edge damage and cinching under acceleration or deceleration. One causal mechanism is the lower layer-to-layer winding pressures which are caused by air entrapment during high speed winding. Tests have shown that interlayer trapped air in instrumentation tapes can be eliminated through the use of a packing roller which permits tight tape packing even at high winding speeds [22].

$\overline{5_{\text {See list of symbols and }}}$ abbreviations in section 9. 
1.2 Computer Tape Physical

Handling Recommendations

(15) Do not place any permanent magnet or other source of a magnetic field into direct contact with a recorded reel of magnetic tape $[13]$.

(16) Return all tapes to the library or archives as soon as possible after the completion of their run.

(17) Don't store or leave magnetic tapes mounted on tape transports which are turned off or are out of use.

(18) Never stack the computer tapes horizontally, always store them in a vertical (on edge) position. Never stand a tape vertically directly on its flanges; it must be enclosed in a canister or hung in a wrap-around.

(19) Each tape should be properly labeled either with an adhesive label or a record card holder in its front flange.

\section{Remarks}

(b) Slow speed rewind can also be performed on a tape cleaner with good wind-rewind and tensioning characteristics.

(a) See sections 1.11 and 1.12 for a detailed discussion of this problem. The effects of many different types of energy fields and equipments such as $\mathrm{x}$-ray, radar, nuclear, pressure, temperature, airport metal detectors, etc., are discussed in sections 1.9 and 1.10. Keep all permanent magnets at least $76 \mathrm{~mm}$ (3.0 in) away from recorded tapes.

(a) This enhances orderly bookkeeping procedures and diminishes the potential for the misplacement or the loss of tapes.

(b) This also minimizes the potential for adverse environmental effects on the media.

(a) This can cause tape misplacement or loss, and there is potential for damaging the tapes when the transport is reactivated.

(a) Horizontally stacked tapes can put pressure on the edge tracks of protruding layers.

(b) Use small mobile carts with vertical tape slot accommodations to both hold and transport tapes between the library and computer center.

(c) Horizontally stored tape packs can begin to separate if they were wound under a low tension.

(a) These labels should be applied before the write pass and should have the following (optional) type of information:

- Date of write pass.

- Serial and file number (also entered in a tape history log book)

- Recording density and mode.

- Current contents. 


\subsection{Computer Tape Physical Handling Recommendations}

(20) New tapes should be given a full length, slow speed wind-rewind pass before the first use.

(21) Each reel of tape should be inspected visually before use, particularly if it is being assigned to long-term archival service. Also inspect for the applicable conditions in section 3.3.2.

\section{Remarks}

- Current condition.

- Manufacturer identification.

(b) There are specialized labels which will assist with an organized tape cleaning and evaluation program. These labels, in addition to most of the items in (a) above, have boxes for checking of $f$ each time the tape is used and cleaned, date of first use, and disposition of tape af ter evaluation.

(c) Also see section 4. for labeling conditions when some Tape Management Systems (TMS) are used.

(a) Make certain that the transport is adjusted for proper winding tension.

(b) Alternately, it is recommended that this first pass be performed on a tape cleaner (see sec. 3.2.1).

Inspect the tape pack and reel for:

(a) Folded over tape layers: this is called cinching and can be caused by low or irregular winding tension regions in the tape pack which slip upon tape acceleration and deceleration.

(b) Curled or wavy irregular tape edges: this edge distortion or damage can be caused by a stretched tape edge or a long scratch along the edge.

(c) A non-circular tape pack shape: this is referred to as buckling or spoking. Buckling results in a non-sym metrical tape pack; spoking results in a polygon-shaped pack. Both shapes can be caused by a loose pack which is sometimes due to temperature induced hub contraction, or to low tape winding tensions. The hub contraction effect can be minimized by using hub material such as aluminum whose thermal coefficient of expansion is similar to that of the tape pack material itself (see sec. 7.4).

(d) Scattered tape windings with protruding tape layers: this can be caused by poorly slit tapes or by misadjusted or poorly designed tape transport mechanisms; it also results from high speed rewind operations. 
1.2 Computer Tape Physical Handling Recommendations

\section{Remarks}

(e) Cupped tape: this is a tape which is not flat transversely from edge-to-edge. Cupping can be caused by different hygroscopic and thermal coefficients of expansion between the coating and the base materials.

(f) An elevated bump or ridge on the outermost surface of the tape pack as observed bet ween the flanges of a vertically oriented reel of tape: this can be caused by a longitudinal scratch along a long length of the tape surf ace. The seratch is usually caused by a burr on a guide or head surface.

(g) Blocked tape: this is layer-to-layer adhesion which is caused by long ter $\mathrm{m}$ storage of the tape in an extreme temperature environment. Blocking can cause delamination or tape coating pull off. Blocking will manif est itself in the form of erratic tape motion and gross errors in the delaminated regions (see sec. 3.1).

(h) Loose debris: this may consist of coating particles or shreds of edge slittings. This is usualiy due to defective binders, poor manuf acturing control or inspection methods, and to defective or burred components in the tape path.

(i) Unusual tape discolorations caused by changes or deterioration in the binder chemistry.

(j) Cracked, chipped, bent, split, warped, or otherwise mechanically defective reels.

(k) Distortion defects such as cinching, spoking, and scattered windings without permanent damage, can sometimes be corrected by several full length, slow speed winding-rewinding cycles, followed by relaxation in the proper $\mathrm{T}-\mathrm{H}$ environment for approximately 24 hours. In more difficult cases, particularly for recorded tapes with valuable data, perform the suggest ed operations in section 1.2-(23). In the case of ridged, blocked, scratched, or discolored tapes: recorded tapes should be recopied if possible and unrecorded 


\subsection{Computer Tape Physical} Handling Recommendations

(22) Never place a recorded tape into long term storage which has not been given a full length, slow speed, wind-rewind pass prior to storage. Make sure that the drive is adjusted to produce proper winding tensions and smooth tape packs.
(23) Immediately check and recopy any recorded tape which appears physically or chemically damaged (i.e., creased, cinched, distorted edges, skewed, discolored, etc.).

\section{Remarks}

tapes should be discarded or stripped. 6 Contaminated tapes can be cleaned, rewound, and relaxed before using; if the tapes are new, contact the vendor and request replacement tapes.

(a) It has been found that tapes which have not been run over their full length (due to file positioning) will be prone to cinching when accelerated or decelerated after storage. This is caused by the tension differentials which develop in the wound tape pack due to the partial passes over the file areas.

(b) Normal winding tension prior to storage is generally given as $1.7 \mathrm{~N}$ (6 ozf) to $2.2 \mathrm{~N}$ (8 ozf). Excessive tension causes tape stretching and curvature while very low tension causes winding separation and tape folding (cinching). These deleterious effects become apparent on the initial runs after long-term storage.

(a) Although complete recopying may be difficult, it is mandatory to attempt to read the tape as soon as possible af ter the defect is noted.

(b) In difficult data recovery cases in which backup tape data does not exist, the following may be attempted:

(1) Relax the tape for 24 hours in the operating environment after winding and rewinding it over its full length several times at slow speeds.

(2) Try to recopy the data onto new reels. If unsuccessful:

- Try adjusting the system electronics to improve the reproduced signal levels (readjust back to normal after completion).

- Try to read on different computer tape transports.

- In some cases, balky tapes can be read successfully in reverse.

6Stripping is the process of excising the defective portion of a tape (usually the initial footage). 
1.2 Computer Tape Physical

Handling Recommendations

(24) As a rule, avoid the application of external pressure against the physical tape-head-interface as a means for recovering data through improved head-to-tape contact.

(25) Degauss (i.e., demagnetize) the $\mathrm{read} / \mathrm{write}$ heads and other magnetizable metal components such as rollers, capstans, and guides on a schedul ed basis.

(26) Initiate an operator training program.7

(27) Make certain that there is at least one person on-site who is responsible for the activities of the operators and for adherence to all of the recommended principles in section 1.

\section{Remarks}

- If tape curvature is causing read skew error, try 10-15 full straightening passes on the tape drive to reduce curvature before reading again.

(3) If the data is sufficiently valuable, try the following item (24) only over the defective portion of the tape as it is being read. This may be useful for a cupped, cinched, or edge distorted tape.

(a) Excessive external pressure increases tape and head wear as well as the production of debris. However, this process may be attempted sparingly if the data is deemed to be of sufficient value. Application of this pressure when the tape is in a constant retry mode over a small area can result in the serious shedding of tape coating.

(b) A clean, sof t, non-shedding material wrapped on a slender rod is recommended, rather than direct finger pressure which may produce unnecessary contaminants in the system.

(a) See Magnetic Field Recom mendations section 1.12-(5).

(a) Extracts from this and other referenced publications may be a useful source of training inf ormation.

(a) This individual should be technically oriented and should have the backing of upper management for any personnel decisions that are made.

(b) This individual should be capable of analyzing the causes for media problems and initiating immediate

\footnotetext{
7 This includes training for computer tape librarians who also handle the media on a daily basis. There are computer librarian development workshops which offer appropriate courses for these personnel.
} 


\subsection{Computer Tape Physical Handling Recommendations}

(28) (Optional) Monitor the condition of the tape library or archive by installing control tapes into storage along with the regular tapes. This will assist with the detection of data and/or media deterioration which has been caused by the loss of system or media quality or environmental controls (see sec. 2.1.2-(3)).

(29) (Optional) Institute diagnostic programs such as peripheral test sequences.

(30) (Optional) Initiate a scheduled tape maintenance program.

(31) (Optional) Initiate error logging sof tware programs which provide an analysis of the tape errors.
(32) (Optional) Institute a Tape Management System (TMS).

\section{Remarks}

corrective action. This person should maintain a schedule for inspections, cleaning, repair, and replacement of system components as required.

(a) These control tapes should be recorded with a test signal at the same density and on the same system as the stored tapes. They should then be inspected on a scheduled basis for physical condition and data errors.

(b) The number and type of control tapes are based on:

- The size and the importance of the files and records.

- The number of stored tapes emanating from individual manuf acturing sources.

- The known history of particular tapes in storage.

Note: It is suggested by some sources that testing a $3 \%$ random sample drawn from a large file is a good statistical indicator of the condition of the total file. (See sec. $2.1 .2-(4)(d)$.

(a) These programs assist the technical personnel with a means for determining the source of media problems in the computer system.

(a) See section 3.

(a) In lieu of off-line evaluation of tapes (see sec. 3.2.3), these error logging sof tware programs permit tapes to be evaluated on the actual system prior to or during their application. For example, one such program gives (see sec. 4.4):

- Location of tape errors.

- Length of tape errors.

- Total retries per tape.

- Which tape should be cleaned, discarded, or stripped.

(a) These sof tware systems encompass numerous functions beyond those in item (31) above (see sec. 4.). 


\subsection{Computer Tape Physical}

Handling Recommendations

(33) (Optional) If necessary, use a commercial tape repository for storing important backup tapes.

\section{Remarks}

(a) Before using any repository, request the following information:

- The temperature and humidity ranges which are maintained in the storage areas (see sec. 1.4).

- The level of cleanness and how it is achieved and maintained (see secs. 1.3 and 5.$)$.

- Is there positive air pressure?

- Are there precipitrons?

- The fire, water, and magnetic field security measures (see secs. 1.7, 1.8, and 1.12).

- Are there individual tape vaults or safes available for additional security within the repository (see secs. 1.7 and 1.12)?

- The accessibility of the stored tapes by unauthorized personnel, including other subscribers to repository space. Does each subscriber have separately secure areas?

- What are the security screening measures for persons entering the repository area?

- Are the stored tapes accessible upon demand?

- Is there a 24 hour guard system?

Electronic, human, or both? 
1.3 Clean Handling Recommendations ${ }^{8}$

(1) Minimize finger contact with computer magnetic tapes.

(2) Keep hands clean in order to reduce normal skin oils. The use of lint-free (nylon gloves) is highly recom mended.

(3) Never allow the end of the tape to touch an unclean surface such as the floor.

(4) Never smoke, eat, or drink in the computer room or the tape library.

(5) Do not bring dirty or dust covered objects or equipment into the computer room or tape library.

(6) Do not remove the computer tape from its canister or wrap-around until it is ready to be mounted on the transport. Do not use wrap arounds with reels that have open apertures (windows) in their flanges particularly for long-term storage.

\section{Remarks}

(a) Fingers are a source of tape contamination. Fingerprints on either surface of the tape will eventually cause contamination of both sides.

(a) Oils will trap dust and other environmental contaminants such as lint onto the tape surface; these eventually produce tape errors (see sec. 3.1).

(a) This will contaminate the tape and spread the dirt onto transport guide components and the read/write heads.

(a) Interestingly, a speck of dust on the tape which is only $12.5 \mu \mathrm{m}(500 \mu \mathrm{in})$ in diameter can cause a $70 \%$ loss of signal level at $32 \mathrm{bpmm}$ ( $800 \mathrm{bpi})$. Assigning a smoking area anywhere in a computer room containing tape drives is bad policy, since tape in motion produces electrostatic fields which will attract airborne smoke particles. The detrimental effects of eating and drinking in the computer room are self-evident.

(a) As a basic rule, do not perform any operation which produces or spreads debris in the vicinity of the tapes.

(a) The canister and wrap-around can be made to provide the cleanest environment that the tapes will experience at any time during their normal lifetime. Open windows or apertures in the flanges circumvent the effective dirt sealing effect of the wrap-arounds. Keep canisters closed after removing or replacing the tape.

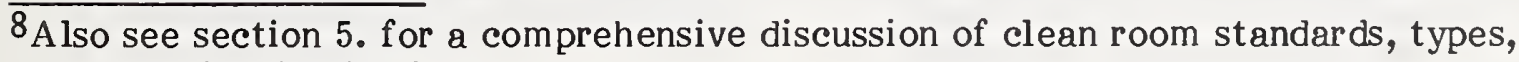
and operational guidelines. 
CLEANING SOLVENT

1. Freon TG

2. Xylene

3. Isopropyl Alcohol

4. Methyl Alcohol (Methanol)

\section{CHARACTERISTICS}

(a) Does not adversely affect polycarbonates, plastics, or neoprene.

(b) Cuts oil and grease.

(c) Has fastest rate of evaporation of the cleaners surveyed.

(d) Non-flammable, low toxicity.

(a) Damages polycarbonates and acrylics; doesn't harm neoprene.

(b) Cuts grease and oil very well.

(c) Slow evaporation rate.

(d) Extremely flammable and highly toxic.

(a) Doesn't harm polycarbonates, acrylics, or neoprene.

(b) Has limited capability to cut oil and grease.

(c) Slow evaporation rate.

(d) Flammable.

(a) Doesn't harm polycarbonates, acrylics, or neoprene.

(b) Limited capability to cut grease and oil.

(c) Fast evaporation rate.

(d) Flam mable.

NOTE: Solvents should not come in contact with the tape because, among other things, they may sof ten the coating and cause shedding. (Table courtesy reference [15].) 


\subsection{Clean Handling Recommendations}

(7) Always replace the computer tape into their canisters or wrap-arounds immediately after they are demounted from the transports. If possible, seal tapes in polyethylene bags particularly prior to shipping or long-term tape storage (see sec. 2.1.1-(3)).

(8) Always inspect empty reels, canisters, and wrap-arounds for cleanliness before using them with a computer tape; clean if necessary.

(9) Do not place or store computer tapes on the top of a transport since this exposes them to heat and dust from the blowers; this also affects the cooling of the unit. Also, don't store a tape in direct sunlight.

(10) Use reel identification labels with adhesive backings which do not leave a residue and can be removed easily. Never erase a label--replace it.

(11) Never use graphite pencils or water soluble felt tip pens on labels.

(12) Check transport for debris regularly. Clean tape transport components such as guides, vacuum chambers, capstans, and $\mathrm{read} / \mathrm{write}$ heads regularly and frequently; clean heads typically af ter each eight to ten tape mountings and the entire system once per shift. Clean with lint-free pads and permit parts to dry thoroughly before mounting the next tape.
Remarks

(a) Note that the polyethylene bags prevent the loss of coating lubricants through evaporation. This not only assures tape cleanliness but also provides for physical security of the tape reel.

(a) See section 1.3-(6).

(a) The effects of temperature and humidity on a reel of tape are discussed in section 7. Large variations in temperature can produce considerable changes in reel and hub pressure; this can result in serious tape pack damage. High humidity can cause coating deterioration due to hydrolysis. (See secs. 2.2.1 and 7.1.)

(a) See section 1.3-(5).

(a) Graphite pencils produce debris and the reel identification markings written with water soluble pens can be lost in case of water damage. In fact, avoid erasable marking devices.

(a) This reduces the spread of tape debris products and self-contamination. The read/write heads will both collect and act as reservoirs for tape debris which eventually will be permanently redeposited and fused into the tape surface (see sec. 3.1).

(b) Table 1. is a guide to transport cleaning chemicals. The cleaning of both equipment and tape should be a formal task which is specifically assigned and scheduled and not performed randomly (see sec. 1.2-(27)). 


\subsection{Clean Handling Recommendations}

(13) In operational facilities with with positive air pressure, always place the operating tape drives into high pressure locations, and debris producing equipment into low pressure areas.

(14) Unpack or store the minimum amount of required paper stock, cardboard, or other shedding materials in the computer room. Also, store these items in the low pressure area of the room.

(15) Store tapes which are waiting to be mounted or returned to the library or archives in the high pressure area of the computer room.

(16) Do not store tapes close to the floor.

(17) Wear lint-free smocks or overalls made of materials such as nylon.

(18) Do not wax floors or use steel wool pads on a buffer machine.

\section{Remarks}

Do not run tapes on equipment which have not been cleaned as indicated; clean immediately if excess debris is found (see sec. 1.17).

(a) Moving the printers, readers, card punches, and other debris sources downstream from the operating tape transports reduces tape contamination. It also places the drives upstream from any external dirt sources which may attempt to infiltrate the operating area.

(a) These items tend to shed debris (see sec. 1.3-(13)).

(a) Do this particularly if there are high levels of paper dust in the installation.

(b) Always store these tapes in a vertical position (see sec. 1.2-(18)). Horizontal positioning increases the contamination of the tape by airborne particles, particularly if there are open reel flange windows.

(a) See section 1.3-(5).

(a) It has been noted that personnel in computer installations which formally institute clean modes of operation will tend to perform higher quality work and display more pride in their job and organization (see sec. 5.).

(a) The wax is abraded by normal foot traffic and produces debris. Steel wool particles can cause considerable damage in a computer environment. 


\subsection{Clean Handling Recommendations}

(19) Exhaust vacuum cleaners into special wall receptacles or out of the computer area. Do not use dry mops, dusters, or brushes for cleaning. Damp mopping is recommended.

(20) The shelves, floors, equipment tops, and any other surfaces which are capable of holding dust should be cleaned periodically.

(21) Clean the dust off of the reel flanges before removing the wrap-arounds especially after long-term storage.

(22) Keep tape transport windows closed while operating.

(23) See section 5. for a listing of clean room standards and cleanliness guidelines for various types of clean rooms.

\section{Remarks}

(a) There are specialized, portable dual filtration vacuum cleaners which are claimed to permit the exhaustion of only the cleanest possible air within the computer room [12]. Filtration equipment in the air conditioning units also assists with airborne dust removal (see sec. 5.).

(a) This is a disadvantage of the wraparound as compared to the fully enclosed canister, although the narrower size of a wrap-around of fers a space advantage.

(a) Open windows permit dust to enter the tape path; this is particularly enhanced by electrostatic forces created by the moving tape (see sec. 1.10-(11)).

(a) These include:

- General guidelines for all types of clean rooms and clean work stations. See section 5.3.

- Guidelines for nonlaminar airflow clean rooms and clean work stations. See section 5.4 .

- Guidelines for laminar airflow clean rooms and clean work stations. See section 5.5 . 


\subsection{Environmental Recommendations}

(1) Maintain the operating, storage, and test areas for computer magnetic tapes at the following recommended temperatures, relative humidities, and maximum wet bulb temperatures. These conditions were tabulated from company specifications and national and international standards organization documents. The values in items (1)(a), (1)(b), and $(1)(c)$ appeared in the majority of sources:

(2) An al ternate experimentally

derived storage $\mathrm{T}-\mathrm{H}$

recommendation has been presented in the small sample case study for long-term storage as follows (see sec. 2.2.1):

(3) The maintenance of a constant $\mathrm{T}-\mathrm{H}$ environment within the given specification is extremely important. Temperature and humidity cycling cause large, deleterious stresses in the reel of tape.

\section{Remarks}

(a) Operating Areas (Recorded Tapes):

- Temperature range: $16^{\circ} \mathrm{C}-32^{\circ} \mathrm{C}$

$\left(60^{\circ} \mathrm{F}-90^{\circ} \mathrm{F}\right)$

- Relative Humidity Range: $20 \%-80 \%$

- Maximum Wet Bulb Temperature: $26^{\circ} \mathrm{C}$ (780F)

(b) Storage Areas (Recorded Tapes):

- Temperature range: $5^{\circ} \mathrm{C}-32^{\circ} \mathrm{C}$

(40F-90F)

. Relative Humidity Range: 20\%-80\%

- Maximum Wet Bulb Temperature: $26^{\circ} \mathrm{C}$ (780F)

(Unrecorded Tapes)

- Temperature range: $5^{\circ} \mathrm{C}-48{ }^{\circ} \mathrm{C}$ (400F-1200F)

- Relative Humidity Range: $20 \%-80 \%$

- Maximum Wet Bulb Temperature: $26^{\circ} \mathrm{C}$ (78 $\mathrm{F}$ )

(c) Test Areas:

- Temperature range: $21^{\circ} \mathrm{C}-25^{\circ} \mathrm{C}$ $\left(70^{\circ} \mathrm{F}-77^{\circ} \mathrm{F}\right)$

- Relative Humidity Range: $40 \%-60 \%$

Note: Test the tapes after a 24 hour conditioning period in the test facility.

(a) Storage Area (Recorded Tapes):

- Temperature range: $17^{\circ} \mathrm{C}-20^{\circ} \mathrm{C}$ $\left(62^{\circ} \mathrm{F}-68^{\circ} \mathrm{F}\right)$

- Relative Humidity Range: $35 \%-45 \%$

(b) Optimum Recommended Storage Vault

Values:

- Temperature: $18^{\circ} \mathrm{C}\left(65^{\circ} \mathrm{F}\right)$

- Relative Humidity: 40\%

Note: See sections 2.1 and 2.2 which deal with long term storage activities in detail.

(a) Damaging stresses in the tape pack can be produced by large $\mathrm{T}-\mathrm{H}$ variations even if they remain almost within the specified ranges. For example, tape problems can occur if stored tapes are subjected for a long period of time in an environment in which the temperature cycles between $12.8^{\circ} \mathrm{C}\left(55^{\circ} \mathrm{F}\right)$ and $35^{\circ} \mathrm{C}$ $\left(95^{\circ} \mathrm{F}\right)$ or the humidity cycles between $10 \%$ and $50 \%$ [6]. Excessive temperature and humidity conditions can also cause tape layer-to-layer adhesion (blocking) even if they are maintained constant. Extremely dry environments can cause 


\subsection{Environmental Recommendations}

(4) Do not produce heat or water vapor in the computer room operating area or tape library by using hot water heaters in these locations. Use an external area for these cooking purposes.

(5) The coatings on tapes which have been subjected to very high humidities can breakdown and become gum my due to hydrolysis.

\section{Remarks}

layer-to-layer adhesion due to static

buildup. Torn windings in the middle of a pack is a sure sign that the tape has been subjected to an extreme environment.

(a) This activity can affect the temperature and humidity in these areas. As previously noted, $\mathrm{T}-\mathrm{H}$ variations are a principal cause of tape problems.

(a) This coating deterioration can be reversed by storing the tapes in a cool and dry environment for an extended period of time (see secs. 2.2.1, 7.1, and fig. 3.). 


\subsection{Transportation Recommendations}

(1) The following are the recommended $\mathrm{T}-\mathrm{H}$ ranges for tapes during transit.

(2) There are a number of potential hazards which can be encountered during the transportation of computer tapes.

(3)

There are preventative measures which reduce the shipping hazards.9

\section{Remarks}

(a) Temperature range: $4{ }^{\circ} \mathrm{C}-32^{\circ} \mathrm{C}$ (400F-90\%F)

Relative humidity range: $20 \%-80 \%$

These hazards include:

(a) Dirt and water damage.

(b) Impact and vibration damage which can loosen the tape pack.

(c) Extreme and uncontrolled temperatures and humidities. These are difficult to control in transit. Procedures given in section 1.5-(4) should be followed upon receipt.

(d) Stray magnetic fields. Try to maintain a spacing of approximately $76 \mathrm{~mm}$ (3.0 in) between the tape reel and the sides of the shipping container.

These measures include:

(a) Proper final tape winding tensions bef ore shipping.

(b) The use of sealed, clean, water-tight, crushproof shipping containers lined with shock absorbent materials.

(c) Securing the free end of the tape (see sec. 1.2-(13)).

(d) Enclosing the canisters in polyethylene bags.

(e) Using proper container markings indicating both fragile media and vertical orientation.

(f) Using specialized tape carrying cases which supply all of the necessary safety features and which are designed for transportation by the individual traveler [12]. These light cases have rugged outer shells, internal foam padding, tight sealing against moisture and debris. However, extra care must be taken for vital tape records since these cases normally do not have the

9ne of the largest tape user agencies in the Federal Government (Social Security Administration) used a unique type of packaging for moving their extremely large tape library over a short distance from their original installation to a new one. They used hundreds of large portable coolers as packaging devices. These successfully provided the tapes with clean short-term physical protection, temperature and humidity control, and a waterproofed environment. Each cooler which held approximately 15 to 20 tapes, was reused many times during the move. 


\subsection{Transportation Recommendations}

(4) The following recommendations (a) through (g) give specific guidelines for the handling of tapes which have been transported between two different $\mathrm{T}-\mathrm{H}$ environm ents [16]:

\section{Remarks}

requisite interior to exterior tape spacing against magnetic fields. A ferromagnetic liner (shield) would be a valuable addition to this carrying case (see [10], secs. 1.12-(1) and 1.12-(2)).

(a) When a tape is moved from an external environment to a computer room environment, it should be acclimatized in its plastic canister for up to 24 hours.

(b) One hour of acclimatization should be allowed for each $\mathrm{OC}^{\mathrm{C}}$ and each $5 \%$ relative humidity $(\mathrm{RH})$ difference between the two environments.

(c) The temperature and humidity acclimatizations run concurrently.

(d) A void a temperature change of $10^{\circ} \mathrm{C}$ or more.

(e) Avoid tape condensation by avoiding temperature and humidity extremes.

(f) If a tape has been in an unknown external environment for up to eight hours, acclimatize the tape in the computer room environment for at least one hour longer than the known external time period.

(g) Tapes which have been in an unknown external environment for an unknown period of time should remain in their packing for 24 hours in the computer room environment. The tapes should then be unpacked and remain in their canisters for an additional eight hours in the computer room environment. Finally, give each transpor ted tape a full wind-rewind pass at the proper constant tension (typically $2.2 \mathrm{~N}$ (8 ozf)) before using. 


\subsection{Effects of Extreme Environments on Computer Magnetic Media}

Fire is one of the most destructive forces to which computer magnetic tapes can be subjected. Generally, direct contact with open flames or an extremely hot environment reduces the probability of data recovery to very close to zero. The direct damaging effects of heat are primarily in the form of physical/chemical media changes rather than magnetic data losses. Excessive heat affects the mechanical integrity of the flexible media and the reels through the loss of durability, warping, distortion, layer-to-layer adhesion (blocking), and binder breakdown and softening. In addition, the binder chemistry will be altered due to the volatile losses of binder components such as plasticizers.

Theoretically, gamma ferric oxide particles, which are the prevalent magnetic constituents used in most magnetic storage media, are capable of retaining their magnetization up to a temperature of approximately $675^{\circ} \mathrm{C}(1247 \mathrm{OF})$; this temperature is known as the Curie temperature. On the other hand, chromium dioxide particles, $\mathrm{CrO}_{2}$ used in some audio and video tapes, have a Curie temperature of approximately $135^{\circ} \mathrm{C}\left(275^{\circ} \mathrm{F}\right)$ and are therefore far more susceptible to a heat related data loss. Tests were performed in order to determine the effects of heat on the recorded magnetic information stored on tapes, cassettes, and plastic credit cards [13]. They were performed over a range of $38^{\circ} \mathrm{C}\left(100^{\circ} \mathrm{F}\right)$ to $182^{\circ} \mathrm{C}$ $\left(360^{\circ} \mathrm{F}\right)$. It was found that plastic digital cassette housings and credit cards began to distort physically at approximately $93^{\circ} \mathrm{C}\left(200^{\circ} \mathrm{F}\right)$ and could no longer be handled properly by their drives. Two reels of computer magnetic tapes ref used to load into the tape transport after they had been subjected to $110^{\circ} \mathrm{C}\left(230^{\circ} \mathrm{F}\right)$. The only apparent physical change at this time was a loss of tape stiffness. At first, this appeared to be a permanent condition since these tapes had not recovered after six weeks in storage. However, af ter two months, they were able to be run again; although the data could be recovered, the oxide appeared to be shedding badly. In such cases, the data should be recopied as soon as possible onto new tapes and the shedding tapes discarded.

Every heat test which was performed was terminated due to the physical failure of the media materials and housings rather than the loss of magnetic data. In one test, a recorded plastic credit card was constrained from warping by aluminum plates in order to isolate the physical effects of heat from its magnetic effects. There was no data loss observed after the card had been heated to $182^{\circ} \mathrm{C}\left(360^{\circ} \mathrm{F}\right)$ which was the temperature limit of the experimental oven.

Theoretically, the magnetic oxide particles in the media will not lose any of their magnetization at temperatures which are well below their Curie temperature. However, the tapes may become stiff and moisture may condense upon them after being subjected to very low temperatures. Recorded computer tapes which were stored at $-51^{\circ} \mathrm{C}\left(-60^{\circ} \mathrm{F}\right)$ for 24 hours could be read without loss af ter being relaxed (by careful, slow unwinding and rewinding) in the normal laboratory environment for one day.

Generally, tapes which have been subjected to extremely low temperatures for long periods of time should be relaxed and dried if necessary for a number of days at gradually increasing temperatures in order to relieve the stresses which developed with time. The shrinking of the tape due to exposure to extreme cold can produce 
layer-to-layer adhesion; this can cause tape tearing and surface coating damage in portions of the reel.

If the tapes have been subjected to inundation by, or immersion in water, there is a high probability of data recovery so long as a high temperature environment did not exist concurrently. The production of steam or very high humidity in the tape pack can produce extreme pack pressures leading to significant tape damage. The hygroscopic effects of the extreme humidity can also produce binder breakdown (see secs. 2.2.1 and 7.1). 


\subsection{Fire Recommendations}

(1) Avoid the storage of combustible materials in a tape storage area.

(2) Avoid the use of open flame producers, such as matches and lighters in a tape storage area. Smoking should be prohibited in this area.

(3) Store vital magnetic tapes in tightly closed metal or plastic containers.

(4) Areas in which computer tapes are stored should al ways be designed with protection against fire as a major consideration.

(5) Maximum security for vital recards can be achieved through the use of specialized heat-humidity resistant magnetic computer tape vaults or safes $[10],[11]$.

\section{Remarks}

(a) Minimize the storage of paper, wood, cleaning fluids, etc., in this area. Keep the area orderly, clean, and unencumbered so that the spread of a fire is slowed and fire fighting can be performed efficiently.

(a) A live cigarette ash which lands on a tape surface can cause instant tape damage and data losses.

(a) Tightly closed metal or plastic containers of fer short-term protection against flames and steam. Metal containers are preferable since they will not warp.

These considerations must include:

(a) The overall type of building and room construction; i.e., wood or metal frames, metal shelving and walls, etc.

(b) The combustibility of adjacent or surrounding areas due to their construction or their internal contents such as flammable chemicals, etc.

(c) The security measures which can be designed into the tape storage area; i.e., sprinkler systems, extinguisher systems, smoke detectors, and on-site guard personnel, etc.

(d) Fire department distance and accessibility.

(a) Typically, these vaults are designed to maintain their internal temperature-humidity ranges below $65.5^{\circ} \mathrm{C}\left(150^{\circ} \mathrm{F}\right)$ and $85 \% \mathrm{RH}$ for periods up to four hours, two hours, or one hour.

(b) The decision for the vault type is based on items $4($ a) to $4(d)$ above:

- For example, a four-hour vault should be used in a building with poor fire resistance, with many nearby combustible areas, few security devices, and a geographical location which is distant from the nearest firehouse, say in excess of ten minutes. 


\subsection{Fire Recommendations}

(6) Proper installation of fire and smoke detection/suppression systems can provide an important margin of safety for the personnel, the installation, the tape library, and archives [2], [23].

(7) Carbon dioxide $\left(\mathrm{CO}_{2}\right)$, Halon, and water can be used for direct fire fighting in a computer tape installation.

(8) Store duplicate copies of vital tapes in alternate locations.

(9) Properly wound tapes offer more resistance to fire and water damage.

\section{Remarks}

- A one-hour vault would be effective when placed in a fire resistant building with limited combustible materials or areas nearby, with a full security environment; i.e., sprinklers, smoke detectors, Halon systems, on-site guard personnel, and a fire department facility nearby and easy site accessibility.

(a) These systems can (and should) provide:

- Early detection capability through proper placement.

- Complete area coverage; this may include under floor and above ceiling coverage, as well as inside equipment, when feasible.

- Air conditioner fan shutdown or system switch to a smoke venting mode of operation.

- Strong, audible local signals, and remote off-site alarm signals if the installation is unat tended.

- Automatic computer shutdown capability.

- Activation of automatic fire extinguishing equipment.

(a) $\mathrm{CO}_{2}$ and Halon are clean and do not leave a residue. Tapes which have been made wet with sprinklers or hose water should be treated as outlined in the Water Recommendations section 1.8 .

(a) These duplicate or backup tapes should be produced and transferred of f-site as quickly as possible. Set up a firm schedule for this operation. (Also see sec. 1.2-(33).)

(a) A loosely or unevenly wound tape will allow more water to seep between windings and cause tape cupping and other distortions.

(b) Magnetic tape is a poor heat conductor; therefore, a properly wound reel with a very smooth pack has a better survival potential after being subjected to a heat environment than a loose pack. 


\subsection{Fire Recommendations}

(10) If recorded computer tapes have been subjected to fire and heat, try the following:

\section{Remarks}

Serious damage such as layer-to-layer adhesion and coating breakdown can occur if a combination of water and heat produces steam and high humidity in the tape pack. Temperature and humidities in excess of $65.5^{\circ} \mathrm{C}\left(150^{\circ} \mathrm{F}\right)$ and $85 \% \mathrm{RH}$ can cause media damage to occur. Note vault T-H

characteristics in section 1.7-(5).

(a) Separate out those reels of tape which appear to have sustained the least amount of physical fire or heat damage as soon as possible.

(b) Clean all fire debris, fly ash, and smoke residue from their canisters, wrap-arounds, and flange surfaces. Do this before opening the eanisters or wrap-arounds.

(c) Use the slowest speed transport available to perform at least two full wind-rewind passes. Instrumentation tape recorders which run at $0.762 \mathrm{~m} / \mathrm{s}$ (30 in/s) and which can accom modate $12.7 \mathrm{~mm}(0.5 \mathrm{in})$ computer tapes are a good choice. Inspect the tapes as they are being wound. If they are badly warped or display layer-to-layer adhesion which is damaging the coating, or are shedding significant amounts of coating debris, the chances for data recovery are poor. Clean the transport af ter each tape is run.

(d) Give the promising tapes two full cleaning passes on a tape cleaner/winder (pref erably constant tension type). Rewind the tapes onto clean or new reels and make new labels.

(e) Relax these rewound and cleaned tapes for 24 to 48 hours in the normal operating environment (see sec. 1.4).

(f) Perform a read (and recopying) pass (see sec. 1.2-(23)). If the tapes will not load onto the transport at this time, store them in a low humidity environment and retry at intervals.

(g) Repeat item (a) above for next least damaged group of tapes. 


\subsection{Fire Recommendations}

(11) When a reel of tape is subjected to high temperatures, it is advantageous to use reel hub materials which have the same thermal coefficient of expansion as the tape pack materials [18].

\section{Remarks}

(a) Aluminum is an example of such a hub material. Equal expansion or contraction reduces the increases or decreases in tape pack pressure with changes in temperature. 


\subsection{Water Recommendations}

(1) In the af termath of water inundation of the tape area, quickly separate the wet and dry tapes. Also separate the vital records from the less important ones [17].

(2) Check all wet tape labels and make sure that they are legible.

(3) Begin a general drying of the entire storage area, including shelves, floors, canisters, safes, and vaults.

(4) The tape drying process should begin immediately.

\section{Remarks}

(a) This will organize the salvage operation and permit a more rapid media recovery. If possible, move either the wet or dry tapes out of the storage area; shift whichever group consists of a smaller number of tapes. Move all tapes quickly out of standing water areas. Note: Beware of live electrical power sources during these activities?

(a) Replace or make existing labels legible.

(b) Do not paste new label over the wet original.

(c) If the library is under a Tape Managem ent Systems control, see section 4 .

(a) Standing water may continue to inundate the tapes particularly if the water has entered a closed tape safe or vault; dry these areas immediately.

(b) Quickly open, check, and drain any water which may have entered the tape canisters.

(c) Tape reel hubs are of ten capable of trapping and holding water; check for this; shake and rotate reel to empty this water.

(d) Use wet-dry vacuum cleaners to absorb all standing water wherever accessible.

(a) Do not replace wet tapes into their canisters; this allows the air drying process to begin.

(b) Hand dry all external wet surfaces which are accessible.

(c) Do not force dry the wet tape pack with a heated airflow; this can cause high internal humidity which can lead to binder damage and layer-to-layer adhesion.

(d) Gently, separate the reel flanges with spacers such as rubber grommets to allow airflow through the tape pack-flange interface. This will reduce the probability of tape-to-flange sticking damage when the tapes are first run. It will also permit additional water runof $f$ from the vertically standing tapes. 


\subsection{Water Recommendations}

(5) The most effective drying phase begins when the individual tapes are run reel-to-reel on a device such as a tape cleaner or winder (see sec. 3.2.1).

(6) Try data recovery methods even af ter a period of total water immersion. 10

\section{Remarks}

(e) If possible, maintain a forced, room temperature airflow through this tape-to-reel configuration.

(a) Never at tempt to run wet tape on a regular tape drive. The tape will not perform correctly in the vacuum columns and there is a strong possibility that the wet tape will adhere to the column walls or on to the capstan with ensuing tape tearing or other damage.

(b) Run over cleaning tissues only, not over the blades.

(a) The following process successfully reactivated recorded tapes which were totally submerged for 12 hours in unclean river water: 11

(1) After all accessible surfaces were hand dried as described in section 1.8-(4), the tapes were stored in the normally recommended temperature-humidity environm ent (see sec. 1.4) for 48 hours.

(2) The tapes were then run for six or seven passes on a tape dry cleaner unit over tissue cleaners only.

(3) When tapes were reasonably dried, then two cleaning passes were performed over tissues and cleaning blades.

(4) The tapes were then immediately read and recopied onto new reels.

NOTE: Binder degradation due to the water absorption was not yet apparent at this time, although the potential for this to occur eventually was considered to be a cause for concern. Therefore, recopying was considered to be important by the company. It was further noted that an ideal cleaning-drying unit for this river submersion (or any dirty water submersion) event would have been a tape cleaner which used a wet cleaning process. A number of tapes which had been submerged in salt water for a period of time were also successfully reactivated. The same recovery process was applied.

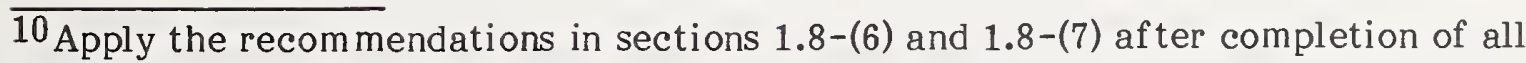
prior steps for all wet tape cases.

${ }^{11}$ Performed by General Kinetics, Inc. 


\subsection{Water Recommendations}

(7) The processes described in section 1.8-(6) are directly applicable to tapes which have been subjected to either clean or dirty water inundation.

(8) Avoid placing the tape racks, safes, and vaults in the lowest geographical locations. Locate the computer tape repository at a floor level which reduces the potential for flooding while allowing for effective fire fighting.

(9) Have plastic sheets available to throw over the tape racks, disk packs, and equipment in the case of a fire threat in which water will be applied by hoses or sprinkler systems.

\section{Remarks}

(b) In the case of short-time clean water inundation, it is probably sufficient to replace only the key tapes. Information relative to the long-term hygroscopic effect (see sec. 7.1) on the binders of previously fully submerged tapes is not presently available to the author.

(a) For clean water inundation, the two blade cleaning passes of item (6)(a)-(3) may be omitted.

(a) Areas such as basements have the greatest flood potential.

(b) Keep the lowest tape shelves at least one foot above the floor in low locations. This is advantageous for both cleanliness and protection from water damage.

(a) When checking the fire fighting equipment, make sure to check the location and condition of these plastic sheets. 


\subsection{Effects of Magnetic Fields on Recorded Magnetic Media}

An uncontrolled environment may consist of what a top administrator in one of the largest Federal tape user agencies described as "walking a couple of thousand (computer) tapes a day back and forth between two buildings." It may also consist of a passage of recorded computer tapes through modern airport terminals or environmentally uncontrolled warehouses, vehicles, and other storage and work areas. The potential for tape data erasure while in airports, has been of particular concern because of the magnetic fields in the anti-hijacking-metal detection devices, the airport radar signal fields, and the $\mathrm{x}$-ray energy in the surveillance equipment at the entry check points.

The information in sections $1.10,1.11$, and 1.12 has been derived from tests in which recorded computer magnetic tapes, cartridges, cassettes, and credit cards were subjected to various types of fields [13].

These tests showed that magnetic field energy was capable of causing subtle and serious data erasure on computer magnetic tapes, cartridges, cassettes, flexible disks, and credit cards with no observable, physical deterioration of the media itself. The erasing potential of magnetic fields is expected since all recording, erasing, and overwriting processes on the aforementioned computer magnetic media are performed by these fields.

If a magnetic field is of sufficient intensity to completely erose recorded data, it will do so almost instantaneously (i.e., in approximately $10^{-9} \mathrm{~s}$ ). Conversely, if a magnetic field is of insufficient magnitude to compietely erase the data, it will not do so no matter how long the recorded media is in the field.

To completely erase a recorded signal, it is necessary for the strength of the erasing field to be greater in value than the coercivity of the medium. The coercivity of magnetic media is that quality of the media, rated in amperes per meter (oersteds) which enables it to resist erasure of its recorded data. Typical coer civity values for present day computer magnetic tapes range from $22,000 \mathrm{~A} / \mathrm{m}(270$ Oe) to $30,000 \mathrm{~A} / \mathrm{m}(375 \mathrm{De})$. Coercivities on the order of $48,000 \mathrm{~A} / \mathrm{m}(600 \mathrm{Oe})$ are being produced in the new high energy tapes. For example, a magnetic field strength of $4,000 \mathrm{~A} / \mathrm{m}(50 \mathrm{Oe})$ was found to decrease the level of a recorded tape signal by approximately $2 \%$ below its original level (see fig. 2.). Computer tape data is typically not lost until its level has been decreased to $50 \%$ to $65 \%$ below its original signal level, dependent upon the system (i.e., NRZI or PE recording). Under some conditions, 246 bpmm (6250 bpi) (GCR recording) will not lose data even for greater signal level losses than the preceding systems. It is of interest to note that the earth's magnetic field strength is approximately $40 \mathrm{~A} / \mathrm{m}(0.5 \mathrm{Oe})$.

It was found that large, portable, and concealable permanent magnets were unable to erase any data from recorded computer magnetic tapes at a distance greater than $76 \mathrm{~mm}$ (3.0 in). However, a typical small of fice bar magnet caused a loss of signal level when it was placed into direct contact with the side of the tape reel flange. As expected, the loss was greatest on the edge tracks which are closest to the magnet.

Magnetic fields produced by alternating current flowing through the windings of a motor, generator, or transformer have the same tape erasure capability as those of 
equal strength produced by a permanent magnet. However, tests showed that so long as these units were properly encased in their typical magnetic shielding materials they produced no data losses on tapes placed in direct contact with the cases (see sec. 1.11, Question 6).

Figures 1. and 2. (reference [13]) show the erasing effects on the recorded signal level on a computer tape caused by both a large permanent magnet (see sec. 1.11, Question 7) and a low frequency $(60 \mathrm{~Hz})$ varying magnetic field. The abscissae in figure 1. is the distance along tape surface as measured from the BOT. Note that the erasure effect is greatest at the outermost tape layers which are in the closest proximity to the permanent magnet (i.e., distance d), and decreases with distance along the tape surface. Curve A has an $11.1 \mathrm{~mm}(0.44 \mathrm{in})$ tape-to-magnet spacing, d; curve $\mathrm{B}$ has $25.4 \mathrm{~mm}$ (1.0 in) tape-to-magnet spacing, d; and curve $\mathrm{C}$ has a 50.8 $\mathrm{mm}$ (2.0 in) tape-to-magnet spacing, $d$. The peak value of a varying magnetic field is the quantity which causes the observed data erasure effects. 


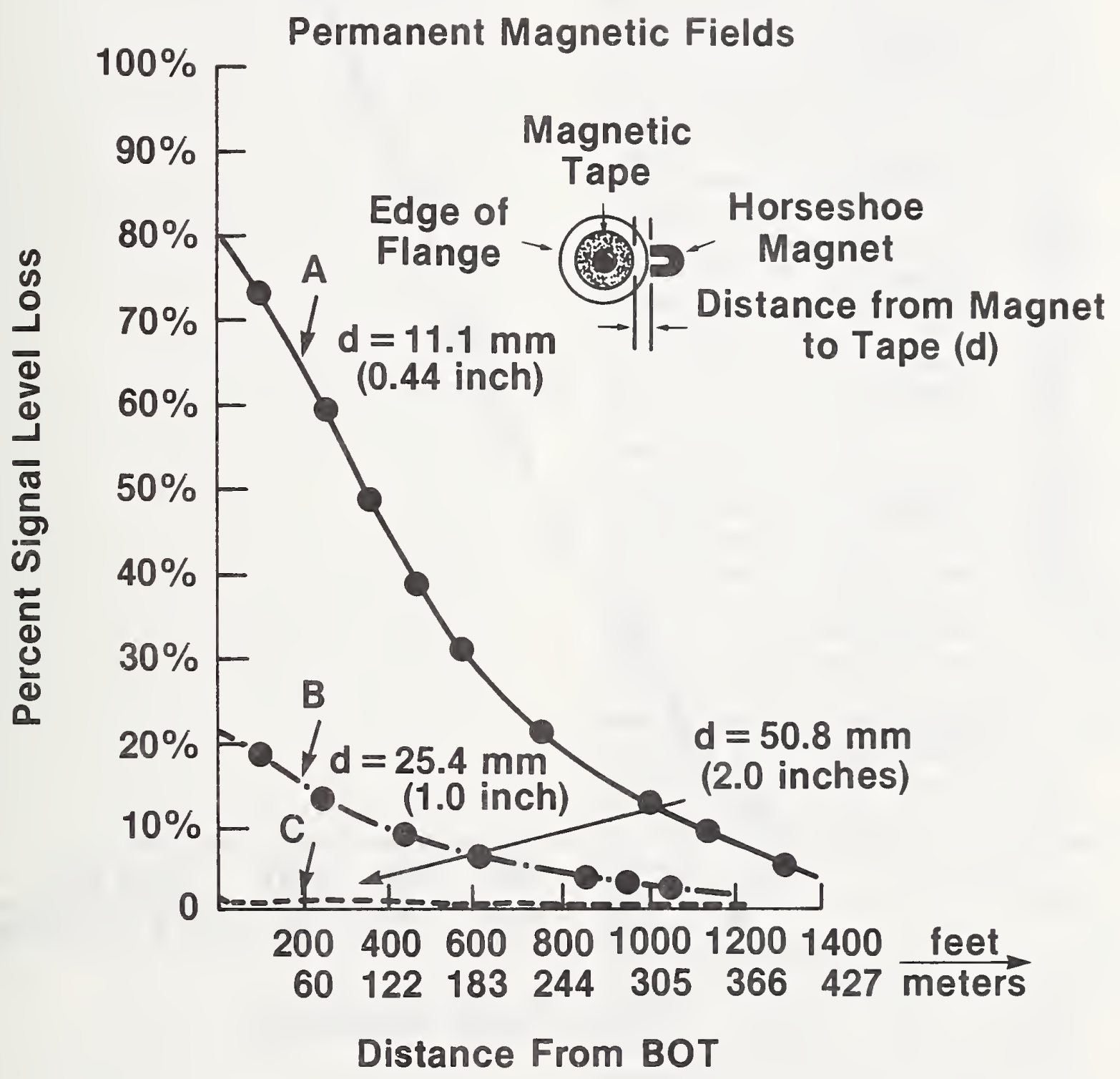

Fig. 1 


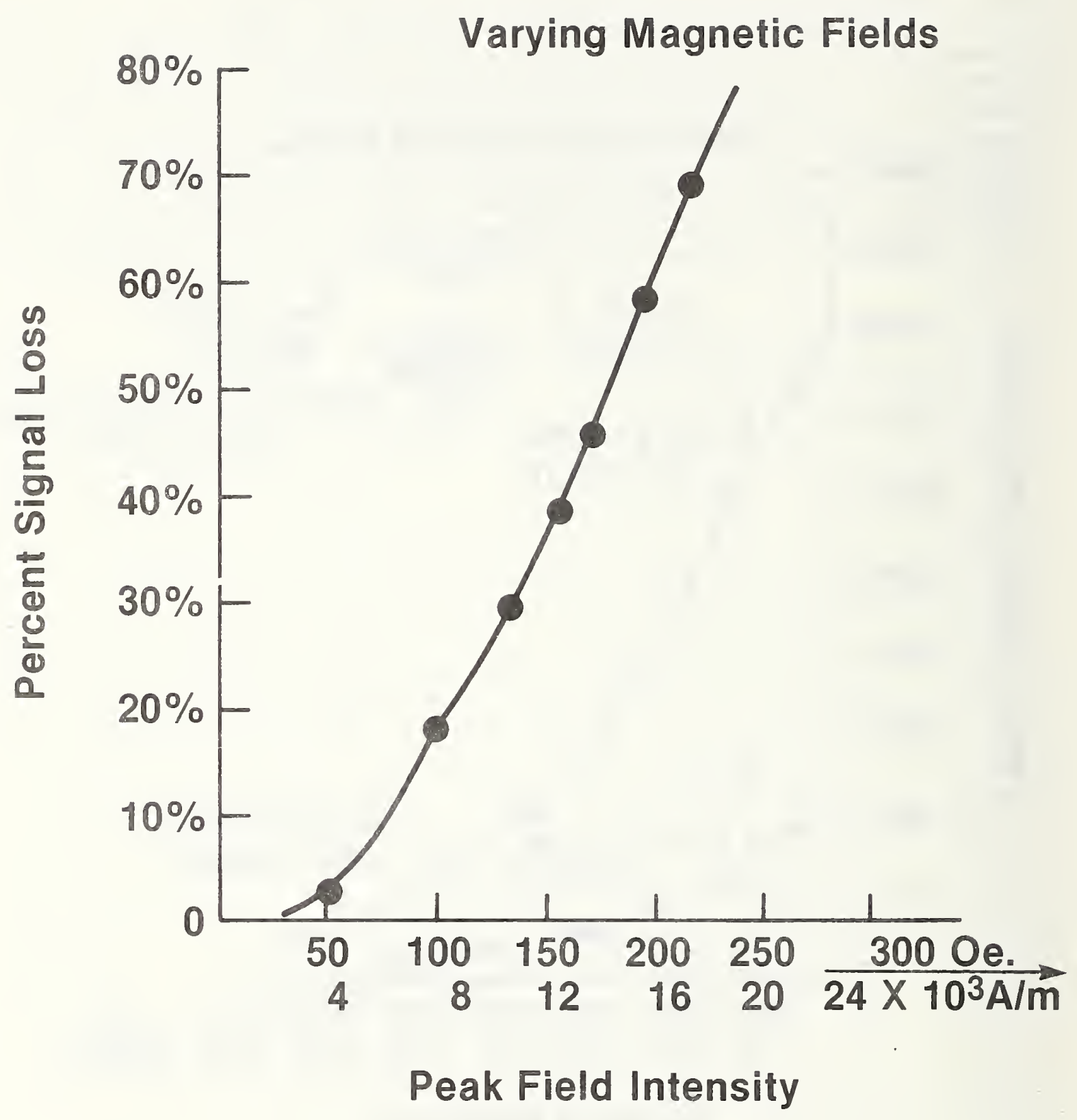

Fig. 2 


\subsection{Exposure to Fields and Devices Recommendations}

(1) Magnetic or electromagnetic fields: special precautions required.

(2) Airport metal detectors: no special precautions are required.

\section{Remarks}

(a) See sections 1.11 and 1.12 for a complete discussion.

(a) Many airport anti-hijacking metal detectors are of the "active" type which fill their walk-through space with their own internally generated varying magnetic fields. Tests were performed on a number of different kinds of walk-through detectors in two airports. Recorded computer tapes were moved through many different locations within the dectors and then tested for a loss of data. There was no observed instance of data loss. The highest detector field intensity encountered during the tests was in a unit which was rated at $398 \mathrm{~A} / \mathrm{m}(5 \mathrm{Oe})$.

(b) Most of the detectors which will be encountered in the future will probably generate weaker fields. A voluntary performance standard for walk-through detectors has been prepared by the Law Enforcement Standards Laboratory of the National Bureau of Standards. This standard recommends that the allowable generated magnetic field in walk-through metal detectors used for law enforcement should be held to a maximum value of approximately $95 \mathrm{~A} / \mathrm{m}$ (1.19 Oe), a level much too small to cause erasure of magnetic media. This trend towards reduced field strength units is highly desirable because of factors which must be considered for detectors that are used with people who may have medical problems.

(c) Handheld metal detectors are sometimes used as backup surveillance devices in airports. Exposure to these handheld detectors produced no effects on the recorded data even when they were placed in direct contact with the media housings.

(a) Recorded magnetic computer tapes are of ten transported through areas in which radar antenna are transmitting signals. 


\subsection{0}

Exposure to Fields and Devices Recommendations

(4)

X-rays: no special precautions are required.

(5) High voltages: no special precautions are required.

\section{Remarks}

Recorded magnetic tapes were irradiated by L-band, C-band, and X-band radar systems; the $\mathrm{L}$ and $\mathrm{C}$-bands radiated 200,000 watts of peak power and the $\mathrm{X}$-band radar radiated 500,000 wat ts of peak power. The tapes were placed for 10 seconds directly into the radar signal paths at distances of $30.5 \mathrm{~m}(100 \mathrm{ft})$, $15.2 \mathrm{~m}(50 \mathrm{ft})$, and $3 \mathrm{~m}(10 \mathrm{ft})$ from stationary, non-scanning antennas. There was no loss of recorded data produced at any of these distances by any of the radar units. It is not likely that these radars could cause signal erasure unless the media were at the point of maximum field strength, almost in contact with the antenna. The ineffectiveness of the radar electromagnetic field for erasing signals is due to the fact that the magnetic component of the electromagnetic field loses its strength very rapidly with distance from the antenna.

(a) Recorded magnetic media are of ten subjected to $x$-ray energy in airport surveillance systems. Tests were performed with various recorded magnetic media in the NBS Radiation Physics laboratory. The media were subjected to extremely high (lethal) $\mathrm{x}$-ray dosages. No data losses were incurred. Recorded media were also subjected to normal $\mathrm{x}$-ray inspection at two airports with no resultant loss of data.

(a) High voltages generated at the NBS High Voltage Laboratory were applied directly across recorded magnetic stripe cards. The laboratory voltage generator outputs were increased to more than 15,000 volts until air breakdown occurred between the electrodes. Arcs were struck directly onto the recorded magnetic stripes on the surfaces of the plastic cards. No signal or data losses resulted from these tests. This 


\subsection{Exposure to Fields and Devices Recommendations}

(6) Nuclear radiation (gamma rays):
no special precautions are
required.

(7) Automobile interiors: no special precautions are required.

(8) Television receivers: exercise some care although potential dangers to recorded data are slight.

\section{Remarks}

resistance to signal loss is valid so long as the high voltage fields do not produce excessive arcing which overheats or physically damages the media.

(a) A recorded digital magnetic tape cassette absorbed a gamma-ray dose of approximately $3.0 \times 10^{4}$ Gy $\left(3.0 \times 10^{6} \mathrm{rd}\right)$ in the NBS gamma-ray pool. There was no observed data loss on the media. It has been reported that data on recorded magnetic media was able to survive the effects of the electromagnetic energy component resulting even from nuclear detonation providing that the media materials are not damaged or otherwise altered by the radiated energy.

(a) Magnetic media carried within the passenger compartment of numerous automobiles experienced no data losses. Within the engine compartment, test cassettes experienced very little signal level decreases and no data losses even though they were put into contact with the ignition coil, generator, the starter motor, and the battery cable while starting the engine. Capacitive discharge electronic ignition systems produced no observable changes in the recorded signals.

(a) Television receivers are sources of magnetic fields, high voltages, and $\mathrm{x}$-ray energy. Tests were performed by placing recorded magnetic media both in the interior and exterior regions of different receivers. Media were placed in close proximity to the high voltage circuits which are also the sources of the x-rays. No signal level losses were observed, as anticipated by items (4) and (5) above. However, because of the many magnetic field producing components in television receivers and the many untested models, it is prudent not to use the cabinet tops as a storage space for recorded magnetic media. 
1.10 Exposure to Fields and Devices Recommendations

(9) Light and laser beams: no special precautions are required unless laser beam power is sufficient to burn (ablate) the media.

(10) Pressure: no special precautions are required other than avoiding the permanent physical distortion of the media.

(11) Static electricity: maintain a proper temperature and humidity and a clean environment (see secs. 1.3 and 1.4).
Remarks

(a) kecorded media were subjected to light sour ces ranging from inf rared through an intense ul traviolet light source with no resultant data loss. The radiation from a laser beam also has no effect on the stored data if spread over a large area on the surface of the medium. However, a sharply focused laser light beam can be made sufficiently intense to either heat or destroy the physical medium itself.

(a) Magnetically encoded plastic credit cards are of ten subjected to pressures in the pocket, wallet, or in cases. The effects of pressure were tested by placing $900 \mathrm{~kg}(2,000 \mathrm{lb})$ of weight on a number of individual credit cards. There was no loss of magnetically encoded data. Normal pressures have no effect on the recorded data on horizontally stacked flexible disks provided that physical damage does not occur.

(a) Excess static charges due to tape friction in a low humidity environment can cause:

- Tape dragging and skew.

- Tape flutter and signal variations.

- Increased tape and head wear.

- Recorded noise and data errors (caused by arcing); with no effect on the prerecorded magnetic information on the tape.

- Dust at traction.

(b) Static charge ef fects are greatly diminished (by the manuf acturers) by:

- Adding conductive material to the tape binder chemistry which reduces the resistance of the coating. This prevents a deleterious charge buildup. (Required coating resistivity values are greater than $5 \times 10^{5}$ ohms per square but less than $5 \times 10^{8}$ ohms per square).

- Reducing the friction in the tape path by using special materials which minimize tape contact; for example, beaded-glass surfaces in the vacuum columns.

- Grounding points in the tape path. 


\subsection{Questions and Answers Relative to the Effects of Magnetic Fields on Recorded Media}

The following series of questions were posed by the Office of Management and Budget relative to the data damaging potential of various commonly used magnets and low frequency (i.e., $60 \mathrm{~Hz}$ ) magnetic fields. The answers were published in [14] and are applicable to all recorded magnetic media including tapes, cassettes, cartridges, and disks.

Question 1.

Could someone by putting a couple of the little office bar magnets into a plastic computer tape canister, and then by putting the canister onto a tape rack, erase and destroy the data on a significant number of nearby reels over a period of months?

1. The length of time that the magnets are near to the recorded computer tapes or other magnetic media has no relationship to their effectiveness in obliterating the tape data. If the fields from the magnets are capable of erasing the tape, they will do so as soon as the tape is within their effective range. In a test, these little bar magnets whose field strengths averaged over approximately 44,000 to $52,000 \mathrm{~A} / \mathrm{m}$ (550 to $650 \mathrm{Oe}$ ) were contacted singly and in pairs directly against the flange of a recorded reel of computer tape. It was found that they were able to cause as much as a $35 \%$ decrease in the recorded signal amplitude on the track next to the edge track when used in pairs. Question 1 as posed, however, indicates that these magnets are to be placed into an adjoining tape canister. This reduces their erasing effects by moving the tape into a much weaker part of their fields. It was found that if the tapes as well as the magnets are both enclosed in individual plastic cases then the erasing effect is undet ectable for these bar magnets.

\section{Question 2.}

Could someone put a $51 \mathrm{~mm} \times 102 \mathrm{~mm}$ ( 2 in $\times 4$ in) magnetic of fice picture hook in his pocket, walk by a tape rack, and destroy the data on a significant number of tapes in the rack?

1. The maximum field strengths close to the surface of each of a number of magnetic picture hooks was measured as 35,800 to $40,000 \mathrm{~A} / \mathrm{m}$ (450 to $500 \mathrm{Oe})$. They had no measurable effect on the signal when carried in a pocket in the vicinity of a recorded reel of tape. One of these magnets was firmly pressed directly against the exposed flange of a reel of tape. The track next to the edge track experienced an $8 \%$ decrease in signal amplitude. The magnetic picture hooks are less effective than the bar magnets in Question 1, because their internal array of flat magnets do not tend to produce fields that are as strong at a distance. The test was repeated with the tape enclosed in its plastic canister and with the magnetic picture hook strapped to the canister surface for two days. No observable signal loss occurred at this distance. 
Question 3.

Could someone attach the aforementioned picture hooks magnetically to the tape rack in an obscure place and destroy the data on a number of tapes over a period of a month?

1. This question has been answered by the previous comments. Time is no element in the destructive process; only the maximum magnetic field strength and proximity to the tape are important. The picture hook out of direct contact with the flanges on the reels of tape is ineffective as a signal erasing device. As a matter of fact, when a permanent magnet is fastened to a metal rack surface by its own magnetic attraction, its fields tend to be diverted or shielded by the metal rack and are even less effective than before at any distance from the magnet (see sec. 1.12-(2)).

\section{Question 4.}

Given a one-story building housing a tape storage area, could someone place a permanent magnet weighing, say $18 \mathrm{~kg}(40 \mathrm{lb})$ on the roof and have any effect on the data on the tapes?

1. The far field intensity of a magnet will decrease approximately by the inverse cube of the distance from its poles. At the present time, there are no permanent magnets available that are known to the author that can erase the information from a magnetic tape that is placed several feet away from the magnet.

\section{Question 5.}

Given a first floor installation separated from the street by a $6.1 \mathrm{~m}(20 \mathrm{ft})$ sidewalk, with the tape racks $30.5 \mathrm{~m}(100 \mathrm{ft})$ from the curb, could someone place an electromagnet (of the type used in junk yards to load scrap) into a van, connect the magnet to a series of automobile storage batteries in the van, drive up to the curb outside the computer room and, in say a minute, wipe out the entire installation's files and programs?

1. This experiment was actually simulated at a local scrap metal (junk) yard. Four recorded tapes were placed into the field of a scrap lif ting magnet at various distances: at $0.41 \mathrm{~m}(1.3 \mathrm{ft})$, at $1.52 \mathrm{~m}(5 \mathrm{ft})$, and at $3.05 \mathrm{~m}(10 \mathrm{ft})$. At $0.41 \mathrm{~m}(1.3 \mathrm{ft})$, a uniform signal level reduction of approximately 5 percent was observed. At $1.52 \mathrm{~m}(5 \mathrm{ft})$ and above, no observable reduction occurred. According to the crane operator, the magnet had a lifting capacity of approximately $363 \mathrm{~kg}(800 \mathrm{lb})$ of scrap metal. However, the gap structure of a lifting magnet is such that there is little distant magnetic field strength projecting outward from the magnet pole faces. This type of a lifting magnet is effective primarily upon contact with the material and cannot perform at a distance as suggested.

2. Now consider a non-lifting type of electromagnet: a magnetic field forms around any wire through which electric current flows. This field can be made very intense by shaping the wire into the form of a coil or "solenoid" and increasing the current flow. However, increased current flow requires 
more power from the electrical supply as well as special cooling systems for the coils. Coils that are specifically designed for producing very strong magnetic fields that are in the range from approximately 8 to 40 million $\mathrm{A} / \mathrm{m}(100,000$ to $500,000 \mathrm{Oe})$ are not designed to produce fields at long distances. They are constructed to produce concentrated magnetic fields in the center region of the coils and are used to perform basic scientific experiments on materials that are placed into the most powerful regions of the coil field. For example, one known12 high intensity water-cooled magnet coil which has approximately a 12 million $\mathrm{A} / \mathrm{m}(150,000$ Oe) field near its center drops to less than approximately $80,000 \mathrm{~A} / \mathrm{m}(1,000 \mathrm{Oe})$ within $0.305 \mathrm{~m}(1 \mathrm{ft})$ of the coil center. This magnet requires a power supply that is capable of supplying at least 3 million watts of electrical power to the coil. A magnet system such as this is impossible to transport in any ordinary vehicle.

\section{Question 6.}

Is there any way to shield the information recorded on a magnetic tape from an outside magnetic field produced by a magnet or a motor?

1. It has been noted (see sec. 1.9) that recorded information on a magnetic tape can be shielded from the effects of a magnetic field by keeping the tapes at a relatively small distance, i.e., $76 \mathrm{~mm}$ ( $3.0 \mathrm{in}$ ), from a source such as a permanent magnet or motor. It is also possible to shield the tape more effectively by placing it either into an individual container or into a vault whose surfaces are made of sof $t$ ferromagnetic shielding materials (i.e., materials which do not become permanently self-magnetized). These materials are conductors of both electricity and magnetic flux and will divert the magnetic field. That is, most of the magnetic field energy is absorbed in the shielding material and very little passes through it into the air on the opposite side. The shield is usually fabricated from some ferrous or ferrite material in a number of different physical forms such as sheets, rolls, and coatings of various thicknesses. Examples of effective shielding materials for magnetic fields are given in reference [24]. A multi-layer shielding structure can be devised by using the proper combinations of materials and air space. For example, there are shielding materials which are designed for reducing the strength of very powerful magnetic fields while others are designed to absorb weaker magnetic fields. Therefore, a layer of one material can be placed close to the outside of a tape enclosure in order to absorb most of the impinging magnetic field. This can be followed first by an air space, and then by a layer of the other material close to the interior wall of the enclosure which will absorb the remaining field.

There are several organizations whose primary function is the development, design, and production of magnetic shielding environments. It is a worthwhile idea to consider using their information services $[10],[11]$.

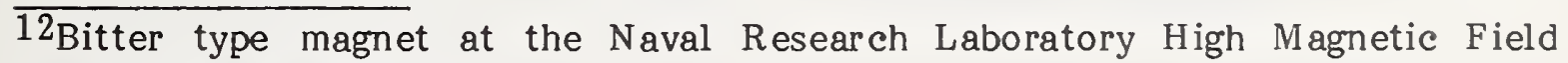
facility, Washington, D.C. 
A potential source of unwanted magnetic fields such as a large motor, generator, or transformer that is near the tape installation should also be enclosed in shielding materials. The shield layer or layers should be at least $6.4 \mathrm{~mm}(0.25 \mathrm{in}$ ) or more away from the internal device and should surround it completely and be sealed properly. Whenever possible, the initial design and placement of a tape repository should consider and avoid sour ces of un wanted magnetic fields.

Question 7.

Are there magnets available that are much more powerful than the little office bar or picture hook magnets but that are still easy to conceal and to carry about?

1. Yes, there are. Tests were performed with a concealable magnet which weighed approximately $0.80 \mathrm{~kg}(1.75 \mathrm{lb})$ and had a maximum center field strength value of approximately $56,000 \mathrm{~A} / \mathrm{m}(700 \mathrm{Oe})$. The test results showed that this magnet was extremely effective when it contacted the outer flange edges of a recorded computer tape reel which was stored in a normal vertical position; i.e., the signal on the first $210 \mathrm{~m}(700 \mathrm{ft})$ of the tape was badly damaged. The intrusion of these magnets into a tape storage area must be avoided at all costs! 


\subsection{Magnetic Field Recommendations}

(1) For protection against data erasure, do not allow any permanent magnets to come closer than $76 \mathrm{~mm}(3.0 \mathrm{in})$ to a recorded magnetic media surface. Maintain strict magnet control in all areas in which recorded magnetic media are found. This includes in the office and in tape operating or storage areas!
(2) Vaults, canisters, carrying containers and cabinets made of soft magnetic shielding materials offer effective protection against data erasure by external magnetic fields at close distances.

\section{Remarks}

(a) Stray magnetic fields which are of sufficient intensity to obliterate data on computer magnetic tapes at a distance greater than $76 \mathrm{~mm}$ (3.0 in) from the source are rarely (if ever) encountered in normal environments.

(b) All protective measures decribed in this section are equally effective against the low frequency varying fields produced by motors, transformers, generators, etc. The type of field is not as significant in its data erasure potential as the peak value to which the varying field subjects the recorded media.

(c) Recorded magnetic media of all types can be placed as close to each other as desired with no effects on their recorded data.

(a) The shielding materials should have high permeabilities and be magnetically soft. That is, when these materials divert the external static and low frequency varying magnetic fields in order to protect the stored recorded media, they must not become magnetized, i.e., retain their own residual magnetism. Examples of good "sof t" shielding materials are various alloys including those with the proper proportion of nickel-iron, nickel-iron copper, and certain grades of magnetically sof $t$ steel [24].

(b) If the container size is a factor, the surfaces of vital tapes can be spaced to within $25.4 \mathrm{~mm}$ (1.0 in) from the inside walls of the container provided that these walls are covered with high permeability, sof $t$ magnetic shielding materials; there are specialized small carrying containers for this application.

(c) In addition to the carrying cases in (b), there are large ferromagnetically shielded storage cabinets available. These are effective for safe, long-term storage of vital tapes in the repository environment [10], [11]. 
(d) Non-shielded tape shipping containers should maintain a $76 \mathrm{~mm}$ (3.0 in) spacing between the media and the exterior walls.

(e) Recorded computer magnetic tapes are of ten subjected to very high frequency electromagnetic fields such as those produced by radar systems. Although these fields can be shielded against through the use of specialized screening techniques, such measures are not necessary (see sec. 1.10-(3)).

(3) Whenever possible, design and locate tape repositories away from components which produce magnetic fields.

(a) These components include large motors, generators, transformers, and electrical power lines carrying heavy current loads.

(b) Make sure that these components are magnetically shielded if they are in the repository area (see sec. 1.11 , Question 6 and sec. 1.12-(2)).

(c) It is claimed by some that the tapes should not be stored close to structural steel beams which can be magnetized by lightning strokes or other electrical currents.

(4) Initiate total security measures for access to media repositories particularly if vital magnetic tape records are stored in these areas.

(a) Permit only fully identified and authorized personnel into the tape repository area.

(b) If vital records are stored in the repository area, set up magnetometer checks in order to detect concealed magnets. For example, hand-held or walk-through magnetometers (with alarms) can be employed. Note that an incredible amount of intentional damage to stored data can be quickly accomplished with a powerful but concealable, hand-held permanent magnet (see sec. 1.11, Question 7). An additional danger stems from the fact that the data destruction may not be detected for a long period of time after the event; therefore, the sabotage may be an ongoing process.

(c) Advise all personnel never to carry any type of magnet into a magnetic tape repository; initiate severe penalties. 


\subsection{Magnetic Field Recommendations}

(5) Test the tape path's metal components for residual magnetism. These parts include read/write heads, guides, capstans, and rollers. Degauss (demagnetize) these parts as required. Alternately, degauss the components on a scheduled basis without testing.

\section{Remarks}

(a) Residual magnetization can cause some signal level reduction on recorded computer tapes as they pass over these magnetized components.

(b) There are hand-held magnetometers available for measuring the residual magnetism.

(c) There are hand-held degaussers available for demagnetizing the parts $[10],[11]$.

(d) Turn of the power to the tape drive and demount all tapes before measuring and degaussing the tape path components.

(e) The guides, rollers, and capstans will be more difficult to demagnetize than the $\mathrm{read} / \mathrm{write}$ heads since they are "harder" magnetically.

(f) In order to prevent head magnetization, avoid any operation which forces direct current through the head windings without an associated varying signal current. Also, do not touch the head or guides with a permanent magnet. 


\subsection{Scheduled Computer Tape Maintenance}

This is a difficult area in which to establish exact scheduling criteria since (a) the recommendations for tape cleaning, tape evaluation (or certification), and tape rehabilitation (see sec. 3.) are derived from diverse types of operations and sources and (b) there can be significant differences in the characteristics of tapes emanating from different and sometimes the same sources. At the present time, there are no accepted standards or agreements relative to the choice of scheduling criteria; the literature is replete with variations. When the source is a manufacturer of tape maintenance equipment or a media rehabilitation contractor, the recommended scheduled operations tend to be conservative and incorporate frequent tape care. When the sources are the users of the media, the recommendations are more heavily weighted by the economic considerations for contracting out or setting up and operating an in-house scheduled tape maintenance program. For example, one major tape user performs no tape maintenance unless trouble is encountered when attempting to read the tapes after removal from storage. Another user suggests that tapes should not be cleaned-instead it suggests that the beginning tape footage be excised when tape errors develop. Generally, small, high use libraries require more frequent care for each tape, while large tape libraries, in which individual tapes are usually not mounted as often, tend to require a lower maintenance frequency for each tape. However, the large tape user organization with a rehabilitation facility will usually institute criteria which tend to produce a steady flow of tapes into the rehabilitation process (see sec. 3.3). For example, one large Government tape user organization maintains a steady flow of 5,000 reels per day into their contractor's rehabilitation facility.

Section 1.14 presents a number of diverse scheduled tape maintenance options and recommendations. Individual organizations should institute those recommendations and options which are found to be most effective for their operations. For example, the previously mentioned large Government tape user found after a period of application, that the decision to rehabilitate tapes after they have been mounted a specified number of times was not an effective approach for their organization. Instead, their primary choice for rehabilitation candidates is now based on the tape data expiration dates, while their secondary choices are based on tapes which are troublesome; i.e., display data losses or operating difficulties. 
1.14 Scheduled Computer Tape Maintenance Recommendations

(1) Establish a schedule for cleaning magnetic computer tapes (see sec. 3.2.1.)
Remarks

Tape cleaning 13 recommendations from various sour ces: 14

(a) Problem tapes (marginal operation): 15 clean as soon as problems are observed. Recopy if necessary.

(b) New tapes: clean upon receipt or before first use.

(c) Tapes scheduled for long-term archival storage: clean before storage. Proper winding and tensioning is critical (see secs. 7.3 and 7.4).

(d) Large library: clean each tape af ter 5 to 15 mountings.

(e) Small library: clean each tape after every 1 to 3 mountings.

(f) Clean tapes when they develop a specified number of additional errors (say, 5 errors).

(g) Clean each stored or active tape approximately every 6 to 12 months.

(h) Clean tape only if data errors are encountered on the first read pass after storage; otherwise do nothing to the tape.

(i) Don't clean tapes which are being used only over a short length (starting at the BOT): cut off the used section of tape when errors develop.

(j) When beginning a scheduled cleaning program, clean each tape once for each year of prior usage.

(k) Do the cleaning and winding in the same $\mathrm{T}-\mathrm{H}$ environment as that in which the tapes will be stored. Acclimatize the tapes for 24 hours before cleaning if they have been transported into the cleaning area from a different $\mathrm{T}-\mathrm{H}$ environment (alternately apply sec. $1.5-(4))$.

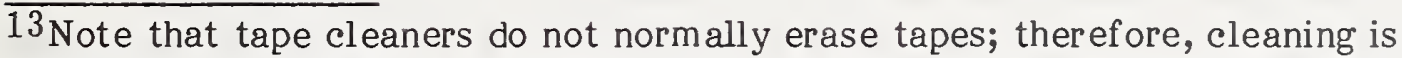
applicable to recorded or unrecorded tapes. However, make certain that the cleaner does not have an erase mode in an "ON" state.

${ }^{14}$ Note that there are no implied priorities for these recommendations; several may be instituted concurrently, if suitable.

15 Make certain that marginal operation does not stem from a system malfunction such as tape drive misalignment. 
1.14 Scheduled Computer Tape Maintenance Recommendations

(2) Establish a schedule for evaluating/certifying computer magnetic tapes (see sec. $3.2 .2-3.2 .3)$.
Remarks

Tape evaluation/ cer tification 16

recommendations (from various sources): 17

(a) Evaluate marginal tapes as soon as problems are observed. 18

(b) Evaluate active tapes after 3 to 6 cleanings.

(c) Evaluate each active tape after approximately 30 mountings.

(d) Evaluate (or certify) tapes within six months prior to use in long-term storage. Thereaf ter, evaluate these tapes once per year; non-destructive testing can be performed (see sec. 4.4).

(e) Evaluate new library tapes upon receipt (optional); alternate suggestion--clean only upon receipt (see sec. 1.14-(1)).

(f) Evaluate a tape after it has been relegated to scratch status or its data has been copied onto another reel, or when the data has expired and recopying is unnecessary.

(g) Evaluate a tape after it has developed a specified number of write skip errors.

(h) Evaluate the tapes in the same $\mathrm{T}-\mathrm{H}$ environment as that in which the tapes will be operated or stored. Acclimatize the tapes for 24 hours before evaluation, if they have been transported into the evaluator area from a different $\mathrm{T}-\mathrm{H}$ environment (alternately apply sec. $1.5-(4))$.

(a) Rehabilitation may be performed in-house or by a contracting organization.

(b) Contractors are a good source of information relative to the costs, and the pros and cons for setting up a rehabilitation program.

\footnotetext{
16 Evaluators (and certifiers) will usually erase all of the pre-recorded tape data; therefore, if necessary, recopy the data before evaluating. Note that there are error-logging software systems (for example, see sec. 4.4) that can evaluate data files by reading the data non-destructively and examining the records and fields for bad spots. It is advantageous to clean the tapes before evaluation.

17 Note that there are no implied priorities for these recommendations; several may be instituted concur rently, if suitable.

18 Make certain that the marginal operation does not stem from a system malfunction such as tape drive misalignment.
} 
1.14 Scheduled Computer Tape Maintenance Recommendations
Remarks

(c) Tape rehabilitation recommendations

(from various sources): 19

Rehabilitate tapes:

- After they have caused any operational problems. 20

- After they have been in storage for a specific length of time (this thins out the vault population).

- After the data have reached their expiration date.

- After they have become scratch library tapes.

- After the data have been copied onto other reels.

- After they have developed more than a specified number of write skip errors.

- After they have been mounted more than a specified number of times over a given period of time; e.g., more than 4 mountings within 60 days.

- After they have been mounted a specified number of times; for example, after 30 mountings.

(d) Typical rehabilitation activities (see sec. 3.3 for complete details):

- Tape cleaning only or cleaning and evaluation.

- Reel and label condition checks plus replacements if necessary.

- Tape condition checks including signal tests at specific densities, and tape error tests at specific densities.

- Tapes are rewound at tensions which are compatible with the normal operating tape devices.

- Quality assurance testing by contractor in order to minimize rejected tape lots.

$\overline{19}$ Note that there are no implied priorities for these recommendations; several may be instituted concurrently, if suitable.

20 Make certain that the marginal operation does not stem from a system malfunction such as tape drive misalignment. 


\subsection{Scheduled Computer Tape} Maintenance Recommendations

(4) Establish application categories for tapes which have been evaluated or rehabilitated.

\section{Remarks}

(a) Optional categories:

- Tape has less than 10 write skip or dropout errors: unlimited use other than long-term archives. Use new state-of-the-art tapes for archival purposes (see sec. 2.1.1-(1)).

- Tape has 10-20 dropout errors: use for daily applications.

- Tape has excessive dropout errors and apparently defective edge tracks: discard. Excessive errors may be defined by the economic breakpoint at which it is cost effective to replace the tape with a new tape (see sec. 3.1.1). 


\subsection{Flexible Disk Cartridges}

Flexible magnetic disk cartridges ("floppy" disks) employ mylar substrates and $\gamma-\mathrm{Fe}_{2}{ }^{0} 3$ particles or cobalt modified $\gamma-\mathrm{Fe}_{2}{ }^{0} 3$ particles in their magnetic surface coatings and are designed with similar coercivity and magnetization parameters as both regular and high energy computer magnetic tapes. The magnetic media are made slightly thicker than computer tapes; i.e., $0.075 \mathrm{~mm}(0.003 \mathrm{in})$ since they must rotate in a plane and maintain their surface rigidity. Unlike the rigid disks in which the read/write heads are designed to fly aerodynamically above the magnetic disk surface, the flexible disk read/write head operates in direct contact with the disk surface, with opposing pressure offered by a pad or a similar head from the opposite surface. Hence, head and media wear and debris problems occur due to the frictional effects. These wear problems are reduced through the use of lubricants either directly integrated into the coating chemistry, or by lubricants which are applied externally to the disk surface.

The most popular diameters for flexible disks at the present time are $203 \mathrm{~mm}$ ( 8 in) and $133 \mathrm{~mm}$ (5.25 in). New, small "micro-disks" with $76 \mathrm{~m}$ (3.0 in) to $102 \mathrm{~mm}$ (4.0 in) diameters are also becoming available. Special features are being designed into these micro-disks that include: automatic shutters which prevent contamination of the exposed media surface, rigid protective shells which do not flex or bend, and rigid center hub disks which provide for proper disk placement and head tracking.

The effects of various external fields (see secs. 1.9 to 1.12) and T-H environments on flexible disks are analogous to those on the computer tapes; however, the disks develop none of the tape pack winding pressure and tension conditions which cause many of the serious computer tape problems (see secs. 7.3 and 7.4).

Many of the care and handling recommendations for magnetic computer tapes in section 1. are also applicable to the flexible disk media and should be observed. 
1.16 Flexible Disk Care and Handling Recommendations

(1) Media cleanliness is important. Maintain a clean working environment.

(2) Never make finger contact with the exposed media surface through the elongated, oval slot in the envelope where the head-to-disk access (contact) is made. Also avoid touching the exposed media surface area near the hub.

(3) Clean the flexible disk drive regularly particularly at its insertion slot position.

(4) Clean the read/write heads on a regular basis.

\section{Remarks}

(a) Contamination of the flexible disk surface is a serious cause of data losses. Finger oil, smoke, food and drinks, abrasive materials (such as dust or filings), pencil eraser debris, etc., are typical contaminants. Read/write heads last longer when used with clean media.

(a) These slotted regions of fer access to the media surfaces by contaminants particularly fingerprint oils and dirt.

(b) Some of the new state-of-the-art micro-sized flexible disks which have $76 \mathrm{~mm}$ (3.0 in) to $102 \mathrm{~mm}$ (4.0 in) diameters have automatic shut ter mechanisms which have been designed to prevent this finger contact problem.

(a) Dirt and dust can be picked up by the disk during insertion.

(a) Dilute isopropyl alcohol on a lint-free swab will clean contaminants from the head. Exert minimum force on the delicate tension springs.

(b) Cleaning kits are available which employ flexible disks made of a special material as the rotating media rather than the magnetic media of the regular disks. The cleaning material is either run dry or is wetted with a cleaning fluid; the head is then lowered onto the surface and cleaned under rotation. 21

(c) One kit manufacturer suggests two cleanings per week, another suggests a cleaning af ter each 40 operational hours, while others recommend a daily cleaning. Others suggest cleaning the flexible disk as soon as data errors appear.

\footnotetext{
21 Note: Some flexible disk head and drive manufacturers will invalidate their warrantees if some types of head cleaners are used. Contact individual manufacturers for head cleaning information.
} 


\subsection{Flexible Disk Care and Handling Recommendations}

(5) Always return the flexible disk into its protective envelope after it is used.

(6) Don't flex or fold the flexible disks. Avoid bending the disk when loading into or extracting it from the drive.

(7) Don't put a rubber band or a paper clip onto the flexible disk envelope.

(8) Always write on the label before it is applied to the disk. However, if the original label is used, do not write on it with a ball point pen; use a soft, fiber tipped pen.

(9) Never erase the label on a flexible disk cartridge. Cross out previous information; remove and replace label when full.

(10) General environmental requirements:
(a) Operation and storage:
Temperature: $10^{\circ} \mathrm{C}$ to $51.6^{\circ} \mathrm{C}$ $\left(50^{\circ} \mathrm{F}\right.$ to $\left.125^{\circ} \mathrm{F}\right)$
Relative humidity: $8 \%$ to $80 \%$
(b) Test environment:
Temperature: $20^{\circ} \mathrm{C}$ to $26^{\circ} \mathrm{C}$ $\left(68^{\circ} \mathrm{F}\right.$ to $\left.78^{\circ} \mathrm{F}\right)$
Relative humidity: $40 \%$ to $60 \%$
(c) Transportation:
Temperature: $-40^{\circ} \mathrm{C}$ to $51.6^{\circ} \mathrm{C}$ $\left(-40^{\circ} \mathrm{F}\right.$ to $\left.125^{\circ} \mathrm{F}\right)$

\section{Remarks}

(a) This prevents contamination and physical damage to the media. Don't lose the envelope. Don't scatter the disks around on table tops and desks in a random fashion.

(a) Although the envelope may recover, the internal media may be damaged permanently; a warped disk does not rotate properly.

(b) There are new micro-sized flexible disks which are enclosed in rigid plastic envelopes which do not bend or flex.

(a) The rubber band may cause the disk to remain bent permanently after long-term storage. The paper clip may emboss the media and cause permanent data losses due to head-to-media separation.

(a) A ball point pen can emboss the surface of the disk and cause permanent surface defects and data losses.

(a) Erasure can be a source of contamination. In fact, don't write on the label with a lead pencil. Don't paste a new label over the old label and do not cover any flexible disk holes with the new label.

The following are A merican National Standards Institute (ANSI X3.73-1980) recommendations:

(a) Disks should be conditioned for at least one hour in the operating environment before use.

(b) Disks should be acclimatized in the test environment for 24 hours before testing.

(c) In the cases of items (10)(a), (b), or (c), no moisture should be permitted to condense in or on the cartridge. 
1.16 Flexible Disk Care and Handling Recommendations

(11) Failure to observe the $\mathrm{T}-\mathrm{H}$ guidelines in item (10) can result in media damage. Be careful about the the storage location.
(12) Discontinue the use and acquisition of flexible disks which appear to shed excess debris and cause rapid head wear.
(13) Never place a magnet of any kind onto the envelope surface of a flexible disk cartridge! See sections 1.11 and 1.12 for the effects of magnetic fields on data recorded on flexible disks.

(14) For long-term storage, the flexible disk cartridges should be stored in a vertical position in a ten-disk storage container.

\section{Remarks}

(a) Acceptable temperatures and humidities are normally maintained in an operating area such as an office. However, the temperatures in a closed, hot, stationary, automobile in the sunlight may exceed the upper limits. Excessively cold temperatures may make these disks rigid. Acclimatize these affected disks in a normal $\mathrm{T}-\mathrm{H}$ environment (see sec. 1.16-(10)) for several hours before using.

(b) Don't use a flexible disk as a place mat.

(a) They may have abrasive surfaces which create considerable friction and heat and usually have short operating lives. The wear rate for a flexible disk compared to a computer tape is accelerated by the fact that the disk heads will pass over and make contact with the same point on the disk surface typically five to six times per second. A rapid decrease in the disk signal level may indicate a rapidly wearing disk surface. Built-in disk coating lubricants reduce this wear effect.

(a) These include typical bar magnets found in offices, coat and picture hangar magnets, flashlight magnets, magnetized screwdrivers, etc.! Keep magnets at least $76 \mathrm{~mm}$ (3.0 in) away from recorded disks. This applies to both rigid and flexible disks.

(b) Exercise complete magnet control in the of fice and the data processing work place.

(a) The vertically stored disks should be supported, by the container so that they cannot lean or sag. Make certain that no pressure is exerted against the disk envelopes.

(b) For safest storage, do not use a cardboard container; use a more rigid type, such as a hard plastic unit. 
1.16 Flexible Disk Care and Handling Recommendations

(15) For short-term storage, the flexible disk cartridges may be placed either vertically or horizontally in stacks of ten or less.

(16) Reinfor cing flexible disk hub rings: should they be installed by the user?
(17) Miaintain a schedule for checking, cleaning, or replacing associated air filters.

(18) Use the proper type of tab to cover the write protect notch on the disk envelop.

(19) Make copies of vital or master flexible disks and store in alternate locations.

(20) Electrical discharges produced by static electricity cannot damage or alter the data which has been correctly recorded and stored on the flexible disks.

(21) Inspect and replace worn head pads if necessary.
Remarks

(a) Do not put a weight or other pressure on these horizontal disks. There are many specialized rigid containers available for short and long-term storage and shipping of magnetic disks [12].

(a) Special rings have been developed for the purpose of reducing both the slippage of the flexible disk and inner media hole damage under rotational conditions [12]. At present, the question of the efficacy of these user installed rings and their possible deleterious effects is unresolved for the larger diameter $203 \mathrm{~mm}$ ( $8.0 \mathrm{in}$ ) and $133 \mathrm{~mm}$ (5.25 in) flexible disks.

(b) Micro-disks with $76 \mathrm{~mm}$ (3.0 in) to $102 \mathrm{~mm}$ (4.0 in) diameters, which have recently been introduced into the market, are manufactured with a rigid hub material adhesively bound to the mylar surface. These appear to eliminate the need for these user installed hub rings.

(a) See recommendations for clean tape operations in section 1.3. Dirt causes data losses and media damage.

(a) Don't use any tab material which leaves a sticky residue; use the tabs which are supplied with the disks.

(a) Do this as soon as possible before problems arise.

(a) However, electrical noise produced by arcing due to static electricity, may induce unwanted pulses into the sensitive electronic system.

(b) See sections 1.10-(5) and 1.10-(11).

(a) A worn pad may cause rapid debris formation, and a change in signal level. 


\subsection{Computer Tape Transport Care}

(1) A computer tape transport can cause immediate or delayed tape and data damage. Preventative and immediate maintenance is mandatory. 22

\section{Remarks}

Check the following:

(a) Read/write heads:

- Clean? See table 1. and section 1.3-(12).

- Burrs, scratches, etc.? Replace head.

- Gap smeared? (i.e., gap material from one side of the head gap shorts across to other side of the gap); replace head.

- Aligned? Use master skew tape.

- Magnetized? See section 1.12-(5).

- Brown-stain? See section 7.2(d).

(b) Tape path:

- Worn or dam aged guides or capstans? Replace.

- Aligned? (Misaligned guides or capstans can cause media damage.)

- Clean? See table 1. and section 1.3-(12).

Clean entire path including vacuum threading channel columns. Do not touch capstans with fingers. Remove bits of debris and tape from the bottom of the vacuum columns.

- Lubricated as recommended?

- Magnetized components? See section $1.12-(5)$.

(c) Tape dynamics:

- Tapes breaking, stretching, or cinching?

- Tape loading, unloading, and moving smoothly at proper speeds?

- Unusual system noises?

- Do tape packs appear smooth with no protruding layers after a rewind operation? Note: an occasional uneven pack can be due to an improperly slit tape.

- Tape winding tensions correct? If not, tape packs may appear irregular or loose.

22Most of these items must be performed by technical personnel such as Field Engineers; they include most of the replacements, adjustments, and trouble-shooting chores. However, a number of these items such as cleaning, can be performed by operating personnel who should also report apparent defects such as tape breaking, unusual noise, etc., to the person in charge. There are additional system checks which will be performed on-site by the Field Engineers including the proper reading and writing of the data on all tracks. A number of these suggestions can also be applied to cassette and cartridge drives. 
1.17 Computer Tape Transport Care

- Pneumatic system functioning properly? (i.e., check vacuum levels and pumps, vacuum and pressure points, valves, chamber sealing, etc.) Adjust or replace defective components.

- Belt tensions correct?

- Sudden increase in error rates? Report and investigate immediately. Check all potential tape damaging mechanisms described in sections $1.17-(1)$ and 1.2-(9). 


\section{Long-Term Storage - Care and Handling}

The purpose of the following sections is to describe the methods for controlling those factors which have a direct impact on the long-term archival storage of computer magnetic tapes and on the retrieval of their recorded information. The archival properties of the total systems and codes are not considered in this paper.

There is no unified or analytic discipline available at present with which the long-term properties of computer magnetic media and the stored data can be completely predicted. The available (and limited) information concerning the archival properties of flexible media such as computer tapes has been derived from two general areas:

(a) empirical observations, intuitive estimates drawn from experience, and surveys all based on large media populations [5] through [9], [25],[26], [27].

(b) extrapolation from small sample experiments and theoretical insights $[22],[28],[29],[30]$.

This section will present some of the information which is available for these two cases; i.e., the small and large sample computer tape populations. Each area will be presented individually. It should be understood that the information presented for the large sample case does not presently have the theoretical and experimental underpinnings of the small sample case, while the extrapolations from the small sample theories have not yet been proven to be effective when applied to a large, general tape population which contains a representative sample of the available media.

An underlying cause for the analytic complexity of this archival problem stems from the multivariable nature of the tape medium and its response to its handling, its systems, and its environment.

For example, it is of ten difficult to design a dynamic experiment which will lead to valid judgments about the probable end-of-life of the storage medium. Sometimes the dynamic test procedures will intrude their own characteristics into the results so that it is difficult to separate the cause and effect variables. An example of this type of problem appeared in an attempt to determine the effects on the "error-production" tendencies which existed between a tape transport system and reels of magnetic computer tape. It was found that the number of passes required for a single reel of tape to run to failure when shuttled among ten similar production transports was quite different from the number of passes that were required to run each of ten reels of the same kind of tape to failure on just one of these transports [31]. This difference was attributed to the machine differences rather than the tape differences. However, these transport differences were not evident during their operation in actual computer systems.

Another example in which the dynamic operating mode affects the end-of-life is as follows: There is a difference noted in the rate of data loss (drop-outs) if a magnetic computer tape is shuttled over short segments or over an equal number of passes over longer segments of the tape. This is caused by the variations in debris generation and distribution caused by the friction between the tape and (a) the mechanical guidance system, and (b) the recording/reproducing head. 
Although lifetime testing of a medium should be performed under conditions which are as similar as possible to the actual operating conditions, very often this is not a practical approach. This may be due to the expense or to the length of time which may be required for the test. Considerable care must be taken however when attempting accelerated lifetime studies because in many instances accelerated lifetime testing procedures yield results that are not valid because they are not truly representative of the actual environmental conditions in which the medium must function. As an example, by using very high temperatures it is possible to produce an observable breakdown of magnetic tape in a short length of time. However, in such a test, the researcher must be careful to remain below temperatures that can produce chemical phase transitions in the magnetic oxide particle material because the effects of heat are not cumulative for a tape. That is, during normal operations no amount of heat below the critical value can produce a phase transition (for example, a gamma to alpha transformation) in the oxide particles.

In a series of simulated and accelerated head-tape wear test procedures, it has been found that the relative wear results among a group of tapes reversed themselves as a function of the substitute metal which is used to simulate the head materials (see sec. 7.2).

Experimental evidence has shown that failure to extract the information from magnetic media is almost always attributable to the physical or chemical deterioration of the media rather than the volatility of the data. Data volatility is a function of the magnetic relaxation time of the recorded oxide particles assuming no intervention of undesirable energies such as high temperatures or unwanted magnetic fields [13]. Under ideal environmental conditions, the recorded data contents will outlast the physical and chemical structure of the tape materials at the present day particle sizes (volumes) and operating densities. In a controlled environment, it has been found that the recorded tape signal levels decrease $5 \%$ to $10 \%$ from their initial levels during the first 3 to 5 read cycles. At this point, the signals levels tend to stabilize so long as they are not subjected to read/write head demagnetizing fields.

An intuitive estimate for the improved modern computer tapes anticipates an effective lifetime of 20 years as compared to 10 to 12 years for tapes produced in the 1960s. The superior archival quality of the present day computer tapes is mostly due to improvements in the physical, chemical, and mechanical qualities of the tape rather than its magnetic properties. These stem from: (a) improved long wearing binder formulations which provide an efficient bond to the tape base (mylar) and resist the tendency to disintegrate or flake easily, (b) new surface calendering methods which have smoothed the tape surfaces by approximately $70 \%$ to $80 \%$ (i.e., to a present average surface roughnesses of $0.025 \mu \mathrm{m}(1 \mu \mathrm{in})$ to $0.15 \mu \mathrm{m}(6 \mu \mathrm{in}))$, (c) the application on many tapes of a new carbon based back coating material, and (d) improved manufacturing control of the physical parameters of the tape. All of these developments tend to reduce the production of tape debris and debris effects, as well as head wear. Loose debris particles can eventually lead to permanent tape damage when they are pressed into the surface by the large pack winding pressures during long-term storage. For example, it is possible to perform a successful read-after-write check on a computer tape prior to storage; however, the loose debris can permanently distort or "dimple" the tape and make that stored data irretrievable after long-term storage. 
The Archivist of the United States has established that "archival" is defined as "permanent-forever." However since most practical stored materials neither require nor possess such extreme capabilities, several other varied classifications have arisen. These are: medium term-up to approximately 10 years; long term--approximately 100 years; and expendable--used and discarded within a short time. Since it has been estimated that present-day computer magnetic tapes have a shelf life of approximately 20 years, it appears that the storage phase should be considered as medium term in length. However, the critical dynamic portion of the storage phase can extend the archival time well beyond the medium term. The dynamic storage phase is dedicated primarily to the long-term maintenance of the data contents through the process of scheduled testing and media replacement. Regular and master tapes can be recopied onto new reels ad infinitum since the physical medium has no intrinsic value as in the case of, say, historical documents. The replacement costs are partially offset when the data transfer is performed from a low density tape format to a newer high density format. This density increase reduces the number of required tapes and can release valuable vault space which is becoming extremely costly and scarce with the fast growth of computer tape archives.

\subsection{Long-Term Archival Storage - Large Population Cases}

The information in this section has been derived from many empirical and intuitive observations, operational experiences, and surveys based on large media populations. It has been organized in the following sequence [6],[25]:

\subsubsection{Preparation Phase}

(1) Quality of the Tapes that are Chosen for Long-Term Storage

(2) Physical Preparation of the Tapes Prior to Long-Term Storage

(3) Recommendations for Computer Tape Physical Preparation

(4) Preparation of the Data Prior to Long-Term Storage

(5) Recommendations for Data Preparation

\subsubsection{Storage Phase}

(1) Static Phase

(2) Recommendations (Static Phase)

(3) Dynamic Phase

(4) Recommendations (Dynamic Phase)

\subsubsection{Reactivation Phase}

\section{(1) Recommendations for the Reactivation Phase}

The preparation phase includes media selection and preconditioning processes. The storage phase is the phase which is most directly concerned with the actual archival properties of the stored media and particularly of their data contents and is considered in terms of controllable static and dynamic processes. Finally, the reactivation phase is concerned with the recovery of the stored data when the media are removed from the archives. 


\subsubsection{Preparation Phase}

(1) The Quality of the Tapes that are Chosen for Long-Term Storage

(a) The candidate tapes for operation at $8 \mathrm{bpmm}$ (200 bpi) and $32 \mathrm{bpmm}$ (800 bpi), NRZI, and $63 \mathrm{bpmm}$ (1600 bpi), PE, can be chosen to be in compliance with specifications which are described in documents such as the U.S. Interim Federal Specification W-T-0051C (GSA-FSS), March 1, 1975 , for $12.7 \mathrm{~mm}$ (0.50 in) computer magnetic tapes. 23 Compliance with these specifications assures the quality and the interchangeability of the computer tapes when they are used both internal to, or external to the originating computer system. Therefore, each tape will not be restricted to operate only with specific transports and electronic setups. Many available brands of standard quality computer tapes comply with this specification.

(b) The following tables 2. and 3. show some of the tests and examinations which were performed under the aforementioned specification W-T-0051C (GSA-FSS), March 1, 1975. It can be used as a guide to test procedures which can be instituted by media manufacturer and user organizations.

(c) An alternate source for use in making quality judgments relative to the chosen $12.7 \mathrm{~mm}$ ( $0.50 \mathrm{in})$ computer tape is the American National Standards Institute (ANSI) Standard entitled "Unrecorded Magnetic Tape (9-track, 800 CPI, NRZI; 1600 CPI, PE; 6250 CPI, GCR) (ANSI Docum ent: X3.40-1976). ANSI has also developed a series of standards for other computer storage media such as flexible disks, cassettes, and cartridges. Note that unrecorded standards are required for testing information similar in detail to the GSA-FSS specification.

(d) There are computer tapes which are described by their manufacturers as "permanent," "archival tapes," etc. However since the ICST Computer Storage Media Group has not performed any in-house experimentation with such extended life magnetic computer tapes, the following statements are not to be construed as an endorsement of these media. There are computer tapes on the market which are claimed to have potential lifetimes of 20 years and a capacity for millions of passes. Typically, their long-life characteristics are based upon extrapolations from accelerated test procedures. It has been reported that some of these extended life tapes have very hard binders which can cause rapid head wear. In addition, it has been found that the final error production tendencies of some long-life, hard binder tapes eventually approached that of the standard tapes [7].

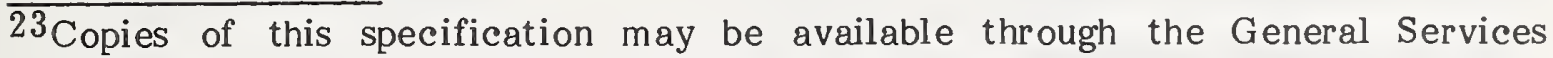
Administration, Automated Data Telecommunication Services. The GSA-Magnetic Surfaces Laboratory which performed this specification testing was terminated in February 1982. 
TABLE 2. COMPUTER TAPE TESTS, MEASUREMENTS, AND EXAMINATIONS

1. Tape length

2. Tape width

3. Tape thickness

4. "E" value

5. Photoreflective markers

6. Marker reflectivity

7. Yield strength

8. Elongation under stress

9. Longitudinal curvature

10. Abrasivity

11. Cupping

12. Stiffness

13. Splices

14. Output signal level

15. Surf ace unif ormity defects

16. Ease of erasure

17. Dynamic skew

18. Start time

19. Ten-foot wear

20. Compatibility

21. Wear deposits

22. Environmental wear

23. Electrical resistance
24. Tape reel dimensions

25. Flange label area

26. Write enable ring

27. Tape reel environmental stability

28. Moment of inertia

29. Loss of recorded information 
1. Reels:

Appearance

Identification and labeling

Reel color

Write enable ring

2. Tape:

Wind

Wound pile (pack) envelope

Appearance

Construction

3. Reflective markers:

4. Reel containers:

5. Condition as received:
Voids, nicks, and other surface imperfections which do not fall within an area of a circle $1.6 \mathrm{~mm}$ (0.063 in) in diameter; scratches which exceed $12.7 \mathrm{~mm}$ (0.50 in) in length.

Not as specified?

Not as specified?

Missing; improper fit.

Not as specified?

Tape pile (pack) not smoothly wound; loose, with visible folds, buckling, cinching, spoking, or gaps between the tape layers. Protruding tape edges, tape layers, or groups of layers. Plane of the tape pile not perpendicular to the reel axis.

Tape surfaces not clean; presence of dirt, dust, lint, fuzz, or other foreign matter. Presence of blemishes, holes, tears, creases, or wrinkles, split or ragged edges. Presence of adhesive substance.

Tape does not unroll evenly and uniformly; tape sticks.

Missing; incorrect placement; wrinkled; improper method of application.

Not as specified?

Improper packaging; improper marking; damaged contents. 
There is continuing research by media manufacturers towards producing a premium or superior line of computer tapes whose lifetimes and adherence to required interchange parameters are better than those which are achieved with standard brands. There are expensive premium tapes with a lifetime guarantee; these tapes will be replaced free of charge if any errors ever develop [12(b)]. Unfortunately, the replacement cost of a tape is minor when compared to the cost for reconstructing permanent read errors which have developed in archives. It should be noted that tapes which are designated for long-term archival purposes do not necessarily require the capability for withstanding a large number of passes. In fact, the hardness or sof tness of a tape binder is not necessarily an indicator of the durability of the tape [18]. Instead they require physical/chemical stability over long periods of time and proper care and handling techniques in archives. The effect of the archival handling methods on tape life can be monitored through the scheduled use of error logging routines which periodically read, detect, and count accumulated tape errors from the stored files in archives. This can yield useful statistical information relative to pending problems and possible corrections prior to major losses. At the present time, however, the long-life computer tape user position should be one of caution until sufficient long-term evidence has been presented on behalf of particular brands of archival tape which are made available in the marketplace.

(e) Computer magnetic tapes which were produced prior to 1970 should not be chosen for long-term storage even if they have never been used previously since it has been found that they have a greater tendency to develop permanent errors as a function of time in storage than tapes which were developed and produced in the $1970 \mathrm{~s}$ and 1980s. It was found for the pre-1970 tapes that the buildup and accumulation of tape errors in storage increased approximately as an exponential function of time and that errors developed even in good storage environments [6]. This error growth is particularly evident in the older tapes which have been recorded at 32 bpmm (800 bpi) (NRZI).

(f) In order to achieve greater security against the loss of data due to tape deterioration in the primary or backup storage location, do not use tapes from the same manufacturer's lot for both the archival tapes and the backup (duplicate) tapes.

(2) Physical Preparation of the Computer Tapes Prior to Long-Term Storage

Factors which influence the physical preparation of the tapes prior to storage are:

(a) New tapes are sometimes contaminated with debris which eventually can become a source of read/write errors. Some of this debris can originate in the tape slitting process.

(b) Tape life is affected significantly by the condition of the initial tape drive which records the data for storage. For example, it can introduce debris into the tape windings; it can also produce an unevenly wound tape pack. This uneveness can produce laterally displaced windings whose edges can be damaged by operator mishandling of the reel flanges (see sec. 1.2-(1)). 
(c) The tape rewind tension after recording has a significant affect on the long-term storage quality of the tape. The tape drive should not introduce large tension variations when rewinding the tape prior to storage. Too small or too large a tension can cause eventual tape problems. Excessive tape winding pressures and humidity and temperature swings in storage can produce permanent distortions or damage to the tape, while very low winding pressures can produce layer-to-layer slipping and creasing or "cinching" in the tape pack upon acceleration which can lead to serious data losses (see secs. 7.3 and 7.4).

\section{(3) Recommendations for Computer Tape Physical Preparations:}

(a) All tapes, including new tapes, which are selected as candidates for long-term archival storage applications should be cleaned and tested (evaluated) at the anticipated recording density. Certification and manual error removal is an optional activity (see secs. 3.2.1 to 3.2.3).

(b) The cleaning and evaluation should be performed within six months of application prior to recording and storage.

(c) A maximum of five write skip errors should be allowed on any candidate tape prior to long-term storage; none of these errors should exist on the first $30.5 \mathrm{~m}(100 \mathrm{ft})$ of tape. No permanent errors are permitted (see sec. 3.1). Scratch or rehabilitated tapes are not recommended for archival storage.

(d) The candidate tapes should have less than 50 full passes but more than four passes. New tapes should be exercised for the required minimum of four full passes prior to recording.

(e) Prior to final storage, after cleaning and evaluating, while awaiting the recording of the archival data, the tapes should be given a full length pass at normal speeds, no high speed rewind should be performed. The winding should be performed in a constant tension or programmed rewind mode at approximately $1.7 \mathrm{~N}$ (6 ozf) to $2.2 \mathrm{~N}$ (8 ozf) per $12.7 \mathrm{~mm}(0.50 \mathrm{in})$ width of tape (see sec. 7.4).

(f) Fasten all tape leaders with a soft sponge, rubber grommet, or vinyl strip.

(g) Make certain that the reel is properly labelled with a self-adhesive label. Write with a ballpoint pen or typewriter on the label. Do not use a graphite pencil or any water soluble marker. A label may not be necessary if a Tape Management System is used (for example, see sec. 4.2).

(h) Place each tape into a fully enclosed, clean transparent canister. Make certain that the canister is fully and tightly latched. Wrap-around collars should not be used with tape reels whose flanges have windows or apertures (see sec. $1.3-(6))$.

(i) As an option, seal the canister in a polyethylene bag. This reduces the evaporation loss of components from the tape coating. 
(j) Store tape in an upright (on edge) position in vault. The canister will support the reel by its hub which is its strongest member. For long-term archival storage, avoid devices which support the tapes by their flanges rather than by their hubs.

\section{(4) Preparation of the Data Prior to Long-Term Storage:}

Some factors which influence the preparation of the data prior to storage are:

(a) Tapes which are recorded with misaligned read/write transducers may be difficult to read back on any transport other than the originating unit.

(b) Tape transports which have dirty, worn, or misadjusted guidance systems can damage the tape, cause skew effects, and add debris to the tape. These defects will eventually cause the loss of data in storage.

(c) It has been reported that computer tapes which had been recorded at $32 \mathrm{bpmm}$ (800 bpi) using the non-return-to-zero (NRZI) mode of recording tended to develop more errors in storage than tapes which were recorded using phase encoded (PE) methods [8], [9]. It has been recommended that either a $63 \mathrm{bpmm}(1600 \mathrm{bpi}) \mathrm{PE}$ method or an NRZI method at a lower information density such as $8 \mathrm{bpmm}$ (200 bpi) should be used for archival storage purposes. Although relatively new, it appears that the high density 246 bpmm (6250 bpi) computer tapes have good archival qualities. However, there are both favorable and unfavorable factors:

(1) Favorable: The overall handling of the computer tape by the typical $246 \mathrm{bpmm}$ (6250 bpi) drive tends to be very gentle. For example, the oxide coated side of the tape generally does not rub or contact against any transport or guidance surface other than the read/write head, erase head, and cleaner assembly. There are usually very small and well controlled winding tension variations on the present day $246 \mathrm{bpmm}$ (6250 bpi) drives [20].

(2) Unfavorable: Since $246 \mathrm{bpmm}$ (6250 bpi) has a maximum recording density of $356 \mathrm{bpmm}$ (9042 bpi), the wavelengths of these signals are very short. Hence, it requires a very small head-to-tape spacing to create a significant signal drop-out loss (see sec. 8.1-eg(4)). In other words, debris particles whose size would cause no problems at $63 \mathrm{bpmm}$ (1600 bpi) can cause data losses at $356 \mathrm{bpmm}$ (9042 bpi).

(d) Another experimental finding of interest is that there are regions along the length of many computer tapes that appear to be more susceptible than other regions to developing data losses while in storage. On a typical $731 \mathrm{~m}(2400 \mathrm{ft})$ length computer tape, these undesirable regions are from the beginning of the tape to the $100 \mathrm{~m}(328 \mathrm{ft})$ point, from $300 \mathrm{~m}(984 \mathrm{ft})$ to $400 \mathrm{~m}(1312 \mathrm{ft})$ and from $600 \mathrm{~m}(1968 \mathrm{ft})$ to the end of the tape [26].

It was noted that if a recording strategy could be devised which avoided these regions, then there could be a significant increase in the useful storage life of the data on the tape medium. In this study, a tape was rejected if more than one parity error was detected over any $300 \mathrm{~m}(984 \mathrm{ft})$ 
of the tape. It was also felt that an ef fective strategy might be developed which was based upon the statistically derived observation that there was an optimal block length which minimizes tape errors while realizing efficient packing of the data. The block lengths however are usually constrained by the system requirements and a practical application of this concept is difficult to achieve.

(e) It has been noted that tapes which are recorded and read over only a small portion of their total lengths display a greater tendency towards cinching. This is due to irregular variations in the tape pack tension caused by the partial wind-rewind processes.

(5) Recommendations for the Preparation of Archival Data:

(a) Make certain that the transports and transducers are properly cleaned and adjusted prior to recording.

(b) Check the read/write and tape motion operations of each transport before commencing with the recording of the archival tapes. Do this with a known clean error-free tape which can then be used to perform a read-only pass on all of the transports. This will check their relative alignments.

(c) Record the archival tapes and then remove the write enable rings in order to protect the tapes against erasure. (Note that this may not be required with a Tape Management System such as TMS-II (see sec. 4.2).)

(d) Perform a "read-only" pass on each tape in order to verify that an error-free recording has been made prior to storage.

(e) When performing this verification, the tape should always be given a full length pass to the end-of-tape marker (EOT), and then rewound at a normal speed only. The winding should be performed in a constant tension or programmed rewind mode (see sec. 7.4). A normal tension value of approximately $1.7 \mathrm{~N}$ (6 ozf) to $2.22 \mathrm{~N}$ ( 8 ozf) per $12.7 \mathrm{~mm}(0.50 \mathrm{in})$ width of tape is most frequently suggested.

(f) Avoid the use of $32 \mathrm{bpmm}(800 \mathrm{bpi}$ ) (NRZI) recording for tapes which are to be placed into long-term storage.

(g) Important option: if possible, immediately record the backup copies of all vital tapes and move them without delay to the alternate location. Perform all steps in item (5). The backup copies should be recorded either in, or as close to, the primary archival storage area as possible. Do this in order to avoid environmental or physical damage to the vital tapes when they are in transit to an external duplicating facility.

\subsubsection{Storage Phase}

The storage program phase is the one most directly associated with the long-term maintenance of the recorded media and the systems. Since it has been estimated that present day computer magnetic tapes have a shelf life of approximately 20 years, it appears that the storage phase can be considered as a medium life term program as previously defined. 
The storage program consists of a static phase and a dynamic phase. The static phase deals with the control of the ambient storage environment and the physical housing of the magnetic media. The dynamic or active portion of the storage program consists of measures which can be undertaken, of ten on a scheduled basis, to prevent, to seek out, and to circumvent the loss of stored data due to the physical distortion or chemical deterioration of the media. The dynamic portion of the storage phase can extend the life of the data well beyond the medium term if it is deemed feasible and necessary.

\section{(1) Static Phase}

The static measures consist of maintaining a proper temperature, humidity, cleanness, and security environment for the stored tapes. A good storage environment is one which approximates the characteristics of a tape measurement laboratory (for example, see sec. 5.2-(5)). Although the very high class clean room conditions which are produced, for example, with laminar air flow systems through HEPA filters (see sec. 5.1) are not needed, positive internal air pressure systems in the storage areas are highly recommended. This will prevent the intrusion of external dust into these areas. Whenever possible, the temperature and humidity of the storage area should be close to that of the operating computer environment in order to avoid subjecting the tapes to large ambient changes when they are transferred from the vault to the computer installation. If possible, the vault temperature and humidity should be within $+2.8^{\circ} \mathrm{C}\left(+5^{\circ} \mathrm{F}\right)$ and $+5 \% \mathrm{RH}$ of the computer room's T-H environment.

There are commercial organizations available that provide secure storage vault services for computer magnetic tapes, microfilms, and paper. They are of ten located underground in order to protect the stored materials against explosion. They are held at a constant temperature and humidity and typically employ electronically filtered air systems. The use of these commercial repositories 24 is very valuable if a redundant storage approach is taken, i.e., duplicate tapes are made for either all or part of the tape library and stored in various locations as a precautionary measure.

It has been found that a magnetic field supplies the only kind of energy that can cause undetected data destruction without any accompanying physical distortion or damage to the magnetic storage media (see secs. 1.11-1.12). It has been determined experimentally that there is no need to shield the stored data against $x$-rays, high voltage fields, nuclear radiation, high frequency fields, or light energy (see secs. 1.9-1.10). Note that a spacing of $76 \mathrm{~mm}$ ( $3.0 \mathrm{in})$ is sufficient to protect the recorded media against magnetic fields which are far more intense than are ever found in a normal environment, or that can be produced by a concealable permanent m agnet.

$24 \mathrm{Commercial} \mathrm{storage} \mathrm{facilities} \mathrm{for} \mathrm{computer} \mathrm{magnetic} \mathrm{media} \mathrm{can} \mathrm{of} \mathrm{ten} \mathrm{be} \mathrm{located}$ through the Yellow pages of the telephone directory (see sec. 1.2-(33)). 
Finally, security measures should be undertaken to protect the tape vault against unwarranted intrusion or catastrophic damage. This is done with monitoring devices such as magnetometers and properly insulated vault construction which can protect the tapes against fire hazards. Fire has been found to be the greatest threat to the magnetic tape repository.

(2) Recommendations (Static Phase)

(a) Maintain vault temperatures in the range of approximately $15.6^{\circ} \mathrm{C}$ $\left(60^{\circ} \mathrm{F}\right)$ to $26.7^{\circ} \mathrm{C}\left(80^{\circ} \mathrm{F}\right) .25$

(b) Maintain a vault humidity range of from $40 \%$ to $60 \% .25$

(c) Maintain the vault temperature and humidity within $+2.80^{\circ} \mathrm{C}(+5 \mathrm{OF})$ and $\pm 5 \% \mathrm{RH}$ of the $\mathrm{T}-\mathrm{H}$ of the computer installation.

(d) Employ positive internal air pressure systems to reduce dust contamination in the vault.

(e) Use commercial or other alternate vault systems for redundant tape storage of vital tapes if possible (see sec. 1.2-(33)).

(f) Store tapes in canisters in an upright (vertical) position. Seal the canisters in polyethylene envelopes.

(g) Maintain vault security against entry by unauthorized personnel (see sec. $1.12-(4))$.

(h) Use magnetometer detection for controlled entry of permanent magnets into the vault area. See section 1.12 for methods for protecting the stored media against magnetic fields.

(i) Observe all of the fire safety recommendations listed in section 1.7. For master and vital tapes, consider the specialized vaults recommended in section $1.7-(5)$.

(j) Consider the potential for vault area flooding and fire fighting when designing the installation (see sec. $1.8-(8)$ ).

(k) Carbon dioxide $\left(\mathrm{CO}_{2}\right)$, Halon, and water are permissible for fire extinguishing. Large plastic sheets should be kept on hand in order to cover the tape racks and reduce water damage in case of a fire or an inadvertent water sprinkler discharge.

25 Note that these temperature-humidity conditions for long-term archival storage are more stringent than those given in section 1.4-(1). These recommendations are applicable to computer tapes which were produced prior to 1972 and therefore require closer tolerances. 
The dynamic portion of the storage program consists of scheduled active measures which are instituted in order to improve the chance of survival of stored magnetic computer tapes and their contents. These measures include tape cleaning, tape rewinding, physical inspection, recopying of key tapes, and scheduled reading of sample tapes from all of the tape files.

It has been found that if tapes are exercised by being wound and rewound on an annual or semiannual basis, then many incipient tape errors can be delayed or circumvented. The rewinding process which should be performed at normal operating tape speeds, will remove some of the stresses which have built up in the tape pack during the storage interval (see sec. 7.4).

Temporary errors can often be removed by cleaning and rewinding the tape, while permanent errors, which are caused by defects such as fused surface debris, missing oxide, deep dimples, scratches and creases, are not removable (see definitions in sec. 3.1). It has been found that temporary tape errors tend to become permanent if they exist in storage for a long time. Usually a small sample drawn from a large tape file is representative of the tapes in that same file. It is recommended that a random sample of tapes from each file should be read on an annual basis and checked for permanent errors. If a number of permanent errors have developed in the sample tapes, it is necessary to read through the entire file from which they were drawn and recopy or replace all defective tapes. Computer installation managers will sometimes recopy important tapes on a semiannual or annual basis as a normal routine [8]. Even if there are no permanent errors found on a stored master or vital tape, it may still be feasible to recopy the data onto a newer state-of-the-art tape and to rehabilitate the original tape for future use in less critical scratch applications than long-term archival storage.

As an optional measure, in lieu of, or in conjunction with the random sampling approach, place a number of control tapes into storage along with each major group of archival tapes. They should emanate from the same tape lots and be recorded and wound on the same transports as the regular stored tapes. The number of control tapes should also be based on the size of the individual archival groupings and importance of the data. The condition of these tapes and their data can be examined on a regular basis and used to monitor the condition of the archival tapes.

The dynamic program phase recommendations should be reexamined and revised at intervals in order to accommodate the continuing technological changes and improvements in magnetic media, their associated equipment, and recording methods. These factors may eventually make less stringent archival handling methods a possibility.

The institution of a full scale dynamic handling program in an organization with a large tape archive can be very expensive since it may require an organizational unit with additional personnel and equipment for performing the operations. As an efficiency measure, it may be suggested that the 
stored media can be classified as a function of their relative importance and that the scheduled procedures can then be based upon these classifications. That is, the frequency of reading, testing, cleaning, exercising, and recopying will depend upon the value category which is assigned to the tape data. The organization and scheduling procedures particularly in the case of large archives, can be handled very effectively under the control of a Tape Management System (see secs. 4.1 to 4.3). Testing may be performed non-destructively with the software system described in section 4.4.

(4) Recommendations (Dynamic Phase)

(a) If possible, maintain the necessary equipment and perform the scheduled care and handling procedures directly in the archive environment. This minimizes the physical hazards and the temperature and humidity variations which the tapes can be subjected to in transit. If a tape has been transported from an operational area to an archive, permit it to relax for 24 hours in the new environment before it is used (alternately, use sec. 1.5-(4)). Perform step (c) before replacing the tape into its normal rack position in the vault.

(b) Maintain all of the required equipment such as magnetic tape transports and cleaners (see sec. 3.2) in a clean and properly adjusted condition. Check for points of wear in the tape head and guidance system which can cause tape damage (see sec. 1.17). Clean the entire system immediately after a defective tape has been run, especially if it has shed coating materials. Make certain that the transport components are not defective and have not become magnetized; degauss regularly (see sec. 1.12-(5)).

(c) Exercise each stored tape with a full forward and reverse pass at normal speeds on an annual or semiannual scheduled basis. Use a constant tension or programmed tension wind at normal tape speeds. It is advantageous to perform this exercise on a tape cleaner with good winding characteristics since it will accomplish a dual function; i.e., cleaning plus exercising.

(d) Read at least one tape from each small tape file and a $3 \%$ random sample from each large tape file on an annual basis. If any permanent read errors have developed in these sample tapes, then the entire file is suspect and should be rechecked. The data on any tape which has developed permanent read errors should be recopied onto a new reel of tape; these procedures are absolutely necessary for master tapes. A recheck should also be performed on those tapes which emanate from the same manufactured lot as the defective tape. If possible, carefully inspect the condition of the machine or machines which recorded these defective tapes since they may have caused the error problems. Backup files are very valuable for recovering data lost due to permanent read errors.

(e) Set up a schedule for checking the condition of the media and the data on the optional control tapes described in sections 2.1.2-(3) and 
1.2-(28). This will give an indication of the condition of the associated ar chival groups.

(f)' Tapes which have developed temporary errors (see sec. 3.1) should be cleaned and reread. Consider recopying tapes on which a large number of temporary errors have developed in storage since this could be a sign of coating deterioration. Other tapes from the same lot should be rechecked.

(g) As an option, recopy master tapes on an annual basis regardless of their condition [8]. Regular tapes may be recopied after a longer period of time in archives. It has been suggested (optimistically) that recopying should be done at least once every ten years for the present day, state-of-the-art computer tapes.

(h) Inspect the tapes for irregular windings, creases, warped edges, discoloration, or other physical anomalies (see sec. 1.2-(21)). Clean and read those tapes which are suspect and recopy them if necessary. If there has been a serious loss of environmental controls during long-term storage, an extensive visual check and rereading should be performed on all vital tapes.

\subsubsection{Reactivation Phase}

When the tapes which have been in long-term storage are brought out to the computer installation from the vault, they should be permitted to adjust themselves to the ambient temperature and humidity of that environment for approximately 24 hours before being placed into operation. Alternately apply section 1.5-(4) if accurate $\mathrm{T}-\mathrm{H}$ data are available. The operating environments at the archives and the computer installation should be maintained as clean areas by using positive internal air pressures and observing all other clean operating guidelines (see sec. 1.3).

It is possible that data losses will occur on reactivated media even though proper care and handling procedures were observed during the preparation and storage phases (see secs. 2.1.1 and 2.1.2). These losses can be caused by improper random sampling processes (see sec. 2.1.2-(4)) or possibly by a an environmental failure, such as an accidental air conditioning outage which occurred at some time during the storage phase. They may also be caused by a defective tape coating, possibly due to prior hydrolysis (see secs. 2.2.1 and 7.1) which led to a breakdown in the coating chemistry during storage.

It is also possible that the operational characteristics of the data preparation tape transports are sufficiently different from the reactivating transports that marginal operations result. For example, differences in head wrap angle, tape tension, and guidance systems may produce data problems. It is an obvious advantage if the same transports are used for both data preparation and reactivation.

There are some corrective measures which can be undertaken in the event that data loss problems are found to have developed during the reactivation phase. For example, it was noted previously that tapes which are recorded at $32 \mathrm{bpmm}$ (800 bpi) in the NRZI mode tend to lose data easily. Cold flow distortion and skewing of the 
tape is believed to be the principal cause for data loss at this density. Sometimes the skewed tape can be sufficiently straightened by rewinding it at normal speeds, for say ten times, and then relaxing the tape for one or two days before finally winding and rewinding it several more times on the transport which will be used to read the tape. This same procedure can be initiated before reading a tape if it is noted that the tape windings appear to be irregular with some tape edges protruding from the pack.

There is a wide range of electronic and mechanical transport adjustments which can be performed by technical personnel which will recover marginal tape information. Quite of ten a tape which is difficult to read on one transport can be read easily on another. Some tape drives which have the capability of reading backwards can of ten read a balky computer tape which cannot be read in the forward direction. The reactivating tape transports should be degaussed on a scheduled basis (see sec. $1.12-(5))$.

It should be noted that the reactivation phase recommendations are particularly important for computer tapes which are stored for one year or longer.

\section{(1) Recommendations for the Reactivation Phase}

(a) Relax the reactivated tapes in the computer environment for 24 hours before attempting the read process. If possible, the computer room temperature and humidity should be maintained within $+2.8^{\circ} \mathrm{C}\left(+5^{\circ} \mathrm{F}\right)$ and $+5 \% \mathrm{RH}$ of the vault's $\mathrm{T}-\mathrm{H}$ environment.

(b) If read difficulties are encountered, perform a visual check of the reactivated tape (see sec. 1.2-(21)). If layers of tape appear irregular, folded, or separated or if there are protrusions of individual tape layers from the pack edges, then wind and rewind the tape twice over its entire length at normal (slow) speeds. This can be done on a tape transport or cleaner/winder unit. Now relax the tape for at least one more day, then wind and rewind the tape several times before attempting the read process.

(c) Maintain all of the magnetic tape transports and cleaner/winders in a clean and properly adjusted condition. Check for points of wear in the tape head and guidance systems which can cause tape damage (see sec. 1.17).

(d) Clean the entire tape transport immediately af ter a defective tape has been run, especially if it has shed coating materials and other debris. This debris can propagate to the following tapes and cause additional error problems (see sec. 1.3-(12) and table 1.).

(e) Immediately clean and attempt to recopy the reactivated tape which has produced the debris in (d), particularly if it was caused by coating breakdown or deterioration.

(f) When reactivating tapes, avoid using tape transports with known marginal or poor performance histories. 
(g) Reactivated tapes which display skew losses due to longitudinal distortion can sometimes be corrected by perfor ming step (b).

(h) If the read problems persist, then if possible, attempt to read and recopy the problem tapes on the transports which originally recorded the data. If unsuccessful, try several other tape transports for reading the balky tapes.

(i) Technical personnel can of ten recover marginal tape data through electronic and mechanical adjustments of the tape transports. The data may have decreased in signal level due to magnetized components on a tape transport on which the tapes have been run or due to exposure to stray magnetic fields (see sec. 1.12).

(j) Some tape transports (for example, 63 bpmm (1600 bpi), phase-encoded units) are capable of reading data in the reverse direction; this mode of operation can of ten be tried in order to read a balky tape which has failed in the forward read mode.

(k) Since data reconstruction processes from a faulty tape can be costly, it is much more efficient to produce a new tape copy from a backup tape file, if one exists.

\subsection{Long-Term Archival Storage - Small Sample Population Case}

This section is based on a comprehensive study [22] dealing with the archival properties of magnetic tape storage media such as instrumentation and computer tapes. The study presents the results of an experimental and theoretical program which was performed with a small number of media and is the most extensive publication of this type which has been found to date.

The degree of confidence with which these results can be extrapolated to include large, variable source, computer tape populations is presently unknown. However, some of the recommendations and methods offered by this study are sufficiently unique to warrant consideration for all cases.

This small sample population section is presented in the following sequence:

2.2.1 Background Theory for S mall Sample Population Recommendations

\subsubsection{Preparation Phase}

(1) Quality of Tapes That are Chosen

(2) Physical Preparation Prior to Long-Ter m Storage

\subsubsection{Storage Phase}

(1) Static Recommendations

(2) Dynamic Recommendations

\subsubsection{Reactivation Phase Recommendations}




\subsubsection{Background Theory for Small Sample Case Recommendations}

(a) All of the recommendations in this small sample study [22] are based on magnetic tapes which utilize the prevailing polyurethane binder systems, polyethylene terephthalate (i.e., mylar) base films, and gamma ferric oxide $\left(\gamma-\mathrm{Fe}_{2} \mathrm{O}_{3}\right)$ magnetic particles. The choice of the polyurethane binders is based on their tensile strength, toughness, abrasion resistance, and adhesion properties.

The reaction of magnetic particles such as gamma ferric oxide $\left(\gamma-\mathrm{Fe}_{2} \mathrm{O}_{3}\right)$, chromium dioxide $\left(\mathrm{Cr}_{2}\right)$, iron particles $(\mathrm{Fe})$, and various cobalt-doped gamma ferric oxide particle structures to different temperatures and humidities was studied. The following conclusions were reached:

(1) A magnetic tape composed of $\gamma-\mathrm{Fe}_{2} 0_{3}$ does not exhibit long-term demagnetization or print-through when used in digital recording applications of up to $1300 \mathrm{bpmm}(33,000 \mathrm{bpi})$ and stored in nominal environments.

(2) The archivability of magnetic tape employing $\gamma-\mathrm{Fe}_{2} \mathrm{O}_{3}$ particles is reasonably guaranteed at $20^{\circ} \mathrm{C}(68 \mathrm{OF})$ or cooler temperatures, and $50 \% \mathrm{RH}$. These conclusions are extremely important since $\gamma-\mathrm{Fe}_{2} 0_{3}$ is by far the most widely used particle for computer magnetic tapes. It was found that chromium dioxide and $\mathrm{Co}-\gamma-\mathrm{Fe}_{2} \mathrm{O}_{3}$ might not be as suitable for archival purposes due to their sensitivity to environmental conditions.

(3) The study noted that the effects of time, temperature, and humidity on the magnetically recorded signal (on the $\gamma-\mathrm{Fe}_{2} \mathrm{O}_{3}$ ) are negligible even though their effects on the binders and base films may be severe. It was concluded that the tape recording format may be chosen for optimum storage density independently of archival environmental considerations.

(b) A hydrolysis study (which is a study of chemical reaction of water with the polymer material) was made with tapes employing polyurethane binders. The effects of hydrolytic action on tape binders was studied in-depth [28] [29], [30] and the rate of hydrolysis was found to depend upon the binder constituents. In hydrolytic action, the combining of the atmospheric water vapor with the coating esters produces carbolic acid and alcohol. This ester reaction causes binder weakening and the ensuing gumminess and breakdown of the tape coating. Experiments have shown that the hydrolysis process is reversible. This indicated the possibility of tape binder rejuvenation; i.e., a binder which had been deteriorated by hydrolysis, could be restored to a normal condition by storage in a cool and dry environment. For example, the hydrolysis equations showed that a tape which is stored at $27^{\circ} \mathrm{C}\left(80^{\circ} \mathrm{F}\right)$ and $80 \% \mathrm{RH}$ will suffer a $16 \%$ binder consumption in less than one year in this $\mathrm{T}-\mathrm{H}$ environment. The same equations show that it is a reversible process and that it will require approximately 1.5 years for the binder to return to its original state. This is considered to be an impractical length of time to wait for binder recovery.

The zero hydrolysis curve in figure 3. (cour tesy [22]) represents those conditions for which a binder that has an initial degradation of $6.7 \%$ will not be further degraded under any $\mathrm{T}-\mathrm{H}$ conditions along that curve. However, for values of 
temperature and humidity below the zero hydrolysis curve in (Zone 1), the hydrolysis rate is negative; i.e., presumably here the binder will be rejuvenating as ester bonds are regenerating. In Zones 2 and 3, the hydrolysis process continues to consume the binder materials, leading to eventual binder breakdown. Zone 3 must be avoided as indicated in figure 3 .

A key conclusion and recommendation derived from this chart is: for tapes stored at $18{ }^{\circ} \mathrm{C}\left(65^{\circ} \mathrm{F}\right)$ and $40 \% \mathrm{RH}$, the polyurethane binder consumption is zero; and most importantly this is also a fairly comfortable environment for the handling personnel.

Additional study will be required in order to determine the relationship of binder degradation due to hydrolysis with an associated increase in data error rates. The effects of the recording methods, the detection scheme, and the error correction techniques must also be considered when analyzing the error rate processes.

(c) As noted in sections 7.3 and 7.4 due to their ereep and stress relaxation characteristics, viscoelastic materials which are wound under tension require occasional rewinding in order to minimize deleterious effects such as tape cinching. The required rewind frequency has been found to be a multivariabled function of the storage time, the temperature, the magnitude and duration of variations in temperature and humidity, winding tensions and tape speeds, reel size, interlayer friction coefficients, and the reel acceleration during rewind. It is noted that if a $50 \%$ pressure reduction in the tape pack due to creep and stress relaxation is an indicator of the need to rewind the tape, then an approximate period of six months between rewinds is deduced as the proper scheduling. It also notes, however, that tapes which are stored under well controlled temperature and humidity conditions could be stored for much longer periods without rewinding. In fact, a period between rewinds of 3.5 years is recommended provided that the tapes have been maintained in a controlled storage environment as follows (see rectangle in fig. 3 ):

Temperatures: $17^{\circ} \mathrm{C}\left(62^{\circ} \mathrm{F}\right)$ to $20^{\circ} \mathrm{C}\left(68^{\circ} \mathrm{F}\right)$

Relative Humidity: $35 \%$ to $45 \%$

If the environment cannot be maintained in these ranges, then the conventional semiannual or annual rewind schedule is recommended. Also if large environmental changes have occurred, then even more frequent rewinds may be advisable.

(d) A significant portion of [22] was devoted to the study of the physical and mechanical aspects, both theoretical and experimental, of the tape reel under various winding tensions and pressures. It was decided that if there were a method for measuring the amount of tape pack winding pressures which exists at any time in a stored reel, then an economical and ef ficient rewind frequency schedule could be developed. Several different approaches to the pressure measurement were considered including: the correlation of the speed of wave propagation through the reel as a function of winding tightness, the measurement of the reel hub deformation with an at tached strain gauge, and finally the insertion of friction tabs into the reel during winding. This last technique was considered as the most promising approach and a considerable amount of testing and experimentation was performed with these friction tabs. 


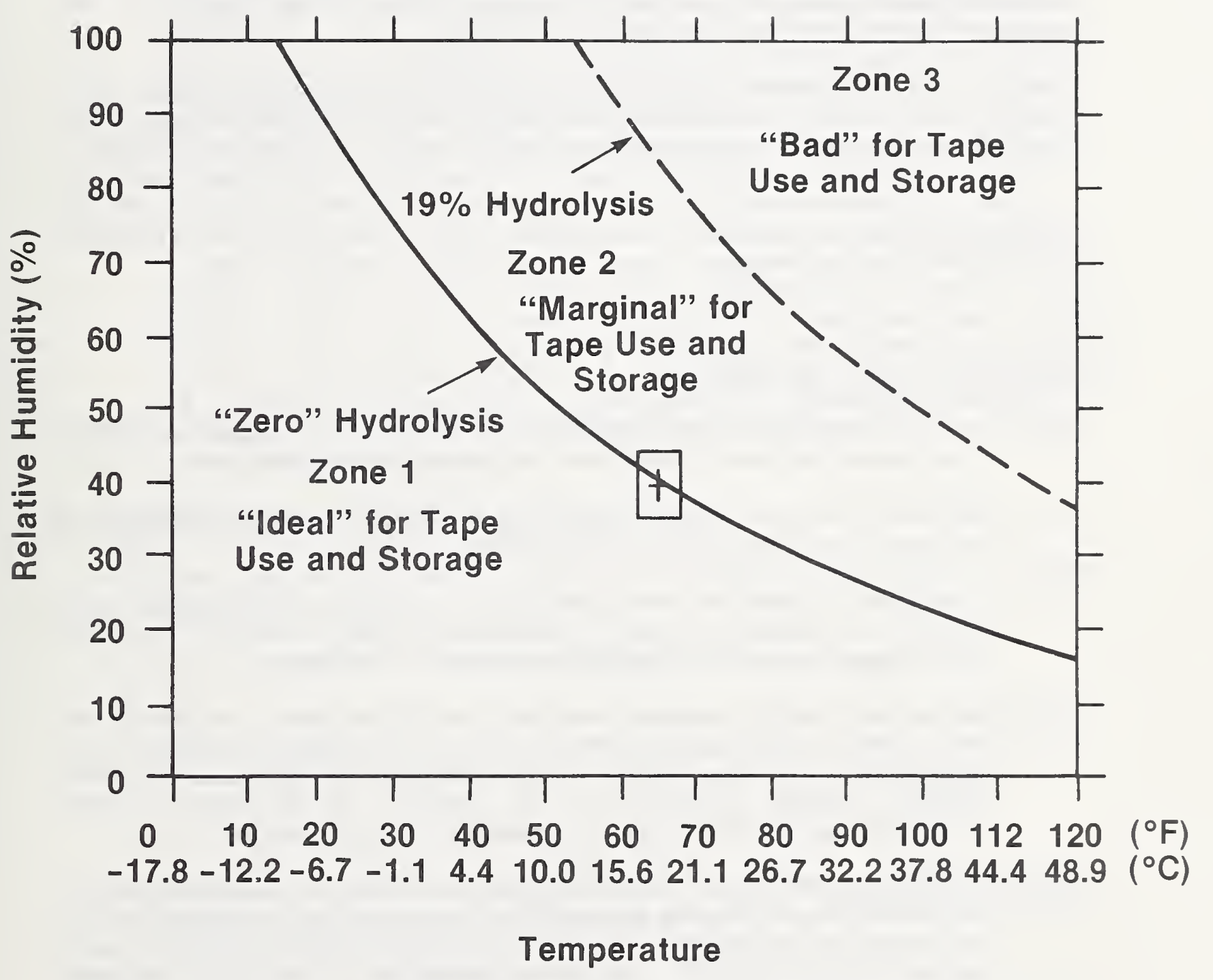

Fig. 3 
The basic process consists of measuring the dislodging pull force required to extract these friction tabs from the reel winding using spring scales. Approximately, one dozen tabs are inserted into each reel in a spiral arrangement. The tabs are typically $.025 \mathrm{~mm}(.001 \mathrm{in})$ thick, $6.35 \mathrm{~mm}(0.25 \mathrm{in})$ wide and $50.8 \mathrm{~mm}(2.0$ in) long. Tests were performed at different winding torques, decelerations, winding speeds, and temperatures. Interestingly, it was found that for constant speed windings at different torques, that a $20 \%$ reduction in winding tension may be responsible for a $50 \%$ reduction in tab pull forces. It was theorized that this relationship was caused by interlayer, trapped air which developed during winding. It was also found that there was a specific tab pull force value below which the tape reel would display interlayer slippage (and cinching) upon deceleration. The appearance of this pull force value can be used as an indicator of the need for tape rewinding (retensioning) due to the reduction in the layer-to-layer pressure.

The use of the friction tabs was followed with an analysis program which compared theoretical and experimental results. A method for estimating the periods between tape rewinds was given high priority. It was determined that if the tape is wound at a higher temperature than the storage temperature, or wound at a lower humidity than the storage humidity then the allowable period between rewinds is increased. This is due to the reduction in both the reel tensions and the tape creep.

\subsubsection{Preparation Phase}

(1) Quality of Tapes that are Chosen

(a) State-of-the-art (i.e., late production types) of computer tapes appear to be potentially successful candidates for archival storage purposes.

(b) Optimally, the tapes should be new and should have been maintained in a cool and dry environment prior to purchase.

(c) If possible, it should be ascertained that the archival candidate tapes have not been subjected to any environment which could have caused polyurethane binder deterioration due to binder hydrolysis.

(d) The magnetic particle material used in the computer tape should be gamma ferric oxide $\left(\gamma-\mathrm{Fe}_{2} \mathrm{O}_{3}\right)$, at present, since its ability to maintain its magnetization for long periods of time has been established. No other presently available particles have yet proven to be of equal long-term stability at this time for similar temperature-humidity ranges.

(e) Use backcoated tapes. Backcoated tapes provide increased interlayer friction which reduces the probability of layer-to-layer slippage due to tape relaxation during storage. An additional benefit for backcoated tapes has been noted during SRM calibration procedures by the ICST Computer Storage Media Group: the signal amplitude levels reproduced from these tapes are generally more uniform, with much smaller end-to-end signal level rise effects than are usually observed with non-backcoated tapes. 
(f) It is not feasible for tape acceptability criteria to include the individual tape binder constituents since they are almost always considered to be company proprietary. It is suggested that the most reasonable constituent type qualification tests are achieved indirectly through the measurement of the hydrolysis tendencies [28],[29],[30] of the binder systems and the stability of the magnetic particles. (Note: It is reasonable to assume that these media will also be subjected to the overall requirements of the GSA computer tape specification $\mathrm{W}-\mathrm{T}-0051 \mathrm{C}$ which is described in sec. 2.1.1.)

(g) Candidate archival tapes should be unsealed and allowed to relax for three days in the storage environment of $17^{\circ} \mathrm{C}\left(62^{\circ} \mathrm{F}\right)$ to $20^{\circ} \mathrm{C}\left(68^{\circ} \mathrm{F}\right), 35 \%$ to $45 \% \mathrm{RH}$ for several days after receipt. This conditioning is particularly necessary if the intermediate transit environments are not known. It also allows the tape to come into hygroscopic equilibrium, in the storage environment. After relaxation, the tapes should be given two complete, slow speed passes and then evaluated for dropout errors. It is suggested that the large population error criteria in section $2.1 .1-(3)$ be considered. If there is a large error rate, a cleaning pass is suggested, followed by tape evaluation.

(h) The following test is recommended as a means for testing tape binder integrity: The tape is stored for 72 hours in an environment of $49^{\circ} \mathrm{C}+3^{\circ} \mathrm{C}$ $\left(120^{\circ} \mathrm{F}+5 \mathrm{OF}\right), 80 \%$ to $90 \% \mathrm{RH}$, and then stabilized for 16 hours at $24^{\circ} \mathrm{C}$ $\left(75^{\circ} \mathrm{F}\right), \overline{5} 0 \% \mathrm{RH}$. The test results as applied to a computer tape are as follows: there should be less than a doubling of the original dropout rate and very little coating shedding in an acceptable tape which is scheduled for archival use after being subjected to the preceding environmental conditions. It is noted that an archival tape candidate, as a minimum, should pass this 72 hour test.

(2) Physical Preparation Prior to Long-Term Storage

(a) Store the archival tapes in a dust-free environment in order to reduce dropout accumulation.

(b) Tape reels should have aluminum flanges for long-term support.

(c) Place in well-sealed polyethylene bags in order to prevent long-term binder lubricant evaporation, and perhaps reduce binder degradation tendencies.

(d) Tapes should be boxed and stored in a vertical standing position.

(e) Tape reels that are not in canisters should be secured with wrap-arounds at all times, both in and out of storage in order to protect against tape edge damage due to flange compression or distortion.

(f) Always store tapes, with their windings oriented in the standard ready-to-read or write position after a slow speed rewind. This applies both before and after recording the archival computer tapes. Constant tension winding appears to be satisfactory for computer tapes. 
(g) A slightly higher winding area temperature compared to the storage area temperature, or a slightly lower winding area humidity compared to the storage area humidity will prolong the allowable period between tape rewinds. It is suggested however that only relatively small area differences, i.e., $2.8^{\circ} \mathrm{C}\left(5^{\circ} \mathrm{F}\right)$ and $10 \% \mathrm{RH}$ should be allowed in order that tape relaxation times will be minimized when passing from the storage area to the computer area.

\subsubsection{Storage Phase}

(1) Static Recommendations

(a) The tapes should be stored in archives within the $\mathrm{T}-\mathrm{H}$ range of:

Temperature: $17^{\circ} \mathrm{C}\left(62^{\circ} \mathrm{F}\right)$ to $20^{\circ} \mathrm{C}\left(68^{\circ} \mathrm{F}\right)$

Relative Humidity: $35 \%$ to $45 \%$

and optimally at:

Temperature $=18.3^{\circ} \mathrm{C}\left(65^{\circ} \mathrm{F}\right)$

Relative Humidity $=40 \%$

Note: The optimal temperature and humidity are considered to be a key finding of this research program [22].

(2) Dynamic Recommendations

(a) Imbed friction tabs on a semimonthly basis into a chosen group of unrecorded test tapes of the same type and size as the actual recorded archival tapes. These tapes must be wound with the same tensions and tension-patterns as the recorded stored tapes. Store these tapes in archives and perform a tab pull test also on a semimonthly basis in order to determine when the critical layer-to-layer pressure has been reached and rewinding is necessary. (Note: Although [22] does not give quantitative information relative to critical tab pull forces for computer tapes, it does offer background information which can be used to set up individual test programs by interested organizations.)

(b) Rewind each tape in archives after it has been stored for 3.5 years, providing that:

1. The storage area temperature and humidity has remained at its optimal value given in section 2.2.3-(1) for the entire period, and,

2. The friction tabs pull forces have not yet reached their critical values. Rewind each tape immediately after the critical value has been reached, regardless of its time in storage.

\subsubsection{Reactivation Phase Recommendations}

(1) Give tapes two full forward and reverse passes at nominal (slow) speeds before attempting the read process. 
(2) It is advantageous to perform this read process in a controlled environment as close to the archival environment as possible; otherwise permit the unsealed tapes to relax in the new environment for at least three days before at tempting the read process.

(3) If environmental damage to the tape is suspected (i.e., such as an increase in binder hydrolysis), store the tapes unsealed for three days in the archival storage area at the recommended temperatures and humidities (see sec. 2.2.3). Then reread the tapes and clean if the error rates are high.

(4) When the reading is completed, reseal the tapes and return to storage, if required.

Author's Note: It is suggested that the recommendations given for the large population cases in section 2.1 ; i.e., for the material preparation phase, the static and dynamic storage phases, and the reactivation phase, be selectively applied in conjunction with these small sample (sec. 2.2) recommendations. This refers to those large population recommendations which are not in conflict with any of these preceding small sample recommendations. 


\section{Scheduled Maintenance of Computer Magnetic Tapes}

The scheduled maintenance of computer magnetic tape libraries and vaults consists of principal activities such as tape cleaning, tape evaluation (or certification), and tape rehabilitation (see secs. 1.13 and 1.14). It also includes associated activities such as tape exercising and cutting (stripping). The cleaning process removes loose error producing debris from the tape before it becomes permanently fused into the tape surface; the evaluation and certification process detects, counts, and locates the errors on the tape surface; the rehabilitation process incorporates several of the preceding activities for the purpose of restoring computer tapes to a useful status.

\subsection{Computer Tape Errors}

Many serious error problems which are encountered during computer tape use are caused by the self-contamination of the media. That is, the media itself produces a large amount of the debris wear products which become redeposited and permanently fused into the tape coating (see sec. 1.3-(12)); these eventually cause dropout errors and data losses. The errors result from the subsequent read/write head-to-tape separation caused by the surface irregularities which have been produced by the lumps of embedded tape debris. It has been found that once these wear products have started to collect on the surface of the tape, the rate of production of additional wear products increases rapidly and the performance of the tape also deteriorates rapidly. In fact, the deterioration of the tape performance is usually more a function of the redeposition of these wear products than the condition of the tape surface as related to tape usage or coating thickness. Note that each system retry which is necessitated by a tape write-skip error is in itself a debris producing activity; therefore, the more write-skip errors there are, the faster is their rate of increase when retry methods over an area are applied. The principal purpose of the tape cleaning process is to remove the loose debris particles from the surface of the tape before they become fused into the surface and reach excessive or critical quantities. However, it should be noted that although the useful life of a computer tape is extended by a regular tape cleaning program, there still remains a slower but steady growth in permanent errors during the cleaning program. That is, the fused debris error count continues to increase towards an irreducible value for which it is no longer cost effective to clean or to continue to use this tape (see sec. 3.1.1(c)).

Permanent errors on magnetic computer tapes may be defined either as a physical or a sof tware type as follows:

Type (a) - Physical: errors which are caused by surface defects such as fused, irremovable particles in the tape surface. Tapes containing these permanently fused particles are usually acceptable for continued use provided that the particles don't exceed a specified quantity which is established by the individual organization. However, these particles can cause tape rejection if they are located as described in (b) below. The following two permanent error types usually result in a tape reject during operation or rehabilitation:

Type (b) - Software: permanent errors which consist of a specific number of consecutive write skip errors or retries. For example, several major sof tware (operating) systems define such a permanent write error as one in which 15 consecutive write skip errors occur approximately $0.1 \mathrm{~m}$ (4 in) apart over a 
$1.5 \mathrm{~m}$ (5 ft) length of computer tape. Note however that in these systems it is possible to have a non-rejected tape with 15 write skip errors (caused by type (a) defects) provided that they are not located consecutively as defined in this paragraph.

Type (c) - Physical: gross errors which are the result of permanent physical tape damage such as creases, folded edges, or missing coating material which extend over a length of the tape. Typically, these regions are detected as gross errors when they are approximately $6.3 \mathrm{~mm}(0.25 \mathrm{in})$ long (see table 4.$)$.

Temporary errors are usually defined as errors which are caused by loose particles whose position can be shifted by the normal sweeping action of the tape against the head or the guides. However, if these particles are not removed through cleaning, they multiply rapidly and eventually become permanently fused into the surface. If properly placed, these temporary errors can eventually lead to the reject defined in type (b) above.

The basic formula which determines the head-to-tape separation signal loss caused by the debris particles or other surface protrusions (see sec. 8.1-eq(4)) indicates that for the same size debris, the signal loss (in $\mathrm{dB}$ ) will be much greater for $246 \mathrm{bpmm}$ (6250 bpi) recording than for $32 \mathrm{bpmm}(800 \mathrm{bpi}$ ). Therefore, tapes which are used for higher densities are more susceptible to signal loss (drop-outs) and must be kept free of debris.

\subsubsection{Scheduled Maintenance Cost Factors}

(a) The Scheduled Computer Tape Maintenance Recommendations in section 1.14 outline a number of optional factors which can be used for deciding when specific maintenance activities should be initiated. These include:

- The number of times the tapes have been mounted to date.

- The number of times the tapes have been cleaned or evaluated to date.

- The operating condition of the tape; i.e., the number of tape errors.

- The expiration date of the tape data.

- The length of time the tape has been in storage.

- Regular maintenance of new tapes before use.

The decision parameters which underlie a particular scheduled tape maintenance program are also based upon both an operational and a cost/benefit analysis of the organizations needs. For example, the number of allowable errors before tape maintenance is performed, must be weighted differently by an organization in which data errors affect specific output quantities, say, the amount of a Social Security check; while data errors which enter into a statistical data base such as a Census program are individually of much less significance in their impact on the final results. 
The frequency of required tape maintenance is also influenced by both the age and history of the tapes, and occasionally by the manufacturing source of the media. If the data relative to the tape errors result in statistical distributions which indicate problems with particular types or brands of tape, or modes of operation, then scheduling adjustments and other actions can be instituted.

Extensive record keeping of the chosen factors may be required in order to produce the scheduled flow of tapes into the maintenance process. This record keeping can be performed by Tape Management Systems (see secs. 4.1 to 4.3), by error-logging software systems which are capable of testing tape surfaces on-line (for example, see sec. 4.4), or by manual check-off systems (in small user organizations - see sec. $1.2-(19))$.

(b) The cost/benefit analysis includes factors such as:

- Maintenance equipment costs.

- Maintenance personnel costs.

- Contractor costs if performed by an outside organization.

- The size of both the active library and the tape archives.

- The number of inactive tapes which could be rehabilitated back into active use; i.e., potential savings on the costs of new tapes.

- The total maintenance costs per tape compared to new tape replacement costs; i.e., at which point is it cost effective to purchase a new computer tape rather than to continue with the cleaning, evaluation, and rehabilitation processes. This maintenance versus replacement breakpoint depends upon factors such as the average number of write errors per active tape and their annual cost in CPU time for the particular operating system.

(c) Whereas permanent errors type (b) and (c) in section 3.1 normally lead to a job abort and the rejection of a computer tape, type (a) write skip errors constitute a key cost item for non-rejected tapes which remain in a continuing operating mode. These operational costs are a function of the costs of the write-skip retry processes which are software dependent.

Since the number of type (a) write skip errors increases rapidly with tape use, eventually the number of these errors will reach a value at which it is no longer economically feasible to use a tape in this condition. An estimate of both the yearly losses due to write skip errors and when a tape should be replaced, due to write-skip error accumulation, can be obtained for a particular installation as follows:

(1) Compute the average number of write skip errors per day based upon the computer printouts from the error-logging system.

(2) Compute the cost of a single type (a) write skip error. In making this computation, base the single write skip error cost on the time which is lost while automatic, write retries are performed in the write error area. This 
is dependent upon both the operational costs per unit time of the CPU as well as the system software; i.e., the number of retries made before skipping ahead or the number of retries before the final decisions made, is sof tware dependent.

(3) Multiply (1) by (2). This gives the average costs for write skip errors each day.

(4) Multiply (3) by the number of operating days per annum. This gives the average total money loss yearly due to write skip errors alone. This may be a very revealing amount which can be useful for making decisions relative to the initiation of a scheduled tape maintenance program.

(5) Determine what percentage of the tapes in the total active library are mounted each day. From this, calculate the average number of times a tape will probably be mounted per annum by multiplying the above percentage by the number of operating days. Equal probability for each tape is assumed.

(6) Multiply (5) by (2). This gives the average write skip error cost per annum for each active tape assuming only a single write skip error on each individual tape each time it is mounted. Replace a tape when the calculated number of its accumulated write errors times the average yearly single error cost is equal to the cost of a new tape. This is reasonable, since with further usage, the operating costs for the tape will now begin to exceed the new tape costs.

\subsection{Types of Computer Magnetic Tape Maintenance Equipment}

The characteristics of some of the available tape maintenance equipment such as cleaners, certifiers, and evaluators will now be discussed. The following descriptions are not intended to be all inclusive since there are many models of each instrument, and many other technical features available. Information relative to specific models can be obtained from the equipment manufacturers (see [10] and [11]).

\subsubsection{Tape Cleaners}

A tape cleaner typically has a reel-to-reel configuration which is similar to a tape transport. Its basic functions are to clean and then properly wind and tension the computer magnetic tape. Recorded tapes are normally not erased during the cleaning process.

Typical cleaners for $12.7 \mathrm{~mm}$ ( 0.5 in) magnetic computer tapes will accommodate tape reels which are up to $267 \mathrm{~mm}$ (10.5 in) in diameter. These machines transport the tape over both tissue wiping stations as well as sharp cleaning blades. Some units pass both sides of the tape over the tissues on the forward and reverse passes; this removes the debris from the back (mylar) side of the tape before the particles are transferred to the coating side. Other units remove the debris with vacuum devices which are incorporated into their cleaning blades. These blades are made of various configurations and materials which include: gemstones, such as sapphire; self-sharpening, stainless steel, perforated metal grids; rotating, self-sharpening, 
slotted, stainless steel cylinders; rotating flexible stainless steel bands which continuously contact their sharp edge against the tape surface; and simple stainless steel blade types.

Each type of blade mechanism has advantages and disadvantages. For example, the simple stainless steel blade displays superior cleaning performance but has an extremely short life, while a slotted steel cylinder has a very long life but does not clean as effectively as a blade. In addition, the simple blade when worn is more likely to cause tape damage than a slotted cylinder blade. One unit has an automatic blade life indicator which prevents tape edge damage that can be caused by irregularly worn blades.

Most cleaners maintain uniform tape winding tensions in both the forward and reverse directions in order to produce a good tape pack. In addition, some tape cleaners apply a greater than normal tape tension during the cleaning pass, and a normal tape tension during the rewind cycle; this produces a more effective cleaning cycle. The tape winding speeds vary among different cleaning units. Some units are relatively slow with speeds of $2.3 \mathrm{~m} / \mathrm{s}(90 \mathrm{in} / \mathrm{s})$ to $2.5 \mathrm{~m} / \mathrm{s}(100 \mathrm{in} / \mathrm{s})$ while others are capable of speeds up to $9.1 \mathrm{~m} / \mathrm{s}(360 \mathrm{in} / \mathrm{s})$ for faster production in large tape lots.

Some tape cleaners which are capable of erasing data have an automatic file protect feature which prevents the accidental erasure of recorded tape information. Most cleaning units are capable of BOT and EOT sensing as well as automatic forward and reverse features.

\subsubsection{Tape Certifiers}

A tape certifier typically has a reel-to-reel configuration which is similar to a tape transport. Tape certification is performed in order to detect, count, and locate tape errors, and then, if possible, repair the underlying defects so that the tape can be replaced back into an active status. In addition to performing these specialized operations, certifiers have the same basic tape cleaning capabilities as a typical tape cleaner. Cleaning is normally performed prior to testing in order to reduce or eliminate temporary error counts. Sometimes the cleaning is performed on the forward tape pass, and the certification measurement is then performed on the reverse pass. Typically, certifiers have 7 or 9 track read/write heads and will operate at various selectable recording densities (see "Certifiers, Magnetic Tape [10]). Note that the error rate counts will usually increase as a function of increasing recording density (see sec. 8.1-eq(4)).

During certification, previously recorded information is erased from the tape and new pulse trains are written on each track; a read-while-write process is then performed on each track of the tape under test. Although certification is normally a data destructive process, it is possible to perform non-destructive testing with some error-logging systems (for example, see sec. 4.4). Also, if a Clean Only mode of operation is desired, then the write inhibit ring must be removed from the tape reel; it must be inserted for the TEST mode. The certifier is usually calibrated with a test tape so that the amplitude of the test signal is related to a specific preset reference level. This level is usually traceable to one of the National Bureau of Standards magnetic media Standard Reference Materials such as SRM 3200 or SRM 6250. In some cases, the reference level is related to an internal, organizational tape signal level. 
Upon readback, if the test signal amplitude falls a specific percentage below the preset reference level, the certifier will retry the read/write processes over the same location. Some certifiers allow for an adjustable number of retries, say, 0 to 12 times, before stopping for an examination at the defective area if the error persists for the chosen number of retries. The track(s) on which the error occurs is also detected and recorded. When the certifier stops at the defect, the operator of the unit can locate and examine the defect region microscopically. If possible, the operator will attempt to remove the defect either with a chemical such as Freon TF or by physically scraping the defect with a scalpel and then removing the residue with Freon TF. This chemical is an excellent tape cleaner since it does not soften the tape oxide or the backing, or damage rubber capstan idlers [21]. Certifiers can also test for defects known as tape noise errors, skew errors, and level errors which are caused by excessively low or high output signals.

Typically, if removable errors are encountered and repaired, the tapes can be restored to full service. If only a few type (a) permanent errors (see sec. 3.1) are encountered, it is possible to return the tape to lower level scratch service, while if there are many type (a) permanent errors, the tape should be discarded.

\subsubsection{Tape Evaluators/Testers}

These instruments will perform most of the same cleaning/testing functions as tape certifiers with the exception that they normally do not stop the tape when an error is encountered unless an automatic stop feature is added as an extra instrument function. Whereas a certifier is well fitted to activities in the product test area of a media production plant where tape defect removal may be attempted, an evaluator is well fitted to a test area such as a tape rehabilitation center (see sec. 3.3) in which tapes are tested on a go-no-go basis with no at tempt at error removal.

The following is an example of the functions in one tape evaluator system: This evaluator performs its cleaning and testing functions both in the forward winding direction. The number of permitted errors depends upon organizational decisions; for example, say only five consecutive write skip errors are permitted before the tape is rejected. This evaluator contains an internal microprocessor which oversees the tape evaluation, and the reporting and printing of the results. It records and counts the total number of dropout errors, and provides a digital printout which gives the location of these errors (in feet) from the Beginning of Tape (BOT) marker. It will also measure the length of tape under test from the BOT to the EOT (End of Tape) markers. This system winds the tape with a constant tension and can record and perform measurements on either 7 or 9 track read/write heads, at $32 \mathrm{bpmm}$ (800 bpi), 63 bpmm (1600 bpi), or 246 bpmm (6250 bpi).

The functional sof tware characteristics of evaluators typically provide performance which is designed to be compatible with the sof tware for the computer tape drives in use. For example, some widely used operating systems (such as OS and DOS) have I/O sof tware which does not call for tape backspacing in order to retry a tape write error. Instead, the tape is moved forward $102 \mathrm{~mm}$ (4.0 in) after a write error is detected; then the data is rewritten and reread again. Now if 15 consecutive write errors (skips) occur, the system will consider a permanent error to have occurred and the job is aborted and the tape is rejected. It is therefore realistic and advantageous to employ evaluators which perform their error search functions under 
the same conditions as the organization's operating systems. There are various evaluators available which do their error search in different ways including the preceding non-backspacing mode. An example of the multiple error criteria which can be tested for by a single evaluator is incorporated in table 4 .

\subsubsection{Miscellaneous Devices}

In addition to the cleaners, certifiers, and evaluators, there are several other instruments for use with computer magnetic tapes. One is the bulk ac tape eraser or degausser; the other is the programmed tension winder. The bulk ac eraser will remove all previous magnetic recording from the computer tape and leave the media in a fully demagnetized state with no magnetic bias remaining in either polarity. This process is a form of anhysteric demagnetization. The programmed tension winder produces a tension pattern throughout the tape pack that eliminates the zero tension tape layers which lose their layer-to-layer friction and cause tape cinching damage upon reel acceleration or deceleration by the transport. Programmed tension winding is particularly useful prior to long-term or archival storage (see sec. 7.4).

\subsection{Tape Rehabilitation}

As previously mentioned, the objective of tape rehabilitation is to restore used computer magnetic tapes to a fully useful status. There are a number of organizations which perform the complete tape rehabilitation process for smaller companies that cannot justify the costs of the required equipinent, or for larger organizations for whom rehabilitation becomes a major undertaking. Typically, the company which submits the tapes for rehabilitation will first recopy the data, which it wishes to retain, onto new tapes prior to shipment for full rehabilitation. Some equipment vendors have rental or leasing plans for their tape cleaners, certifiers, and evaluators. There are also commercial contractors who will set up and operate a rehabilitation facility directly at the user site for organizations with large tape libraries. This permits the tapes to remain in their normal temperature-humidity environment and eliminates potential shipping damages (see sec. 1.5). It is suggested that these contracting organizations can be of assistance in making initial decisions relative to the cost/benefits and technical problems involved in setting up a tape rehabilitation program.

An example of a rehabilitation process which is performed by a contractor on-site for a large Federal agency tape user (Social Security Administration) has some of the following features:

- Five thousand tapes are rehabilitated every 24 hours.

- Every 24 hours when data tapes are released for rehabilitation they are either "cleaned only" or "cleaned and evaluated."

- All new tapes are cleaned and evaluated before first use.

- Upon completion of the rehabilitation processes, the contractor must perform quality assurance testing and the agency will perform acceptance testing of completed lots. 
The following are some of the mandatory contract obligations, definitions, and activities which are observed and performed by the contractor:

\subsubsection{Class of Work}

(a) Clean only: During a clean only pass, the tape must be cleaned and then wound at the proper tension onto a clean reel. Proper tension is a tension which is compatible with those of the Federal agency's tape transports. The original external labels must remain on the clean reel or be recopied for placement onto a new reel if the original reel is not used for the cleaned tape. Note that a "clean only" pass is always performed when the data is to be saved.

(b) Clean and evaluate: The tape must be given two complete passes; one through a cleaning cycle and one through an evaluation cycle. The tape is then wound onto a clean reel.

\subsubsection{Product Requirements}

The following contract requirements describe the final product of the facility, i.e., a rehabilitated tape.

(a) Container: The container, be it canister, self-threading cartridge, or some other device which protects the reel of tape, must be clean and free of dents, cracks, or defects which might inhibit continued use of the container for its intended purpose.

(b) Reel

(1) The reel must be free of nicks, cracks, warping, or other defects which might inhibit continued use of the reel for its intended purpose.

(2) The reel must be clean. It must contain no other labels except the following:

a. The manuf acturer's label

b. The agency's volume serial number

c. The contractor's process number label

d. Green, yellow, and red dots (for track and density identification)

(3) The reel must have a properly installed write enable ring.

(c) Tape

(1) The tape must be in a range of the following track formats and densities: 9 -track, $63 \mathrm{bpmm}$ (1600 bpi) and $246 \mathrm{bpmm}$ (6250 bpi). The color of the dot, or lack of any dot, indicates track and density char acteristics.

(2) The tape must be clean of all unbonded and substantially all self-bonded and environmental dirt, oxide, backing fibers, and other foreign material on the tape surface, front, back, and edges. 
(3) The tape must be free of all physical defects, including but not limited to abrasion, tears, grooves, splicing, stretching, creases, and edge damage.

(4) Tapes which are rejected at $246 \mathrm{bpmm}$ ( $6250 \mathrm{bpi}$ ) may not be retested $63 \mathrm{bpmm}$ (1600 bpi), unless directed to do so by the contracting officer's representative. The contractor shall provide a permanent record of both the number and approximate location of errors for each tape.

(5) Both temporary and permanent errors will be counted. Table 4. shows the maximum dropouts and/or errors allowed per reel in the rehabilitation facility and in Quality Assurance. Note that the contractor's maximum error counts are less in order to provide a safety margin to help prevent tape lot rejections.

(6) The tape must have correctly positioned and acceptable BOT (Beginning Of Tape) and EOT (End Of Tape) markers.

(7) The tape must be tested from the BOT reflective marker to a minimum of $0.61 \mathrm{~m}(2 \mathrm{ft})$ past the EOT reflective marker.

(8) A capability that will allow a tape to be cleaned and evaluated while maintaining the integrity of the internal labeling conventions is a mandatory feature.

(9) Minimum acceptable tape length (BOT to EOT) is $671 \mathrm{~m}(2200 \mathrm{ft}$ ).

(10) The tape must be wound at a winding tension compatible with the agency's tape drives $(1.7 \mathrm{~N}(6 \mathrm{ozf})$ to $2.8 \mathrm{~N}(10 \mathrm{ozf}))$. The tape pack shall form a smooth, integral mass that is not loose either axially or circumferentially, and the finished tape pack shall be centered on the reel hub. Tape must be wound, oxide surface toward reel hub, in a clockwise direction, when the reel is viewed from the front, the loose end of the tape hangs from the right side of the reel.

(11) The standard testing environment must be a normal computer room environment.

\subsubsection{Record Keeping}

(a) Each reel must be marked and a peelable label must be used on each reel in order to identify the following:

(1) Date evaluated

(2) Operator who performed the evaluation

(3) Evaluator used (Machine)

(4) Tape length in feet

(5) Number of errors 
TABLE 4. MAXIMUM DROPOUTS/ERRORS PER REEL

$246 \mathrm{bpm} \mathrm{m}(6250 \mathrm{bpi})$

No more than 50 single-track errors 26

No more than 10 two-track errors

No more than 3 three-track errors

No gross errors 27

No permanent write errors 28

No more than 7 marginal edge errors

No more than 4 marginal edge errors

in any $0.90 \mathrm{~m}(3 \mathrm{ft})$ section

No more than 25 errors combined which

are comprised of single-track,

two-track, three-track, and edge errors
No more than 55 single track errors

No more than 12 two-track errors

No more than 4 three-track errors

No gross errors

No permanent write errors

No more than 8 marginal edge errors

No more than 4 marginal edge errors in any $0.90 \mathrm{~m}(3 \mathrm{ft})$ section

No more than 30 errors combined which are comprised of single-track, two-track, three-track, and edge errors

$63 \mathrm{bpmm}(1600 \mathrm{bpi})$

\section{CONTRACTOR}

ACCEPTANCE TESTING
No gross errors

No permanent write errors

No more than 7 marginal edge errors

No more than 4 marginal edge errors in any $0.90 \mathrm{~m}(3 \mathrm{ft})$ section

No more than 10 dropouts

No more than 12 errors, edge, and dropouts combined
No gross errors

No permanent write errors

No more than 8 marginal edge errors

No more than 4 marginal edge errors

in any $0.90 \mathrm{~m}(3 \mathrm{ft})$ section

No more than 12 dropouts

No more than 14 errors, edge, and

dropouts combined

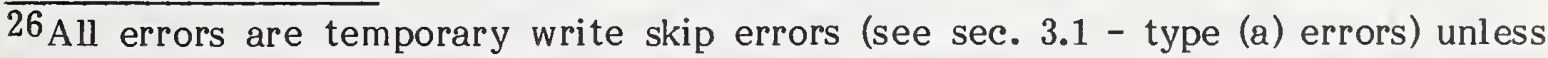
noted as permanent or gross.

$27 \mathrm{~A}$ gross error is usually caused by scraped oxide, diagonal creases, etc., and typically persist for at least $6.35 \mathrm{~mm}$ (0.25 in).

${ }^{28} \mathrm{~A}$ permanent write error is a software dependent error and is defined, for example, by the number of consecutive write skips over a length of tape (see sec. 3.1 - type (b) errors). 
(b) Unless otherwise specified, the contractor must maintain, for the term of the contract, original test records, data sheets, etc., which will be available for the tracing of each rehabilitated tape. The individual records for each tape shall include lot number, the agency volume serial number, machine, operator, number of errors per tape, date evaluated, length of tapes, and which components had to be replaced. The records are available for inspection by the agency on demand.

(c) The contractor prepares and submits a detailed monthly summary of the rehabilitation process performed. These records shall include, but not be limited to, a production summary to date, the percentage of tapes by recording format, a summary of tapes received, total accepted and rejected quantities, and percentage of yield.

\subsubsection{Quality Assurance Requirements}

(a) The contractor must establish and maintain a quality assurance area so that rejected lots will be kept at a minimum.

(b) Acceptance testing of completed lots will be done by the agency's quality assurance personnel on a statistical sampling basis. The samples will include both tapes certified as acceptable and those certified as unacceptable by the contractor.

(c) Each finished unit in the sample shall be given a thorough visual and dimensional inspection to determine compliance with the requirement of the agency's specifications. Particular attention shall be given to, but not limited to, the following:

(1) Cleanliness

(2) Tape dimensions (length)

(3) Tape wound pile

(4) Reel dimensions

(5) Marker placement

(6) Error count

(d) Lots rejected by the agency's quality assurance personnel will be returned to the contractor, with identification as to what will be required to correct the lot. The contractor will not be reimbursed for the correction of rejected lots.

At the present time the agency's objective is to produce a $75 \%$ successful yield from all rehabilitated tapes.

No effort has been made in this particular rehabilitation process to excise (strip) defective tape regions (particularly at the beginning of the tape) or to microscopically remove defects as described in the section dealing with tape certifiers. In addition, unlike some other rehabilitation processes, there is no environmental tape relaxation period or specialized final tape winding, such as a programmed tension wind. 


\section{Tape Management Systems (TMS) 29}

Methods for the proper care and handling of computer magnetic tapes are available for application at all levels of activity beginning at the system software level and continuing through the hands-on activities of the personnel in computer installations, tape libraries, archives, testing laboratories, and external environments. In their scheduled passage through these areas, it is possible that the media and/or their data contents may be lost or destroyed due to the lack of proper record keeping or traffic control methods.

One approach to these logistical problems, particularly in large installations, lies in the application of sof tware systems which are known as Tape Management Systems (TMS). These TMS interact with the data processing system and provide total organization and control of the flow of the computer tapes in, through, and out of the data processing system, including libraries and vaults. In addition, they can label, protect, retain, withdraw, scratch, 30 quality control, identify, schedule, pre-stage, control flow both on and off site, and locate the position of every computer tape in the system.

This section discusses those specialized areas found in three Tape Management Systems whose functions are to earmark, control, and trace the movement of the media and their data through the various operations. They maintain the security of the data by circumventing system operations which can pose a threat to the media and data. It should be noted that in these and most other TMS there are a number of other important functional capabilities which are related to areas such as data organization and cost effective media operations. For example, one source claims that a TMS can liberate enough system capacity so as to permit up to $30 \%$ more tape processing throughput [32]. Some of these TMS are quite complex and costly.

In addition to these complete Tape Management Systems, there are limited error-logging software systems which deal solely and directly with the condition of the computer tapes. One of these systems, SAS-I (see sec. 4.4), can be used in lieu of off-line tape evaluation and certification methods which were described in the Scheduled Tape Maintenance section 3.2.3.

The brief discussion of the following three TMS and the SAS-I to the exclusion of other available systems is not to be construed as an endorsement of any particular systems. They were chosen as examples because of their direct relevance and applicability to the tape care and handling process. Interested readers can obtain more complete information from the vendors of available systems.

\subsection{TMS-I}

This system [33] automates the record keeping of a tape library and vault and produces reports for their daily operation and control. It provides the user with a full facility for inquiring into and maintaining complete information pertinent to the operation of the tape library. It accommodates a library which is organized by tape volume, serial number, or by application.

${ }^{29}$ These systems are also referred to as Tape Library Management Systems. $30 \mathrm{~A}$ scratch tape is one which contains no useful data and is capable of being reassigned to a new application. 
TMS-I provides both a daily tape movement report and a tape inventory by location report. It also offers a real-time verification of the scratch eligibility of an output tape volume at open time. Gummed labels with complete up to the minute information can be produced on-line during the actual execution of the job at the time the tape is created. These labels can be generated in a variety of ways which are selected by the user; this includes both on-line or batch mode operation. If desired, the user may not call for any labels.

TMS-I has several fail-safe backup capabilities such as the automatic restart at the point of the tape activity which is in process at the time of a system crash. The system can produce a variety of standard reports at the user's discretion. Among these are a complete list of tapes under retention, eligible scratch tapes, scratch forecasting, tape movement, offsite storage inventory, and a compiete tape inventory. These reports can be accessed by referring to such items as the tape volume serial number, data set name, and the creation date.

TMS-I can also report the following type of computer tape information upon request: purchase date, manufacturer, recording density, type of label, footage, total times used, location, date last cleaned, times used since last cleaning, read errors, write errors, and the responsible programmer.

TMS-I is capable of tracing a data set through previous generations even though its name has been changed. It will process data differently depending upon whether the on-line or the batch mode of operation is applied.

TMS-I automatically screens the quality of all tapes as they are moved into the scratch pool. This includes tape age, tape cleaning information, and tape error statistics. Flags indicating quality variances are displayed on a Daily Scratch Tape Report. In addition, when tapes pass their retention date, they will also appear on this report.

Every major function which is provided on-line is completely duplicated in the batch backup system. This means that if for any reasons the on-line system fails to function, TMS-I provides the user with an automated batch backup system. These backups include such major functions such as tape label generation, update for newly created tapes, and newly available scratches among others.

\subsection{TMS-II}

TMS-II [34] provides protection against the inadvertent destruction of tape files and permits tape operation with minimum operator intervention.

The central control in TMS-II is provided by a Tape Management Catalog (TMC) which prohibits any processing of a tape by a user program unless the TMC first validates the operation. All necessary information associated with each tape volume is stored immediately in the TMC at tape OPEN, CLOSE, or EOV operation.

The TMS-II management system handles standard labeled, non-standard labeled, and unlabeled tapes. The system removes the need for creating and placing physical labels containing data set information on the tape reels; only the volume and serial numbers are required. This eliminates the possibility of an operator assigning a wrong label to a reel of tape. It is possible to operate under TMS-II control without 
removing the tape write enable rings. Each secondary backup file on a tape is separately protected and reported with its own identifying record and expiration date in the TMS-II data base. There is a data set (AUDIT) which tracks all changes to the TMS-I data base. This provides for the restoration of the data base up to the instant of failure should one occur.

TMS-II is capable of reporting the following type of information (and others not listed):

(1) Tape inventory lists by volume serial number, by data set, and job name.

(2) Forecasts of tape to become scratch.

(3) List of scratch tapes with cleaning information.

(4) A list of inactive tape reel numbers.

The system will also produce a list of all tapes which have generated I/O errors in the past 48 hours. It can provide data set security through its own PASSWORD facility which is independent of the operating system. This password facility prevents access to sensitive data as long as the tape is under TMS-II control. This security however cannot be assured if the tape is sent to an uncontrolled area or is scratched. To prevent this, a utility program is available in TMS-II for erasing sensitive data before the volume is sent out.

TMS-II provides the following type of retention criteria for tape data sets: expiration date, retention period, frequency control (i.e., a tape data set is held as long as it is used every $x$ number of days), permanent tape hold, etc. There is a program which lists all tapes with a retention period of one year or longer.

It also provides lists of scratch tapes which should be cleaned, based upon criteria such as the number of days since the last cleaning, number of uses, number of temporary read or write errors, or any combination of these which the user has specified (see sec. 1.14). Tape certification processes can also be tracked by the system.

There is a special area in each tape volume record in the Tape Management Catalog (TMC) which is available for storage of infor mation, such as the tape manufacturer's name, certification information, or any other useful information relative to effective tape management.

TMS-II incorporates a Vault Management System (VMS) which controls the movement of tape volumes from one storage location to another. Typically, critical tape volumes are cycled out of the central tape library to progressively more secure and less accessible storage areas such as vaults, and then are finally transferred back to the central library. The VMS maintains a record of all vaulted tapes. For example, when a tape meets the criteria for being vaulted, they are listed as being "out-of-area" with the proper vault code and records of the current location and destination for tapes that are to be transferred. The vault tapes can be located by slot numbers which are automatically controlled and updated by VMS. The retention of a vault tape can be based on one or more criteria such as number of days elapsed since placed in vault, number of days since the tape was created, a specific hold time, plus several others. 
One large user of computer tapes in the Federal Government employs TMS-II system in order to keep track of 7,000 to 8,000 reels which they mount daily. The system generates reports such as: summaries of temporary and permanent errors encountered on each mounted reel, identification of tapes in storage for ten years, five years, etc., the manufacturer and brand name of each tape. Critical tapes are backed up daily with multiple copies; one set is stored off-site.

\subsection{TMS-III}

This system maintains complete control over a tape library. This control extends into the areas of data security and data set inventory management [35].

The TVIS-III system maintains a master file which has a real-time record of every tape volume. The master file data are obtained from the operating system whenever tape data sets are read or written. The master file is accessible for both on-line inquiry and off-line generation of reports. The data sets are protected by the TMS-III Data Set Protection feature against unwanted destruction by the operator unless their release is authorized. It recognizes all protected tapes and prevents them from being written over by automatically dismounting them.

The master file maintains information such as the purchase date of the media, the manufacturer, the last date when the media were cleaned and certified, the location of the tape, the number of permanent write and read errors. The system will highlight tape volumes that have a high failure potential due to excess errors and will also automatically schedule preventive tape maintenance (see sec. 1.14). TMS-III exerts automatic tape scratching control; this decreases the total number of tapes which remain in the operation. Also, through various other information areas such as the originating tape drive, it assures the proper reel labeling.

The automatic tape scratch control feature returns volumes to the scratch pool as soon as it is feasible. There are several optional tape release controls available to the user, for example:

(1) The tapes are automatically released at a specified date,

(2) A tape is released to scratch when it is no longer found in an operating system data set catalog,

(3) The tape is released to scratch when it meets both conditions (1) and (2),

(4) The tapes can be released at any desired time without regard to conditions (1), (2), or (3).

Once a tape is released for any reasons whatsoever, its data set entry is removed from the master file either automatically or through a command.

The TMS-III performs most of its functions on a continuing basis without the necessity for user intervention. It automatically checks to make certain if a tape is in a scratch mode. If it is, then the tape can be used as desired after the master file is updated to its new status. The updated tape information includes its read/write error statistics. Importantly, TMS-III maintains sufficient background information on each processed tape so that data can be recovered in the event of a data loss.

Data center disasters can be minimized because TMS-III schedules and tracks off-site movement and storage of all critical tape data sets. 
It is possible to request the following output information from a system under TMS-III control:

- When will a particular tape volume expire?

- Which tape volumes have been or will be automatically scratched?

- What is the storage status of tapes; i.e., where are they located other than in the primary operating area?

- A read out of the master file contents.

- Which scratched tapes are to be certified and cleaned?

It is recommended by TMS-III that tapes should be cleaned after every 15 uses and evaluated/certified after every third cleaning (see sec. 1.14).

TMS-III offers a users selectable gummed label feature which gives positive external identification for all specified tape volumes.

\subsection{Surface Analysis System (SAS-I) 31}

SAS-I [36] is a specialized software utility program that is capable of simultaneously testing (evaluating) (see sec. 3.2.3) one to nine tapes online at normal tape speeds on the system tape drives. It certifies new or scratch tapes by recording them with a special character set which can defeat one or two bit error recovery; it can also certify data files by reading (READ ONLY) the existing data non-destructively and examining records and fields for bad spots. The certification is performed prior to actual tape use, therefore eliminating expensive job aborts.

SAS-I provides a permanent record in the form of a summary report for each tape which has been tested. These sum mary reports include:

- The number of temporary and permanent errors encountered.

- The number of write retries attempted in each error area.

- The length of the permanent errors and their approximate locations on the tape.

SAS-I also issues data check messages; i.e., abort messages to the console. This is done if, typically, 16 unsuccessful write retries occur on 16 consecutive locations during a test run. A different tape abort threshold, however, can be specified by the user if desired. The user may also specify the number of errors for which the tape shall be cleaned. Therefore, SAS-I may be used to schedule tapes for cleaning based on error counts rather than on fixed time intervals.

SAS-I can be used effectively in a scheduled maintenance program for monitoring the condition of tapes in long-term archives. It is recommended that these archival tapes be certified at least once per year (see sec. 2.1) using the READ ONLY function.

SAS-I can also be used to test the condition of the system tape drives. This is performed by recording the special character set on a known good tape on each of the transports under test, and then recording the total number of errors produced by each unit. For example, if eight to ten of the transports produce very few errors on this tape and the other two machines produce a large quantity of errors, then these latter two machines should be inspected and serviced prior to further use.

$\overline{31 \text { SAS-I is the author's }}$ designation for this system for convenience in presentation. 


\section{Clean Rooms and Clean Room Operations Guidelines}

The care and handling of magnetic storage media in research, calibration, manufacturing, and test facilities of ten require work areas in which airborne contamination is controlled. These areas known as clean rooms are described in Federal Standard No. 209A (August 10, 1966) entitled "Clean Room and Work Station Requirements, Controlled Environment."

\subsection{Description of Clean Rooms}

The standard defines air cleanliness classes based upon the maximum number of airborne particles of a particular size in a specified volume of space. For example, there are two particle size classes $0.5 \mu \mathrm{m}(20 \mu \mathrm{in})$ or larger and $5.0 \mu \mathrm{m}(200 \mu \mathrm{in})$ or larger, and there are three cleanliness classes: Class 100, Class 10,000, and Class 100,000. Class 100, which is the cleanest standard environment, is defined as an area which yields a maximum airborne particle count not to exceed $3.5 \times 10^{3}$ particles per $\mathrm{m}^{3}$ (100 particles per $\mathrm{ft}^{3}$ ) of particle size of $0.5 \mu \mathrm{m}$ (20 $\mu \mathrm{in}$ ) and larger. A Class 10,000 is defined as an area which yields a maximum particle count of $3.5 \times 10^{5}$ particles per $\mathrm{m}^{3}\left(1 \times 10^{4}\right.$ particles per $\left.\mathrm{ft}^{3}\right)$ of a particle size of $0.5 \mu \mathrm{m}(20 \mu \mathrm{in})$ and larger, or $2.3 \times 10^{3}$ particles per $\mathrm{m}^{3}$ (65 particles per $\mathrm{ft}^{3}$ ) of a particle size of $5.0 \mu \mathrm{m}(200 \mu \mathrm{in})$ and larger. Class 100,000 is defined as an area which yields a maximum particle count of $3.5 \times 10^{6}$ particles per $\mathrm{m}^{3}\left(1 \times 10^{5}\right.$ particles per $\mathrm{ft}^{3}$ ) of a particle size of $0.5 \mu \mathrm{m}(20 \mu \mathrm{in})$ or larger or $2.5 \times 10^{4}$ particles per $\mathrm{m}^{3}$ (700 particles per $\mathrm{ft}^{3}$ ) of a particle size of $5.0 \mu \mathrm{m}(200 \mu \mathrm{in})$ and larger.

Equipment employing light scattering principles are used (see ASTM F50) 32 for counting particles with sizes of $0.5 \mu \mathrm{m}(20 \mu \mathrm{in})$ and larger. For counting particles with sizes of $5.0 \mu \mathrm{m}(200 \mu \mathrm{in})$ and larger, microscopic counting of particles which have been collected on a membrane filter through which a sample of air has been drawn may be used (see ASTM F 25). 33

\section{Clean rooms fall into the following categories:}

(a) Non-laminar airflow types in which the airflow patterns and velocities are not uniform.

(b) Laminar airflow types in which the entire body of air within a confined area moves with uniform velocity along parallel flow lines with a minimum of eddies.

A conventional clean room uses non-laminar airflow design and makes use of highly filtered and conditioned air brought into the room area through individual diffusers located in the ceiling. It exhausts the air through return ducts located near the floor around the periphery of the room. Emphasis is placed on limiting the amount of contamination introduced into the air in the room by controlling the personnel, operations, and materials inside the facility. Personnel are required to wear low

32American Society for Testing and Materials (ASTM) F50: Method of Test for Continuous Counting and Sizing of Airborne Particles in Dust Contrólled Areas by the Light Scattering Principle (for Electronic and Similar Applications).

33ASTM F 25: Method for Sizing and Counting Airborne Particulate Contamination Clean Rooms and Other Dust Controlled Areas Designed for Electronic and Similar Applications. 
particle shedding type garments (see Guidelines at the end of this section), and all personnel and materials must be cleaned before introduction into the area. Removal of contamination is performed through careful maintenance and janitorial service.

A laminar type of clean room utilizes highly filtered and conditioned air which enters the room and flows towards the work area through a filter bank; this bank constitutes either an entire wall or the ceiling of the room. The air is then exhausted through an opposing parallel surface facing the air inlet filter bank. The air moves through the room in a laminar flow fashion, thus making only a single pass through any given area of the room. This laminar airflow stream carries (out of the room) both contamination which has been brought into the clean room on personnel and equipment, and airborne contamination generated by operations in the room. Contamination generated in localized areas of the room is isolated from other areas by the striations of the laminar airflow. Emphasis is placed on performing critical work in the regions of undisturbed clean airflow from the incoming air surface. Personnel restrictions, equipment cleanup, and operational limitations are minimized in a properly organized laminar flow room.

The laminar flow clean room is considered to be at the state-of-the-art in present day clean room technology and is the most desirable (and generally most expensive) type. Either type of clean room, both laminar flow and non-laminar flow, employ High Efficiency Particulate Air Filter Units (HEPA's); these are referred to as absolute filters. Some of these HEPA's are designed to remove up to $99.97 \%$ of the defined particles from the airstream and are very costly components in any clean room system.

When it is neither necessary nor economically feasible to construct a full sized Class 100 clean room, it is possible to construct small individual laminar flow, clean work stations at specific locations where specialized separate tests or measurements are performed. The exhausting from and the positioning of the work stations depend upon the operation being performed, the equipment involved, and the test or measurement residue. For example, operations that produce heat, dirt, or toxic exhausts should absolutely be ducted out of the laboratory environment. There are other tests or measurements which will not affect the ambient room particle count or the $\mathrm{T}-\mathrm{H}$ environment and will not require separate exhausting.

Full size clean rooms and clean work stations are constructed in both vertical (ceiling-to-floor) and horizontal (wall-to-wall) airflow configurations. Since many normal work areas and laboratories are not sufficiently air-tight to be made directly into clean rooms, room-within-a-room type of modular clean room configurations are available which are installed as integral units into these original work areas. There are numerous accessories available for use with clean rooms [10],[11]. Some examples are, parts pass-through chambers, through which items are transferred into the clean room proper without affecting the clean level integrity, and air showers and air locks through which the personnel enter the clean room area without introducing external contaminants.

In addition, there are individual clean work areas which are specialized for particular functions such as ultrasonic cleaning, medical research, photoresist, encapsulating and production assembly, and inspection. 
Airborne particle concentrations should be measured at representative locations in the clean room or work station. It is necessary to recognize that the differences between non-laminar and laminar airflow systems lead to different measuring techniques. For example, contamination generated in non-laminar flow rooms or work stations generally tends to be diffused over the entire work area, thus airborne particle counts will be fairly uniform through the whole work area. In laminar flow rooms, however, any airborne contamination released into the work area will follow the airstream path towards the exit; therefore, contamination levels in these rooms can vary from a Class 100 level at an air entry location to a much higher contamination level downstream of the dirtiest operation. Thus in a laminar flow facility, the sample of air should be taken in the area of interest. Also, the particle count should be taken at work height level and in the general work activity areas.

\subsection{Various Media Clean Rooms in Industry and Government}

The following information was obtained from various industry and Government magnetic media testing facilities that employed clean work areas. The inquiry was undertaken at the outset of the ICST Computer Storage Media program in order to determine the characteristics which are required for a facility which was to be used for the calibration and measurement of magnetic media Standard Reference Materials. It is important to note that most media organizations felt that temperature and humidity controls were generally more important than high cleanliness levels at their installations.

\section{(1) Government Agency A}

This organization designed its own clean room which was then constructed by a general contracting firm. The 99.97\% HEPA filters were used, and emphasis was placed upon maintaining the following temperatures and humidities:

Temperature: $21^{\circ} \mathrm{C}$ to $26.6^{\circ} \mathrm{C}\left(70^{\circ} \mathrm{F}\right.$ to $\left.80^{\circ} \mathrm{F}\right)$

Relative Humidity: $50 \% \pm 10 \%$

The clean room floor was raised and was made of an epoxy material. The walls were aluminum clad over plywood. These features produced shedfree surfaces. It was felt that asbestos floor tiles tended to abrade and add contamination into the environment. The raised floor was used in order to facilitate laminar airflow delivery to various work positions by individual ducts as well as to hide power cables. The room was employed for in-house experimentation and magnetic tape acceptance testing.

(2) Government Agency B

(a) This organization maintained a magnetic tape measurements room which was "semi-clean." It contained two continuously running precipitrons. There was no "through" traffic in this room and entry was restricted to only those who had to operate in the area. All personnel wore nylon boots and coats. Temperature and humidity was maintained within the following controlled limits:

Temperature: $21^{\circ} \mathrm{C}$ to $26.6^{\circ} \mathrm{C}\left(70^{\circ} \mathrm{F}\right.$ to $\left.80^{\circ} \mathrm{F}\right)$

Relative Humidity: $50 \%+3 \%$ 
(b) Agency $\mathrm{B}$ also maintained a magnetic tape acceptance testing area which was a Class 100 laminar flow room. It had an anteroom and an. air shower arrangement. Its environment was maintained at the same temperature-humidity values as the measurements room above.

(3) Magnetic Tape Manufacturer A

This was a large, established magnetic tape manufacturing organization whose original clean room facility was built before the advent of cleanliness standards and was not correlated to a specific Federal Class. A particle count program showed that large variations in count were caused by a simple movement of personnel in the clean room. Additional tests showed that the particle count had little effect on the production of signal dropouts; i.e., a large increase in particle count had produced a very small change in signal dropouts. The prevailing opinion was that precise temperature and humidity controls were a more critical requirement than extreme cleanliness levels. The company also found that the results of tests which were run in the summer and the winter differed considerably until strict $\mathrm{T}-\mathrm{H}$ controls were instituted. However, it should be noted, as expected, that as the recorded test densities increased, the effect of dust particles on dropouts became more serious (see sec. 8.1-eg(4)).

This clean room installation had a complete change of air each minute and encouraged clean working habits among the personnel who were required to wear white, lint-free coats but no hats or boots. it was noted that one useful purpose for the white coats was psychological in nature since they tended to encourage cleanliness by their presence. The environment in the laboratory was maintained under the following conditions:

(a) Temperature: $22^{\circ} \mathrm{C}$ to $24^{\circ} \mathrm{C}\left(71^{\circ} \mathrm{F}\right.$ to $\left.75^{\circ} \mathrm{F}\right)$

(b) Relative humidity: 45\% (nominal value)

(c) Filtering was accomplished through 99.7\% absolute filter types (this was less than a Class 100 requirement). The air was prefiltered before entering the absolute HEPA filters.

(d) Positive pressurization was produced by an airflow of approximately $0.14 \mathrm{~m}^{3} / \mathrm{s}\left(5 \mathrm{ft}^{3} / \mathrm{s}\right)$ to $0.2 \mathrm{~m}^{3} / \mathrm{s}\left(6.7 \mathrm{ft}^{3} / \mathrm{s}\right)$.

(e) A plenum ceiling discharged the air into the room.

(f) Clean clothes were worn by operating personnel, but there was no air shower arrangement at the entrance to the room.

(g) The room had a terrazzo (epoxy with chips) type floor. 
This company tested large quantities of bulk magnetic tape. It originally attempted to maintain a Class 100 clean room environment but was unsuccessful due to the tape material conveyor system that was used. At best, it was able to achieve a Class 1,000 room. The company also felt that temperature and humidity controls were very important, while extreme dust control was relatively unimportant. It noted that NBS reference tape measurements would be of those tape properties in which dust would not affect the results and that anomalies due to an occasional dropout due to dust could easily be removed from the final results; this eventually proved to be correct. However, they further noted that if dropout testing is done in quantity, then higher degrees of cleanness, such as can be achieved with clean work stations, would be in order.

(5) Magnetic Tape Manuf acturer C

This organization noted that: (1) a tape measurement laboratory should be restricted to only those personnel that are actually working in it, with no through traffic permitted; (2) a very clean working arrangement could be obtained by having a laminar airflow over both the operator and the working surface, with the machine heat and residue being exhausted out of the laboratory through a duct system; and (3) the minimum requirements for the laboratory were:

(a) Construct the room with non-shedding materials.

(b) Use positive air pressure.

(c) Lighting fixtures should be cleanable and have as few ledges and dust catching surf aces as possible.

(d) Temperature and humidity control was absolutely necessary.

(e) Special clothes were not important.

(6) Tape Manufacturer D

This organization had a clean room which did not achieve a Class 100 level. They had an air-lock en trance, clean monofilament garments, hair nets but no booties. For the volume of traffic that they carried, a Class 100 room was not feasible, since in fact such a clean room should have no traffic. They used absolute HEPA filters (99\% plus, particle removal rate), controlled temperature and humidity, and moved a large volume of air in a non-laminar flow pattern. They also maintained positive room air pressure. In their new plant, they used 99.97\% HEPA filtering in a laminar flow system in those operating areas where the magnetic coatings were still in the liquid state, and absolute cleanliness was required. At the time of this inquiry, this company used small, individual laminar flow stations for some of their research and testing operations.

(7) Miscellaneous

It has been reported by one media manufacturing organization that their operating personnel experienced psychological effects which were caused by a feeling of isolation when working in their fully enclosed Class 100 
clean room. The organization subsequently changed to a (approximate) Class 10,000 clean room with cleaner individual work stations. This permitted a greater personnel traffic flow and relieved the isolation problem. It has been reported by one organization that their research and development engineers generally did not follow all of the very strict procedures which are required when working in a full Class 100 room, and that they performed considerably better at individual laminar flow stations. However, it should be noted that in some technologies a full Class 100 clean room is absolutely required. For example, a Class 100 clean room is needed when working with materials and devices such as semiconductor materials and delicate gyroscopes whose characteristics can be damaged by contamination with foreign matter. It was noted that individual clean work stations of Class 100 levels when used, should be immersed in areas which themselves are are of Class 1,000, if possible.

\subsection{General Guidelines for all Types of Clean Rooms and Clean Work Stations 34}

(1) Garments specified as applicable to a given facility must be worn when entering the area; for example, lint-free smocks and coveralls.

(2) Neither smoking nor eating should be permitted in the clean room area.

(3) Head covering, which covers the entire head, should be required to avoid component contamination by loose bits of hair or loose bits of skin flakes.

(4) No cosmetics shall be worn by any worker while in any clean area. This shall include: rouge, lipstick, eye shadow and eyebrow pencil, mascara, false eye lashes, fingernail polish, hair spray, and the heavy use of an aerosol deodorant.

(5) Limit paper entering area. There are special nonshedding papers for necessary records or paperwork which are approved for clean room applications.

(6) Use only ballpoint pens for writing. Lead pencils and erasers should not be permitted in clean room area.

(7) The use of paper or fabric towels should be forbidden. Wash rooms should have electrically powered, warm air dryers.

(8) Hand lotions, creams, or soap containing lanolin to tighten skin particles should be used, as appropriate.

(9) Solvent contact with hands should be avoided, as many solvents remove natural skin oils causing excessive "skin peeling" or flaking.

(10) Lint-free gloves, finger cots, tweezers, or other handling methods and equipment should be used while working with or handling sensitive parts, to avoid contamination of those parts by loose skin or natural skin oils.

(11) Clean all equipment before entry into area by dusting, vacuuming, washing, or by suitable means, as best suited to equipment involved.

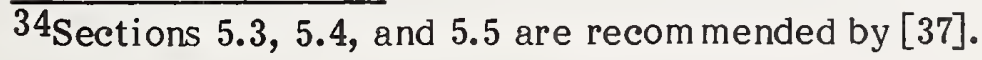


(12) Gloves or finger cots should not be permitted to touch any item or surface that is not known to have been thoroughly cleaned. Specifically, they should not touch any part of the anatomy, covered or uncovered.

(13) No tool or part should be permitted to be placed on the bench or other surface until that surface has been cleaned with a clean lint-free, isopropanol dampened cloth. Other equally effective solvents, compatible with the surface, may be used if isopropanol is not available.

(14) No tool should be allowed to rest on the surface of the bench or work surface, but should be placed on a hardware cloth or perforated metal rack, to guard against contaminants which have settled on the work surface being transferred to the tool.

(15) All parts or material containers, racks, jigs, fixtures, and tools should be cleaned to the same level of cleanliness specified for the product being processed.

\subsection{Guidelines for Nonlaminar Airflow Clean Rooms and Clean Work Stations}

(1) Garments shall be pocketless, lint-free coveralls, with provisions for snugly fastening the neck, wrist, and ankles.

(2) The caps which must be worn, shall be of lint-free fabric, designed to cover the head and hair completely, except for the face--which shall include the eyes, nose, mouth, and chin.

(3) Shoes shall be cleaned and covered with a nonshedding boot type cover, or changed to approved clean room footwear. If special footwear is provided, they shall not be worn outside the clean room and dressing room.

(4) The use of compressed air, except in hoods specifically designed for that purpose, shall not be permitted. Any operator assigned to such a hood shall have special instructions for its proper use.

(5) Room maintenance, except for vacuuming by a properly garmented employee, shall be restricted to periods when no productive activity is being performed, and when any material or work in process is covered by an approved cover.

(6) Janitorial services shall be performed only by adequately trained and supervised personnel, each of which must be properly garbed.

(7) All equipment which must be brought into the clean room, shall first be thoroughly cleaned, and the use of the equipment in the room shall not generate any contaminants.

(8) Traffic into and within the clean room shall be restricted to authorized personnel and unnecessary movements by the personnel shall be minimized. 


\subsection{Guidelines for Laminar Airflow Clean Rooms and Clean Work Stations}

(1) Garmenting may vary with the type of operation being performed, but the minimum garmenting shall be a pocketless, lint-free smock, or coat, which must extend to a minimum of $0.4 \mathrm{~m}(1.25 \mathrm{ft})$ below the work surface. The collar and cuffs of the garment shall be equipped to be fastened at the neck and wrists.

(2) Head coverings shall be worn, and shall be capable of completely covering the hair area. If the operation requires the wearer to lean over the work, or move into the airstream between the filter bank and the work piece, the front, sides, and rear neck area shall also be covered.

(3) Special shoes, or shoe covers may not be necessary for vertical laminar airflow facilities, nor for horizontal airflow installations except when the work is being performed at a point less than $0.6 \mathrm{~m}(2 \mathrm{ft})$ from the floor.

(4) A face mask may be needed if an operator has a cold, or if the nose and mouth must be brought very close to the work piece, for work on miniature parts. 


\section{References}

[1] Gray, M. M. An assessment and forecast of adp in the federal government. Nat. Bur. Stand. (U.S.) Special Publication 500-79; August 1981. 134 p.

[2] Information Systems Planning Service. Data center security: disaster planning, backup, and recovery. International Data Corporation (IDC). Report ISPS-R81-01; March 1981. 93 p.

[3] Shaw, J. K.; Katzke, S. W. Executive guide to adp contingency planning. Nat. Bur. Stand. (U.S.) Special Publication 500-85; January 1982. 10 p.

[4] Dollar, C. M. Problems of magnetic recording in archival storage. Spring COMPCON Digest of Papers; 14th IEEE Computer Society International Conference; 1977. 3 p.

[5] Geller, S. B. Archival data storage. Datamation; October 1974. 4 p.

[6] Staff National Archives and Records Service. Recommended environmental conditions and handling procedures for magnetic tape (unpublished document); 1972. 21 p.

[7] Technassociates, Inc. A study of archival storage technology for machine-readable archives. NARS-GSA (Contract No. NA76-12). 1976. 80 p.

[8] Rosenkrantz, G. J. National archives mass storage requirements $-1975-1980$. IEEE Transactions on Magnetics, VOL. MAG-7, No. 4; December 1971. 5 p.

[9] McKenna, R. T. Digital recording reliability for information exchange application. American National Standards Institute - X3B1/651; June 1973. 24 p.

[10] Gold Book, Vol. 1. Electronic Design. Hayden Publishing Co., Inc., 50 essex Street, Rochelle Park, NJ 07662; 1981/1982. 1200 p.

[11] eem, Vol. 1. United Technical Publications, 645 Stewart Avenue, Garden City, NY 11530; 1981/1982. 2570 p.

[12] Product Catalogs

(a) U.C.I., Computer Supplies Specialist, Seven Woodside Avenue, Berwyn, PA 19312

(b) Inmac, 2465 Augustine Drive, Santa Clara, CA 95051

(c) Devoke Data Products, 3780 Fabian Way, Palo Alto, CA 94303

(d) Misco Inc., 404 Timber Lane, Marlboro, NJ 07746

[13] Geller, S. B. Erasing myths about magnetic media. Datamation; March 1976. 5 p. 
[14] Geller, S. B. The effects of magnetic fields on magnetic storage media used in computers. Nat. Bur. Stand. (U.S.) Technical Note 735; July 1972, 30 p.

[15] Tape Head Interface Committee; Kalil, F. ed. Magnetic tape recording for the eighties. NASA Reference Publication 1075; April 1982. 170 p.

[16] European Computer Manufacturers Association. Guidelines for magnetic tape handling. ECMA/TC17/80/7; July 1980. 7 p.

[17] Magnetic tape management. Computer-Link Corp.; 1979. 19 p.

[18] Eldridge, D. F. Causes of failure in magnetic tape. Memorex Monograph No. 3; May 1964. 4 p.

[19] Magnetic Products Division 3M. The handling and storage of computer tape. Computer Talk, Vol. 1, No. 1; 1968. 7 p.

[20 Rodriguez, J. A. An analysis of tape drive technology. Proceedings of the IEEE, Vol. 63, No. 8; August 1975. 7 p.

[21] Jorgensen, F. The complete handbook of magnetic recording. Chapter 14. Tab Books, Inc.; July 1980. 448 p.

[22] N. Bertram; A. Eshel. Recording media archival attributes (Magnetic). Rome Air Development Center. RADC-TR80-123; Aprii 1980. 112 p.

[23] Romei, L. Protecting the lifeblood of dp. Modern Office Procedures; March 1982. 3p.

[24] Heck, C. Magnetic materials and their applications. Chapter 17. Crane, Russack and Company, Inc.; 1974. 7 p.

[25] Geller S. B. Layaway, standby, and reactivation procedures for computer magnetic media. Proceedings of the Workshop on Control Systems for Munitions Plants. (Purdue University, West Lafayette, Ind.); September 1977. $11 \mathrm{p}$.

[26] Poland, W. B. Jr.; Prine, G. E.; Jones, T. L. Archival performance of NASA GSFC digital magnetic tape. American National Standards Institute X3B1/650; June 1973. 29 p.

[27] Noel, J. D. - Proj. Mgr. Effects of storage on digital magnetic tape. Phase II Report. Wolf Research and Development Corporation. NASA-GSFC Contract No. NAS-5-9756-113; July 1968. 47 p.

[28] Cuddihy, E. F. Aging of magnetic recording tape. IEEE Transactions on Magnetics, Vol. MAG-16, No. 4; July 1980. 11 p.

[29] Cuddihy, E. F. Hygroscopic properties of magnetic recording tape. IEEE Transactions on Magnetics, Vol. MAG-12, No. 2; March 1976. 9 p. 
[30] Bertram, H. N.; Cuddihy, E. F. Kinetics of the humid aging of magnetic recording tape. IEEE Transactions on Magnetics, Vol. MAG-18, No. 5; September 1982. 7 p.

[31] Mandle, J. Evaluating performance of digital magnetic tape. Memorex Monograph No. 5; April 1967. 8 p.

[32] Wardle, D. Why disk and tape management systems? Infosystems, Vol. 29, No. 4, Part 2; April 1982. 2 p.

[33] Auerbach Computer Technology Reports. Reference 680.6767.800. Auerbach Publishers Inc., Systems Sof tware, Vol. J-1; 1982. 2 p.

[34] Ibid., Ref erence 680.6740.500.

[35] Ibid., Reference 680.2040.800.

[36] Datapro 70. Reference 70E-528-02a. Datapro Research Corp., Sof tware Vol-3; May 1982. 2 p.

[37] Standard Clean Room Operational Guideline. Moore and Hanks Co., El Monte, CA 91733; 1975. 12 p.

[38] Carroll, J. F. Jr.; Gotham, R. C. The measurement of abrasiveness of magnetic tape. IEEE Transactions on Magnetics, Vol. MAG-2, No. 1; March 1966. 8 p.

[39] Christensen, R. M. Theory of viscoelasticity - an introduction. Academic Press Inc.; 1971. 245 p.

[40] Bate, G. Oxides for magnetic recording. Magnetic Oxides Part 2, Chapter 12. Craik, D. J. ed., John Wiley and Sons; 1975. 54 p.

[41] Mallinson, J. C. Tutorial review of magnetic recording. Proceedings 'of the IEEE, Vol. 64, No. 2; February 1976. 13 p. 


\section{Appendix A. Handling and Environmental Effects}

\subsection{Effects of Hydrolysis on Tapes}

Large variations in temperature and humidity which may be encountered by computer magnetic tapes in storage, in transit, or in uncontrolled environments, are among the principal causes for physical and chemical changes and eventual damage to the media. Secondary damage to the tapes can be caused by the distortion of the flanges on the plastic reels and/or the hubs under extreme environmental conditions.

Three of the major components of a computer magnetic tape (i.e., the mylar substrate, the polyurethane binder, and the backcoating) display hygroscopic properties. That is, they both absorb and desorb atmospheric moisture as a function of the relative humidity. In an unsealed area such as a computer center or large vault, the water content of the tape will come into equilibrium with the relative humidity of the atmosphere [29]. It has been found that a number of adverse reactions are related to both extreme or fluctuating humidity environments. These reactions include increased tape abrasivity, increased head wear, increased tape debris and associated error rates, gummy products which are produced by polyurethane binder degradation, and tape pack tension increases and decreases leading to tape cinching 35 or blocking. 36

Studies [28],[29],[30] using commercially available magnetic tapes, concluded that a primary cause of tape aging and degradation was the hydrolysis of their polyurethane binders. Hydrolysis is a double decomposition reaction in which water is one of the reacting substances. In the case of computer magnetic tapes, the hydrolysis process consists of atmospheric moisture plus ester combining to form carbolic acid and alcohol. Since the ester is required to maintain the crosslink structure in the polyurethane binder, the reaction tends to weaken the binder and cause tape binder shedding and deteriorated mechanical properties. Fortunately, this chemical reaction is a reversible process; that is, tape binder rejuvenation can be accomplished by storing the media in a proper temperature-humidity environment (see sec. 2.2.1).

In tests performed with tapes in four different temperature environments, it was found that from $30 \%$ to $100 \%$ relative humidity $(\mathrm{RH})$, the binders were degrading; while from $0 \%$ to $11 \% \mathrm{RH}$, the binders improved chemically. Hence, the tapes remain stable in an environment with relative humidities in the range of $11 \%$ to $30 \%$ $\mathrm{RH}$, while tape binder restoration or rejuvenation begins in relative humidities below $11 \% \mathrm{RH}$ and is best accomplished at $0 \% \mathrm{RH}$ (i.e., a dry gas environment). The hydrolysis process is accelerated by increases in temperature.

Most importantly, these studies have led to chemical theories, and test methods for predicting and extending the lifetimes of magnetic tapes. These lifetime predictions are not based solely upon their chronological ages but also upon their chemical ages which are a function of the hydrolysis cycles the tapes have already experienced. The studies have determined that an optimal archival storage environment is one which is tightly controlled to (see sec. 2.2 .1 and fig. 3.): $18.3^{\circ} \mathrm{C}$ (65F), $40 \% \mathrm{RH}$.

35 Tape cinching is the folding or creasing of the tape which is caused by tape slippage upon acceleration or deceleration.

36 Tape blocking is the adhesion of adjacent tape layers to each other. 
Another finding in these studies was that tapes which were sealed in a hermetic environment at a constant temperature tended to improve their chemical (binder) quality. This occurred regardless of the initial humidity at the time of the sealing. This finding is important, for example, with units such as sealed, spacecraft recorders.

\subsection{Effects of Humidity on Tape Abrasivity}

It was found [38] that the relative humidity had a pronounced effect on the abrasiveness (i.e., head wear tendencies) of a number of the tapes which were under investigation. For example, tapes which were abrasive at $50 \% \mathrm{RH}$ became virtually non-abrasive below 5\% RH. Tape friction was observed to rise even further with increasing humidity. Several observations were made as an outgrowth of this humidity dependence:

(a) A long tape loop which was preconditioned in a 50\% $\mathrm{RH}$ environment and maintained in that condition during a test, exhibited approximately $5 \%$ of its expected abrasivness when the vicinity surrounding the area where the tape contacted a $12.7 \mathrm{~mm}$ ( $0.50 \mathrm{in})$ diameter metal test rod was flooded with dry air. This vicinity included less than $25.4 \mathrm{~mm}(1.0 \mathrm{in})$ of tape length.

(b) The tapes used with various types of metal rods in a "rod-wear" test which substituted these rods for the actual heads, displayed a metal dependent abrasivity response under fixed humidity conditions. For example, in tests using a group of equally polished metal rods made of annealed and unannealed high permeability alloys, unannealed brass, aluminum, and copper, it was found that the high permeability alloys produced a sharp increase in tape abrasiveness at $50 \% \mathrm{RH}$, while aluminum and copper produced very little response. The type of brass which was used produced approximately one half of the tape abrasiveness as compared to the high permeability alloys. Since aluminum and copper are used in interchannel shields and spacers in some read/write heads, it was surmised that this may explain why high permeability pole-pieces and other head-surface materials are found to wear equally in some installations, while in others the pole-pieces are channeled out due to a higher wear rate.

(c) Later test results [29] did not display a direct correlation bet ween the hygroscopic properties of the magnetic tapes and their abrasive wear patterns. However, it was felt that there still was a possibility that such a relationship existed. It was further suggested that the observed breakpoint at $40 \%$ to $45 \% \mathrm{RH}$ at which there was a sharp increase in measured abrasiveness was in fact due to the materials used for the construction of the recording heads; the tapes were involved mainly in determining the magnitude of the abrasive wear.

Two possible mechanisms were hypothesized relative to the deposition of the tape binder materials onto the heads:

(1) Tapes which were formulated with hard binders tended to wear the head surfaces with the preceding $40 \%$ to $45 \% \mathrm{RH}$ abrasivity 
breakpoint characteristic. Increased humidity led to increased head wear while decreasing humidity led to a reduction in tape flexibility and an increase in static charges on the tape (see sec. 1.10-(11)).

(2) Tapes which were formulated with low friction, soft binders tended to have their own surfaces worn by the heads with little to no head wear. The wear products from the tape were either gummy or powdery. This was thought to be a function of the relative humidity and the hygroscopic characteristics of the tape coating. It was surmised that tapes which absorbed more atmospheric vapor would tend to shed gum mier coating products.

(d) An adverse reaction known as "brown-stain" occurs at low humidity levels (i.e., below $35 \% \mathrm{RH}$ ). It manifests itself as a discoloration of the recording head surface by the media. Brown-stain cannot be removed with ordinary cleaning solvents; instead it must be polished off with an abrasive media. The stain causes a head-to-tape spacing loss (sec. 8.1-eq(4)) which is most detrimental for shorter wavelength signals.

\subsection{Effects of Viscoelasticity on the Tape Pack}

While an elastic material is capable of storing mechanical energy under stress without energy dissipation, a viscoelastic magnetic tape base material such as polyethylene terephthalate (mylar) both stores and dissipates energy in response to winding stresses. This energy change is performed through processes known as creep and stress relaxation which differentiates this material from a purely elastic material. An unusual characteristic of a viscoelastic material is its ability to continue to alter its dimensions (i.e., plastic or cold flow) in response to previously applied tensile stresses which have already been superseded by different stress values. This phenomenon is referred to as the "memory" of the material [39] and explains why the windings in a reel of tape will of ten develop a different tension and pressure pattern after it has been stored for some time even in a controlled environment. It may, in fact, have cold flowed in response to the winding stresses that it experienced at some earlier time, possibly at the time of manuf acture.

Therefore, a stored magnetic tape is never in a truly inert state because of its viscoelastic nature. That is, the polymeric tape materials cold flow continuously in order to relieve the wound in initial tensile stresses in the tape pack; this results in a reduction of the layer-to-layer frictional forces at various regions in the pack. Hence, a computer tape whose pack is initially firm and smooth with no protruding windings, can develop loose windings after a period of storage; this can lead to tape cinching upon acceleration or deceleration. The interaction between the tape winding tension pattern prior to storage, and the temperature and humidity variations which occur during the same storage period is a source of eventual tape problems.

\subsection{Effects of Temperature, Humidity, and Winding on the Tape Pack}

Constant tension tape winding is a prevalent method used in tape drives and other tape handlers at the present time. The initial pressures experienced by incividual layers wound onto a tape pack at a constant tension (i.e., constant tensile stress) form a continuum ranging initially from almost zero pounds per square inch at the 
outermost layers to several thousand pounds per square inch on layers near the hub. 37 These inner pressures can increase significantly if the tape is stored at a higher temperature than its initial winding temperature, and the hub coefficient of thermal expansion is greater than that of the tape pack. In addition, if the tape has been initially wound in a higher relative humidity environment than the storage humidity, then the tape will desorb the moisture and shrink; this increases the tape pack pressures, especially in the absence of equal hygroscopic contraction of the hub [22] These increasing pressures can cause various types of tape damage. For example, tapes which are stored in environments in which large temperature and humidity variations occur, can develop extreme tape pack pressures which can result in layer-to-layer adhesion (i.e., blocking) of the tape layers, and in cases of extreme blocking, tape coating can be torn from the base material as it is unwound. Layer-to-layer adhesion increases with the time the tape is stored and with the number and magnitude of temperature-humidity cycles during storage.

Other deleterious effects caused by temperature-humidity variations are the longitudinal and transverse distortions of the tape. The distortions take on several identifiable forms, among which are cupping and skewing. Cupping is a transverse curving or bowing of the tape from edge-to-edge. It manifests itself when there are differences in the thermal and hygroscopic coefficients of expansion between the coating containing the magnetic particles and the mylar base material. Skewing is a longitudinal curvature along the tape edges which is manifested as a departure from a straight flat line drawn along the tape center. While cupping can cause variations in track-to-track signal amplitudes, skew causes a time misalignment of the characters derived from each of the head tracks. Besides resulting from temperature and humidity effects, skew can also be caused by poor web tension during the manufacturing tape slitting process, by transport guide misalignment, and by poor tape winding (for example, high speed rewinding) during operation prior to storage. Sometimes, a skewed tape can be corrected by winding and rewinding the tape a number of times at normal speeds onto a precision reel under careful tension control. The tape is then allowed to relax for at least one day in the environment in which the reading will be performed. It is beneficial to rewind the tape again several times before reading.

Cinching can be caused by winding tension patterns which are produced by the tape transport mechanism. It occurs as the result of a tape winding pattern in which the direction of the tension vector in the tape windings will reverse somewhere within the tape pack from an inward compressive tension, which shortens the tape near the hub, to an outward (expansive) tension as the cold flow process continues. In the region of the tape pack where the tension reversal takes place, there are a number of tape layers under zero tension in which the layer-to-layer friction of the tape is greatly diminished. Hence, when the reel is mounted onto a tape transport and accelerated or decelerated rapidly, the tape pack acts like individual concentric cylinders in which the innermost portions of the reel may accelerate or decelerate more rapidly than the outermost portions. Tape slippage occurs and causes a cinching or creasing of the tape between these regions (cylinders); this damages both the media and the data over a large region. There are commercial winders available known as programmed tension winders which adjust the winding tensions so that there is reduced compression in the inner tape layers near the hub; this eliminates

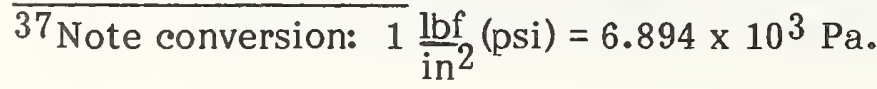


the zero tension layers in the tape pack and the tendency for tape slippage to occur. There is also a tendency for tapes to cinch if there is an insufficient initial winding tension which has produced a loose or sloppy tape pack.

Tape pack distortion can be caused at low temperatures by the shrinking of plastic reel hubs whose thermal properties are different from those of the polymeric tape pack material. This causes the tape pack to loosen and the tape to cinch or buckle (i.e., form a non-circular pack). Note that since the thermal coefficient of expansion of aluminum is nearly equal to that of the polyester base film, therefore, aluminum hubs can minimize this effect. The dimensions of the reel hub and the compressibility of the hub material also have an effect on the characteristics of a computer tape pack which is held in long-term storage. It has been found that [18]:

(a) compressive forces on the hub can be reduced by keeping the ratio of the outside pack diameter to the reel hub diameter to a value less than three, and

(b) it is advantageous to have a large, incompressible hub because the more incompressible the hub the more the tape pack resists distortion (buckling) and tends to retain its cylindrical symmetry; it will also retain its initial winding tension values over a longer period in storage.

The following are effects of elevated temperatures on a tape pack [19]:

- At $121^{\circ} \mathrm{C}\left(250^{\circ} \mathrm{F}\right)$, the substrate (mylar) becomes distorted.

- At $160^{\circ} \mathrm{C}\left(320^{\circ} \mathrm{F}\right)$, the substrate and the coating become soft; blocking (i.e., layer-to-layer adhesion) begins in the tape pack windings.

- At $288^{\circ} \mathrm{C}\left(550^{\circ} \mathrm{F}\right)$, the substrate and the coating become darker and brittle.

- At $538^{\circ} \mathrm{C}\left(1000^{\circ} \mathrm{F}\right)$, the substrate and the binder become charred.

In addition to the effects of temperature on the tape media, there are temperature problems which are unique to the read/write heads themselves. These problems are caused by the differences in the temperature coefficients of expansion of the various delicate head components and manifest themselves as changes in the head signal response.

It is interesting to note that under normal winding stresses, the length of a $731 \mathrm{~m}$ $(2400 \mathrm{ft})$ computer tape will increase by approximately $0.305 \mathrm{~m}(1 \mathrm{ft}) \mathrm{for}$ each $5.6^{\circ} \mathrm{C}$ $\left(10^{\circ} \mathrm{F}\right)$ change in temperature or $10 \%$ change in relative humidity [7].

\subsection{Signal Rise Effect}

It has been noted that many different computer tapes display a steady rise in signal amplitude as the tape is read from end-to-end (BOT to EOT) with the signal level increasing most rapidly as the reel hub is approached. It appears that this signal rise or "tilt" effect is caused by the increasing tape pack pressures through their smoothing or calendering effects on the tape surface. This smoothing effect improves the head-to-tape contact continuously as a function of the increasing winding pressures proceeding from the BOT to the EOT along the tape surface. In addition, the amount of signal rise has been found to be signal wavelength dependent; this further validates the head-to-tape spacing (through smoothing) 
theory as defined by the spacing loss equation (sec. 8.1-eq(4)). The percentage of tilt also found to be a function of the read head characteristics during some of the tests.

It is suggested as an experimental study, that the winding pressure and tension characteristics of a tape transport be investigated through the use of specially produced, uncalendered, non-backcoated, soft-binder computer tape which will be responsive to the surface smoothing mechanism after a shorter storage period. It has been observed at the ICST Computer Storage facility, that tapes with soft binders which were stored for long periods of time displayed nonlinear signal level responses along their length. This appears to indicate that different pressure profiles developed in storage, assuming that the smoothing mechanism theory is valid. Also note the friction tab measurement technique in section 2.2.3.

It has also been observed, that the normal signal level rise from the BOT to the EOT on a tape could be reversed so as to rise from the EOT to the BOT, if the tape is wound in the forward direction and then stored without rewinding, with the EOT remaining in the outermost, low pressure, pack position. 


\section{Appendix B. Computer Magnetic Tape Recording and Materials}

\subsection{Computer Magnetic Tape Recording}

(a) All reproduction of signals from present day computer tapes and disks is done inductively. That is, the reading of the signal from a tape surface is done by a transducer or head which responds only to the rate of change of magnetic flux $\phi$ issuing from the surface. Most present day computer tape recording is in the form of "longitudinal" recording. In longitudinal recording, the principal direction of magnetization is in the plane of the surface and parallel to the direction of surface motion. Considerable research is presently underway in the area of perpendicular recording.

(b) The basic inductive magnetic recording process proceeds as follows: during the recording process, a magnetic field fringes outward from the gap of a magnetic tape recording head. This magnetic field varies in both intensity and direction dependent upon the current in the recording head coils. This current is in turn controlled by an electronic system which converts information bits to reversals in recording head current. The fringing field from the recording head gap structure permeates the oxide coating of the medium and leaves permanently magnetized areas in the form of transition regions from which the tape surface fringing flux emanates. A transition region is an area of the tape surface over which the recorded magnetic flux reverses its polarity in response to the recording signal. The read or reproduce head then senses these surface fringing flux fields inductively and produces output voltage pulses at each bit position; these voltage pulses represent the stored information bits. There is no output from the read head except at those tape surface areas from which the fringing fields emanate. The signal strength derived from the medium depends upon the gradient of the flux as follows:

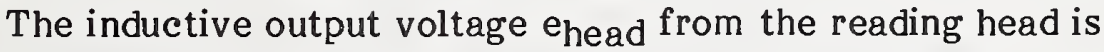

$\mathrm{e}_{\text {head }}=\frac{\mathrm{d} \phi}{\mathrm{dt}}=\frac{\mathrm{d} \phi}{\mathrm{dx}} \quad \frac{\mathrm{dx}}{\mathrm{dt}}=\mathrm{v} \frac{\mathrm{d} \phi}{\mathrm{dx}}$

Where $v=$ the relative reproducing head-to-medium velocity and,

$\frac{d \phi}{d x}$ the rate of change of flux $\phi$ with respect to the distance $x$ along the medium surface, therefore the faster the medium moves relative to the reproducing head, and the more rapidly the flux varies with distance (over a transition region), then the greater the output signal strength induced by each bit or transition region on the tape.

(c) High density recording requires fast reversals in the direction of magnetization in the media surface. As indicated, each reversal produces a transition region, and typically one or two transition regions represent an information bit. The faster each reversal of magnetization, then the narrower each associated transition region, and therefore the more magnetization changes or bits per unit length of surface (i.e., bit density) a media is capable of producing and storing. 
(d) Factors which influence the bit density and the signal strength obtainable from the magnetic media are:

(1) The thickness $t$ of the coating on the surface of the storage medium.

(2) The coercivity $\mathrm{H}_{c}$ of the magnetic oxide particles.

The ability of a magnetic material to resist the erasure of its recorded information is dependent upon a quality known as coercivity which is measured in units of amperes per meter (oersteds). The higher the coercivity of a magnetic medium the more difficult it is either to record onto or to erase information from its magnetic surface. In order to erase the recorded signal completely, it is necessary that the strength of the erasing field is greater in value than the coercivity of the magnetic material.

In order to maximize the bit density, the foregoing conditions are related as follows. The minimum width of a reproduced pulse $\mathrm{P}_{\mathrm{W}}$ at its $50 \%$ points is:

$\mathrm{P}_{\mathrm{W}} \cong\left(\frac{\mathrm{t}}{\mathrm{H}_{\mathrm{c}}}\right)^{\mathrm{n}}$ where $\mathrm{n}=1 / 2$ to 1 .

This equation shows that in order to achieve high bit densities the coercivity of the medium should be as high as possible and its coating thickness $\mathrm{t}$ as small as possible. In addition, high coercivity $\mathrm{H}_{c}$ opposes the tendency of internal demagnetization fields $\mathrm{H}_{\mathrm{d}}$ which form in the transition regions from forcing these regions to widen, and hence reduce and limit the bit density capability of the medium.

The coercivity $\mathrm{H}_{\mathrm{c}}$ is controlled through the processing of the magnetic materials both physically and chemically. $\mathrm{H}_{\mathrm{c}}$ can be made very high say, $80,000 \mathrm{~A} / \mathrm{m}$ (1000 Oe); however, as $\mathrm{H}_{\mathrm{c}}$ increases, the recording or writing head power must also be increased. This can cause head (pole tip) saturation problems, and can deteriorate the sharpness of the writing head fields; this affects the bit density capability of the system. Typical coercivities for present day computer tapes are approximately $22,000 \mathrm{~A} / \mathrm{m}(275 \mathrm{Oe})$ to $30,000 \mathrm{~A} / \mathrm{m}(375 \mathrm{Oe})$ although coercivities on the order of $48,000 \mathrm{~A} / \mathrm{m}(600 \mathrm{Oe})$ are available in some new high energy tapes. The thickness $t$ of the magnetic coating should be made as thin as possible consistent with an acceptable output signal, good surface finish, uniformity of coating, and good wear characteristics. Signals that are recorded in the lower strata of a thick coating combine with the sharper pulses produced near to the tape surface and tend to broaden the overall signal. This reduces the ability of the medium to produce many sharp pulses per unit length along the tape's magnetic surface coating.

Additional factors which affect the bit density capability and the signal strength are:

(3) The spacing between the reading and writing head (transducer) and the storage media. 
(4) The shape or gradient $\frac{\mathrm{dH}}{\mathrm{dx}}$ of the magnetic field which extends or fringes from the writing $d x$ head; where $x$ is the longitudinal component of the head's $H$ field along the surface of the tape. $H$ is the applied recording field strength in the $\mathrm{x}$ direction.

(5) The magnetic characteristics of the storage medium, $\frac{d I}{d H}$ where I is the magnetization of the medium. $\mathrm{dI}$ is the slope $\mathrm{dH}^{\mathrm{d}}$ of the $\mathrm{I}-\mathrm{H}$ hysteresis loop of the magnetic $\overline{\mathrm{dH}}$ material as it passes through the $\mathrm{H}$ axis. A steep slope produces sharply defined switching field characteristics.

(6) The following equation is derived from items (4) and (5) above [40]:

$\frac{\mathrm{dI}}{\mathrm{dH}} \times \frac{\mathrm{dH}}{\mathrm{dx}}=\frac{\mathrm{dI}}{\mathrm{dx}}$

where:

$\mathrm{dI}$ is the change in the intensity of magnetization as a function $\overline{\mathrm{dx}}$ of distance along the tape's magnetic surface: the greater $\frac{\mathrm{dI}}{\mathrm{dx}}$ the greater the inductive (signal) voltage produced per $\overline{d x}$ information bit.

(7) The crowding of adjacent signal bits at high densities, causes read signal peak shif ting; this can lead to data losses if the shift is greater than a bit cell.

(e) An important signal loss effect is caused by the head-to-media spacing. The height of a flying head above a disk surface or the average distance of a magnetic tape reproducing head from the tape surface both cause this loss. Head-to-tape spacing is caused by the roughness of the magnetic surface and exists even though the head and tape operate in apparent contact with each other. Modern computer magnetic tapes are being produced with average surface roughnesses of approximately $0.025 \mu \mathrm{m}(1 \mu \mathrm{in})$ to $0.15 \mu \mathrm{m} \quad(6 \mu \mathrm{in})$. In fact, improved surface conditioning is one of the more important advances in present day magnetic tape production methods. The equation which is used for determining the loss of signal amplitude due to head-to-media spacing is

$\operatorname{Loss}(\mathrm{dB})=\frac{54.5 \mathrm{~d}}{\lambda}$

where $d=$ head-to-media distance.

$$
\lambda=\text { signal wavelengt } h
$$

Note that the spacing loss is most severe for short wavelength, high density recordings.

All of the preceding material explains why magnetic media which is int ended for high density performance should have surface coatings which are as thin and as smooth as possible, and also have as high a coercivity 
$\mathrm{H}_{c}$ as the writing transducers can accommodate. However, the demands for an output signal level from media which can function with conventional equipment, requires that the surface not be made so thin as to produce a very weak output signal. This is particularly true at low signal frequencies, since low density recording usually produces a deeper penetration into the media surface and therefore requires a thicker surface coating than the high density, short wavelength recordings.

\subsection{Magnetic Media Particles [40],[41]}

(a) The surface coatings of almost all computer magnetic tapes produced today consist of ferromagnetic needle-shaped or "acicular" iron oxide particles which are dispersed in an organic, typically, polyurethane binder. The combination of these acicular particles and the binder results in a deep brown colored mixture with a paint-like consistency. The processes which are required to achieve, maintain, and store this mixture are considered to be proprietary by each manufacturer. The mixture, in its wet state, is coated uniformly by one of several processes on to a clear, flexible plastic tape base web material usually made of polyethylene terephthalate (mylar). If the binder and oxide have been mixed in the proper proportions (the oxide particles usually constitute a volume fraction of about $35 \%$ to $45 \%$ of the coating), then the tape remains flexible and pliable af ter the coating dries.

While the coating is still wet, the tape is subjected to a strong magnetic field which physically turns and orients the needle-shaped particles so that their long axis is parallel to the longitudinal direction of the mylar (typically, $0.6 \mathrm{~m}(2 \mathrm{ft})$ ) base web. The strength of the magnetic field, the viscosity of the coating mix, and how well the particles are dispersed in the binder material will affect the quality of the final particle orientation. If these factors are not carefully controlled, the oxide particles will "clump" or "rope" together and produce a rough tape both physically and magnetically after the coating is dried. Drying is performed with the aid of heat and a substantial flow of air. It is important to note that when dried, the acicular oxide particles are permanently fixed in position and thereafter do not move physically when subjected to the strong writing head magnetic fields. At this point, the tape web is slit into $12.7 \mathrm{~mm}(0.5 \mathrm{in})$ widths and $730 \mathrm{~m}(2400 \mathrm{ft})$ lengths; these constitute the computer magnetic tapes. The slitting process must be very carefully controlled.

Many present day computer tapes are also backcoated with a thin, polyurethane binder which contains black, nonmagnetic, conductive, carbon based particles. This backcoating reduces static buildup in the tape, provides improved frictional characteristics, and reduces the production and the effects of tape debris.

(b) The ferromagnetic material used in almost all computer storage media is magnetically hard, gamma ferric oxide $\left(\gamma-\mathrm{Fe}_{2}{ }_{3}\right)$ particles. Typically, these particles are acicular (needle-like) in shape with a $6: 1$ length-to-width ratio, the length being about $0.50 \mu \mathrm{m}(20 \mu \mathrm{in})$. The size and volume of these particles causes them to behave as "single domain" 
particles. Single-domain particles cannot maintain domain walls and their state or direction of magnetization is altered by magnetic vector rotation rather than by the domain wall displacements that take place in large bulk magnetic materials.

High coercivities $\mathrm{H}_{\mathrm{c}}$ are achieved in single-domain particles by making the particles acicular. Acicularity produces what is known as shape anisotropy and gives each particle two (easy) directions of minimum free energy from which it is difficult to reverse the direction of magnetization. This is useful for magnetic tape since there is one direction of linear tape movement and therefore the particles can be oriented with their long axis parallel to this direction. In the case of magnetic disks, it is more difficult to use acicular particles because they cannot be oriented easily (although disks are available in oriented form). In this case, $y-\mathrm{Fe}_{2} \mathrm{O}_{3}$ particles which display cubic crystalline anisotropy with six directions of easy magnetization can be used since they do not have to be physically oriented in the disk coatings. In addition, these cubic $\gamma-\mathrm{Fe}_{2}{ }^{0} 3$ particles can be doped or epitaxied with a small quantity of cobalt in order to raise their coer civity to a higher value.

Thin films of nickel-cobalt are of ten electrodeposited onto rigid disk surfaces. These are excellent for high densities because of their thinness and also because these thin films are able to produce higher intensities of magnetization than a $\gamma-\mathrm{Fe}_{2} \mathrm{O}_{3}$ particle coating of equivalent thickness. That is, they can produce high bit densities with fairly strong signal amplitudes. Unfortunately, thin metal films have not succeeded as coatings on flexible magnetic tape media because they do not adhere well to the mylar plastic base substrates and tend to rub off on the contacting recording and reproducing heads.

(c) There are magnetic particle types other than gamma ferric oxide which are available for use in computer tapes [40][41]. However, as noted, at the present time $\gamma-\mathrm{Fe}_{2} \mathrm{O}_{3}$ particles are overwhelmingly prevalent in the computer magnetic media field. This is due to their historical position, low cost, good operational characteristics, and the general field and production experience with these particles.

New particle research and development work is aimed at producing smaller, well-configured particles which have high coercivities and high intensities of magnetization in thin layers. When achieved, these parameters allow for lower noise tapes, higher bit density capabilities, higher signal levels, and smoother tapes which result in good head-to-tape contact and less wear.

Since most computer tape systems have been designed to accommodate $\gamma-\mathrm{Fe}_{2}{ }_{3} 3$ tapes, then much of the potential of new particles and media cannot be realized if they are required to operate within the constraints of these older conventional systems. This situation can be corrected through the development of systems which are specifically designed to operate with the new media. Unfortunately, multi-system approacines can create interchangeability and compatibility problems unless industry standards are developed. 
There are usually trade-of fs which must be made for each new particle. For example, chromium dioxide $\mathrm{CrO}_{2}$ particles have many good characteristics including orientability and dispersability, excellent shape, size, and surface qualities which are controllable, and high values of magnetization and coercivities in thin layers. In addition, $\mathrm{CrO}_{2}$ tapes display high bit density and signal level characteristics. However, $\mathrm{CrO}_{2}$ particle costs are high, they tend to be more abrasive than $\gamma-\mathrm{Fe}_{2} 0_{3}$, and they have a very low Curie temperature which can lead to the loss of stored data in hot environments. Considerable effort has been applied towards solving the $\mathrm{CrO}_{2}$ abrasivity (head wear) problem; in one instance, through the development of smaller, homogeneous particles.

There are $y-\mathrm{Fe}_{2} \mathrm{O}_{3}$ particles whose characteristics are improved through the chemical application of additional elements such as cobalt $\mathrm{Co}$. Co is either directly doped in to the structure of the $\gamma-\mathrm{Fe}_{2} \mathrm{O}_{3}$ (Co substituted) or cobalt ions are diffused as an epitaxial layer (Co adsorbed) onto its surface. Cobalt processing makes it possible to achieve a large range of coercivities; in fact, a $5 \%$ doping of Co makes it possible to achieve a coercivity of $80,000 \mathrm{~A} / \mathrm{m}(1000 \mathrm{Oe})$. However, cobalt substitution produces particles with a greater $\mathrm{H}_{\mathrm{c}}$ temperature sensitivity and increased print-through in stored tapes.

A class of non-oxide, metal particles such as $\mathrm{Fe}$ and $\mathrm{Fe}-\mathrm{Co}$ have been developed. They have very high magnetizations, high coercivities and remain ferromagnetic even when they are extremely small. A direct substitution of $\mathrm{Fe}$ for $\gamma-\mathrm{Fe}_{2}{ }_{3}$ yields a $+12 \mathrm{~dB}$ signal increase [41]. Unfortunately, these metal particles will rust or burn when exposed to air and therefore require special protective coatings which are under development at the present time. 


\section{Symbols and Abbreviations}

\begin{tabular}{|c|c|}
\hline A & amperes \\
\hline ADP & automatic data processing \\
\hline ANSI & A merican National Standards Institute \\
\hline ASTM & American Society for Testing and Materials \\
\hline bpi & bits per inch \\
\hline bpm m & bits per millimeter \\
\hline BOT & beginning of tape (marker) \\
\hline cgs & centimeter gram second \\
\hline $\mathrm{C}$ & Celsius \\
\hline Co & cobalt \\
\hline $\mathrm{CO}_{2}$ & carbon dioxide \\
\hline CPI & characters per inch \\
\hline CP U & central processing unit \\
\hline $\mathrm{CrO}_{2}$ & chromium dioxide \\
\hline d & distance \\
\hline $\mathrm{dB}$ & decibels \\
\hline emu & electromagnetic units \\
\hline & equation \\
\hline EOT & end-of-tape (marker) \\
\hline fig. & figure \\
\hline $\mathrm{ft}$ & feet \\
\hline $\mathrm{ft}^{2}$ & square feet \\
\hline $\mathrm{ft}^{3}$ & cubic feet \\
\hline $\mathrm{F}$ & Fahrenheit \\
\hline $\mathrm{Fe}$ & iron \\
\hline FSS & Federal Supply Service \\
\hline GCR & group coded recording \\
\hline GSA & General Services Administration \\
\hline Gy & gray (absorbed dose) \\
\hline $\mathrm{H}$ & magnetic field intensity \\
\hline $\mathrm{H}_{\mathrm{c}}$ & coercivity \\
\hline $\mathrm{H}_{\mathrm{d}}$ & demagnetization field \\
\hline HEP A & high efficiency particulate air filter \\
\hline $\mathrm{Hz}$ & hertz \\
\hline in & inch \\
\hline in 2 & square inches \\
\hline in 3 & cubic inches \\
\hline I & intensity of magnetization \\
\hline ICST & Institute for Computer Sciences and Technology \\
\hline IEEE & Institute of Electrical and Electronics Engineers \\
\hline $\mathrm{kg}$ & kilogram \\
\hline
\end{tabular}




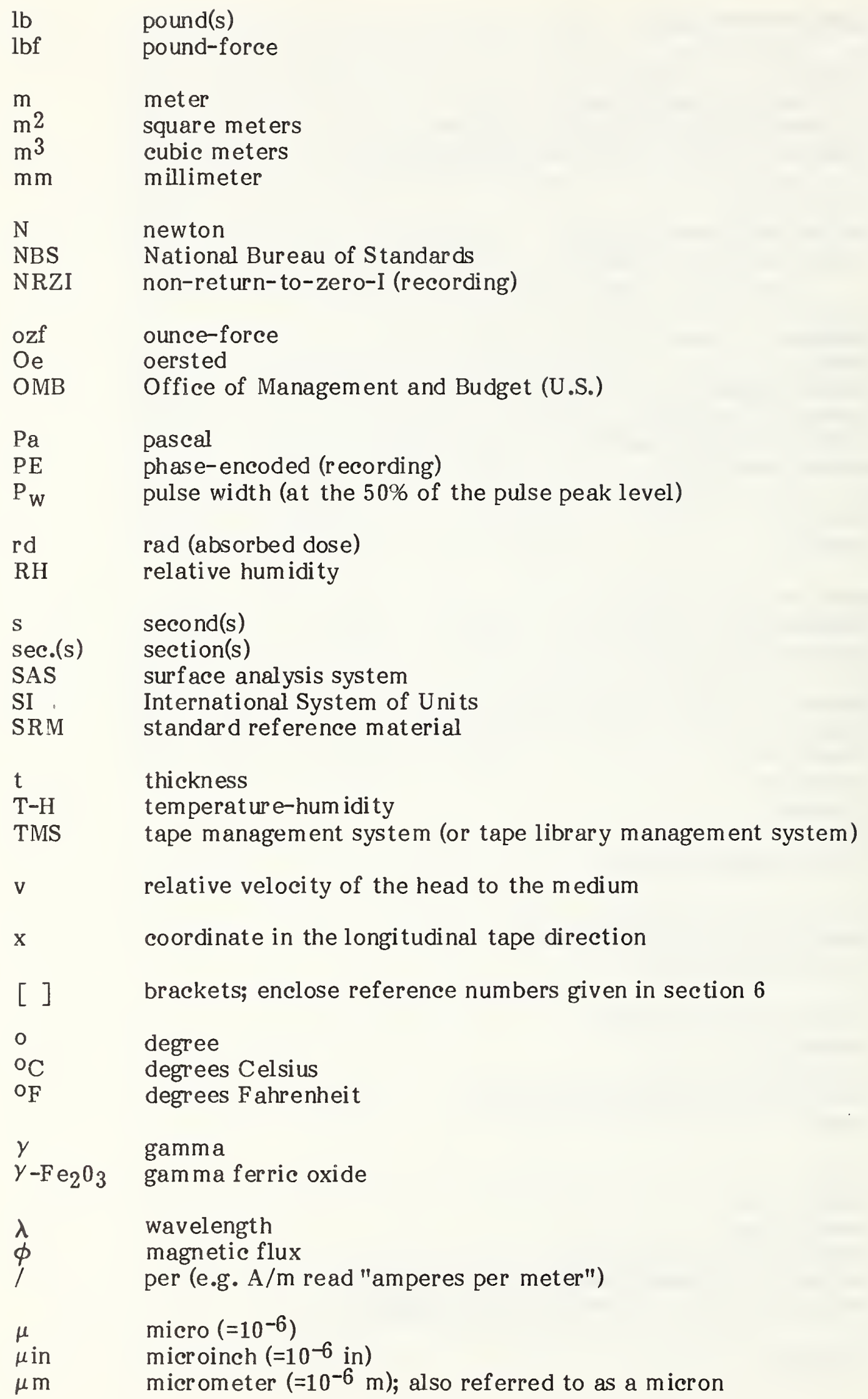


NBS.114A (REV. 2-8C)

\begin{tabular}{|c|c|c|c|}
\hline $\begin{array}{l}\text { U.s. DEPT. OF COMM. } \\
\text { BIBLIOGRAPHIC DATA } \\
\text { SHEET (See instructions) }\end{array}$ & $\begin{array}{l}\text { 1. PUBLICATION OR } \\
\text { REPORT NO. } \\
\text { NBS SP } 500-101\end{array}$ & 2. Performing Organ. Report No. & $\begin{array}{c}\text { 3. Publication Date } \\
\text { June } 1983\end{array}$ \\
\hline 4. TITLE AND SUBTITLE & COMPUTER SO & & \\
\hline
\end{tabular}

5. $\operatorname{AUTHOR}(S)$

Sidney B. Geller

6. PERFORMING ORGANIZATION (If joint or other than NBS. see instructions)

NATIONAL BUREAU OF STANDARDS

DEPARTMENT OF COMMERCE

WASHINGTON, D.C. 20234

9. SPONSORING ORGANIZATION NAME AND COMPLETE ADDRESS (Street, City. State, ZIP)

Same as item 6 .

10. SUPPLEMENTARY NOTES

Library of Congress Catalog Card Number: 83-600536

[] Document describes a computer program; SF-185, FIPS Software Summary, is attached.

11. ABSTRACT (A 200-word or less factual summary of most significant information. If document includes a significant bibliography or literature survey. mention it here)

This Special Publication deals with the physical/chemical preservation of computer magnetic storage media-principally computer magnetic tapes--and their stored data, through the application of proper care and handling methods under various conditions. It emphasizes the media handling methods and environmental conditions which should be instituted during the course of controllable day-to-day and long-term archival storage activities. It also considers measures which can be initiated during media transit and in the aftermath of catastrophic or uncontrollable events. However, the publication does not address the problem of computer storage system security from the viewpoint of data theft or intentional data modification.

12. KEY WORDS (Six to twelve entries; alphabetical order; capitalize only proper names; and separate key words by semicolons) Archival storage; care and handling; certification/evaluation; computer tape, magnetic; clean rooms; envirommental effects; flexible disks; inagnetic fields, effeets of; rehabilitation, computer tape; scheduled maintenance, computer tape; storage media, computer magnetic; tape

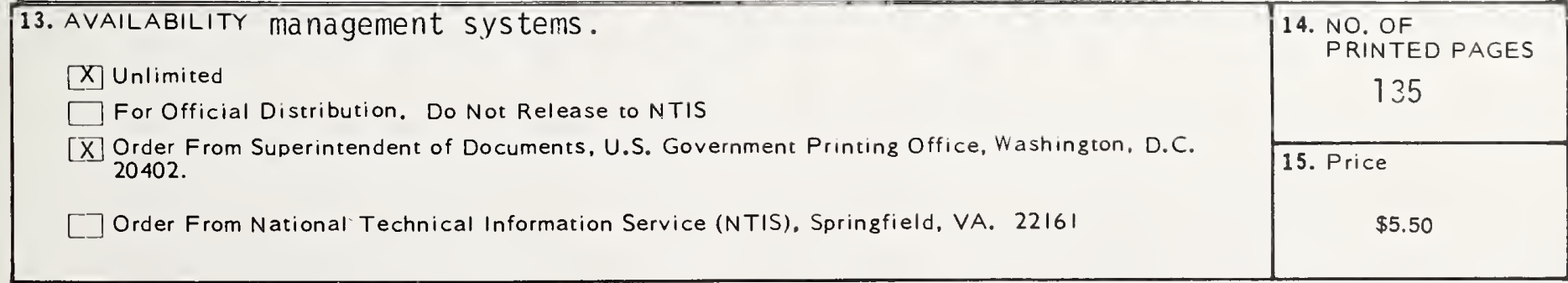






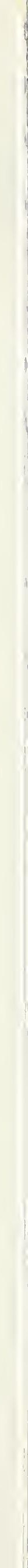




\section{NBS TECHNICAL PUBLICATIONS}

\section{PERIODICALS}

JOURNAL OF RESEARCH-The Journal of Research of the National Bureau of Standards reports NBS research and development in those disciplines of the physical and engineering sciences in which the Bureau is active. These include physics, chemistry, engineering, mathematics, and computer sciences. Papers cover a broad range of subjects, with major emphasis on measurement methodology and the basic technology underlying standardization. Also included from time to time are survey articles on topics closely related to the Bureau's technical and scientific programs. As a special service to subscribers each issue contains complete citations to all recent Bureau publications in both NBS and nonNBS media. Issued six times a year. Annual subscription: domestic $\$ 18$; foreign $\$ 22.50$. Single copy. $\$ 5.50$ domestic; $\$ 6.90$ foreign.

\section{NONPERIODICALS}

Monographs-Major contributions to the technical literature on various subjects related to the Bureau's scientific and technical activities.

Handbooks-Recommended codes of engineering and industrial practice (including safety codes) developed in cosperation with interested industries, professional organizations, and regulatory bodies.

Special Publications-Include proceedings of conferences sponsored by NBS, NBS annual reports, and other special publications appropriate to this grouping such as wall charts, pocket cards, and bibliographies.

Applied Mathematics Series-Mathematical tables, manuals, and studies of special interest to physicists, engineers, chemists, biologists, mathematicians, computer programmers, and others engaged in scientific and technical work

National Standard Reference Data Series-Provides quantitative data on the physical and chemical properties of materials, compiled from the world's literature and critically evaluated. Developed under a worldwide program coordinated by NBS under the authority of the National Standard Data Act (Public Law 90-396).

NOTE: The principal publication outlet for the foregoing data is the Journal of Physical and Chemical Reference Data (JPCRD) published quarterly for NBS by the American Chemical Society (ACS) and the American Institute of Physics (AIP). Subscriptions, reprints, and supplements available from ACS, IIS5 Sixteenth St. NW, Washington, DC 20056.
Building Science Series-Disseminates technical ir'ormatıon developed at the Bureau on building materials, coinponents, systems, and whole structures. The series presents research results, test methods, and performance criteria related to the structural and environmental functions and the durability and safety characteristics of building elements and systems.

Technical Notes-Studies or reports which are complete in themselves but restrictive in their treatment of a subject. Analogous to monographs but not so comprehensive in scope or definitive in treatment of the subject area. Often serve as a vehicle for final reports of work performed at NBS under the sponsorship of other government agencies

Voluntary Product Standards-Developed under procedures published by the Department of Commerce in Part 10. Title 15, of the Code of Federal Regulations. The standards establish nationally recognized requirements for products, and provide all concerned interests with a basis for common understanding of the characteristics of the products. NBS administers this program as a supplement to the activities of the private sector standardizing organizations.

Consumer Information Series-Practical information, based on NBS research and experience, covering areas of interest to the consumer. Easily understandable language and illustrations provide useful background knowledge for shopping in today"s technological marketplace.

Order the above NBS publications from: Superintendent of Docu. ments, Government Printing Office, Washington, DC 20402.

Order the following NBS publications-FIPS and NBSIR's-from the National Technical Information Service, Springfield. VA 22161

Federal Information Processing Standards Publications (FIPS PUB)-Publications in this series collectively constitute the Federal Information Processing Standards Register. The Register serves as the official source of information in the Federal Government regarding standards issued by NBS pursuant to the Federal Property and Administrative Services Act of 1949 as amended, Public Law 89-306 (79 Stat. 1127), and as implemented by. Executive Order 11717 (38 FR 12315, dated May 11, 1973) and Part 6 of Title 15 CFR (Code of Federal Regulations)

NBS Interagency Reports (NBSIR)-A special series of interim or final reports on work performed by NBS for outside sponsors (both government and non-government). In general, initial distribution is handled by the sponsor; public distribution is by the National Technical Information Service, Springfield, VA 22161. in paper copy or microfiche form. 
U.S. Department of Commerce

National Bureau of Standards

Washington, D.C. 20234

Official Business

Penalty for Private Use $\$ 300$

POSTAGE AND FEES PAID US DEPARTMENT OF COMMERCE COM-215

THIRO CLASS BULK RATE 